\title{
Constructing totally positive piecewise Chebyshevian B-spline bases
}

\author{
Marie-Laurence Mazure \\ Université Grenoble Alpes, Laboratoire Jean Kuntzmann, CNRS, UMR 5224, \\ BP 53, F-38041 Grenoble 9, France \\ mazure@imag.fr
}

\begin{abstract}
We consider piecewise Chebyshevian splines, in the sense of splines with pieces taken from any different five-dimensional Extended Chebyshev spaces, and with connection matrices at the knots. In this large context we establish necessary and sufficient conditions for the existence of totally positive refinable B-spline bases. These conditions are applied in many important special cases, e.g., symmetric cardinal geometrically continuous quartic B-spline, parametrically continuous mixed L-splines. The great variety of the illustrations provided proves the richness of this class of splines and its interest for design.
\end{abstract}

Keywords: Total positivity, B-spline bases, Bernstein-type bases, Extended Chebyshev spaces, generalised derivatives, blossoms, shape parameters, geometric design

AMS subject classification: 65D17,65D07

\section{Introduction}

The usefulness of Extended Chebyshev spaces and Chebyshevian splines (i.e., splines with all pieces taken from the same Extended Chebyshev space and with parametric continuity conditions at the knots) for design is beyond question. It is due to the great variety of shape parameters this class of spaces provides. The relevance of considering spaces of splines whose pieces are taken from different Extended Chebyshev spaces no longer needs to be demonstrated either. Such spaces are expected to enhance the shape possibilities, but to be of interest for design they must ensure the presence of totally positive refinable B-spline bases. To increase the chances to obtain such bases, a wise precaution consists in allowing the continuity between consecutive pieces to be controlled by connection matrices. In short, we then refer to such splines as piecewise Chebyshevian splines.

In the case where the section-spaces are defined by means of systems of weight functions, the continuity conditions can be expressed via the associated generalised derivatives rather than via the ordinary derivatives. If so, the total positivity of the corresponding connection matrices is sufficient to guarantee the presence of a B-spline basis. This was first proved by P.J. Barry in [4], where he extended similar results previously obtained by several authors for geometrically continuous polynomial splines $[17,15,16]$.

An alternative approach of piecewise Chebyshevian splines, based on blossoming, was developed with a view to replacing sufficient conditions by necessary and sufficient conditions. The existence of blossoms was proved to be the exact property that piecewise Chebyshevian spline spaces should satisfy. This existence is indeed equivalent to the existence of refinable B-spline bases $[29,33]$. Moreover, the properties of blossoms permit the development of the classical design algorithms 
and guarantee the total positivity of these bases [29, 28, 32, 33]. The avantages of the blossoming approach concerning the amplitude of the possible shape effects was indisputably demonstrated with four-dimensional section-spaces in [30]. Unfortunately, in higher dimensions, Barry's total positivity assumption on the connection matrices has long remained the only practical way to guarantee existence of blossoms. Nevertheless, to apply it, a non-trivial preliminary step consists in determining systems of weight functions associated to the given section-spaces. In [35], a remarkably simple theoretical description of the complete class of all piecewise Chebyshevian splines which can be used for design - that is, which possess blossoms -, was eventually achieved. It can be summarised as follows: on each interval, take any possible system of weight functions, and at each knot, connect the left and right selected generalised derivatives via identity matrices. This provides us with the complete class in question. A piecewise Chebyshevian spline space being given, with connection conditions expressed in terms of the ordinary derivatives, the previous simple recipe naturally results in a constructive way to answer the question: can we design with this space or not? This is made possible by the fact that we know how to obtain all possible generalised derivatives which can be associated with a given Extended Chebyshev space on a closed bounded interval [34]. Being able to answer the previous question is not only important for geometric design, but it also gives access to spline interpolation [39], approximation by Schoenberg-type operators [38], multiresolution analysis [24], along with applications in various domains, e.g., isogeometric analysis.

The purpose of the present article is to derive from the theoretical results mentioned above practical necessary and sufficient conditions for the most general piecewise Chebyshevian spline spaces with five-dimensional section-spaces to be good for design (see the exact definition in Section 2 ). Our main motivation is the fact that dimension five permits many more interesting shapes than dimension four (see, for instance [9]). The conditions in question are established in Section 4, Theorem 4.1, which is our main result. Already briefly mentioned above, the crucial results on which Theorem 4.1 relies are reminded in a more precise way in Section 2. They are completed in Section 3 by technical preliminaries on a recursive construction of Bernstein-type bases in connection with generalised differentiation. Practical aspects are addressed in Section 5, e.g., how to guarantee symmetry preservation. In the remaining sections, we examine several interesting situations to illustrate Theorem 4.1. In practice we have to drastically reduce the number of shape parameters on which the spline space depends (coming from the section-spaces and/or from the connection matrices) and Theorem 4.1 enables us to find the exact region where to choose the parameters. We successively consider: the symmetric cardinal case in Section 6, with special emphasis on symmetric (geometrically continuous) cardinal quartic B-splines; parametrically continuous mixed L-splines in Section 7, the effect of locally introducing connection matrices of certain kinds in Section 8, and finally piecewise Müntz spaces in Section 9. Concluding comments are presented in Section 10.

\section{Background}

This section aims at recalling the crucial results which the present work relies on. Our overview will be as brief as possible. For further acquaintance with Extended Chebyshev spaces, see, for instance, $[45,43,31,32,28,34]$, with Chebyshevian splines, see [45, 43, 5, 8], with piecewise Chebyshevian splines, see [4, 29, 28, 40, 41, 33, 35].

\subsection{Extended Chebyshev spaces}

We start with a concise presentation of Extended Chebyshev spaces and two main associated tools: bases of the Bernstein-type and generalised derivatives. 


\subsubsection{Extended Chebyshev spaces, Bernstein bases, and blossoms}

Let $I$ be a real interval with a non-empty interior and let $\mathbb{E} \subset C^{n}(I)$ be an $(n+1)$-dimensional linear space. Then, $\mathbb{E}$ is a $W$-space on $I$ if the Wronskian of any basis of $\mathbb{E}$ does not vanish on $I$. It is an Extented Chebyshev space (for short, EC-space) on $I$ if any non-zero function $F \in \mathbb{E}$ vanishes at most $n$ times in $I$, multiplicities included up to $(n+1)$. An EC-space on $I$ is a $\mathrm{W}$-space on $I$, the converse is not true.

As classical examples, all null spaces of linear differential operators of order $(n+1)$ with constant coefficients of which the characteristic polynomials have only real roots are $(n+1)$-dimensional ECspace on $I=\mathbb{R}$. It is well known that when the characteristic polynomial has at least one non-real root, then the null space - which is a W-space on $\mathbb{R}$ - is not an EC-space on the whole of $\mathbb{R}$, but only on sufficiently small intervals (at least on any interval of length less than $\pi / a$, where $a$ denotes the greatest imaginary part of all non-real roots of the characteristic polynomial).

Blossoms and Bernstein type bases are intrinsically connected with EC-spaces. These links, essential for geometric design, are recalled below.

Theorem 2.1. [28], [31] Given any $(n+1)$-dimensional space $\mathbb{E} \subset C^{n}(I)$, supposed to be a $W$-space on $I$ and to contain constants, the following properties are equivalent:

(i) blossoms exist in $\mathbb{E}$;

(ii) $\mathbb{E}$ possesses a Bernstein basis relative to any $(c, d) \in I^{2}, c<d$;

(iii) the space $D \mathbb{E}:=\left\{D F:=F^{\prime} \mid F \in \mathbb{E}\right\}$ is an (n-dimensional) EC-space on I;

(iv) the space $D \mathbb{E}$ possesses a Bernstein-like basis relative to any $(c, d) \in I^{2}, c<d$;

Furthermore, if (i) is satisfied, then it is possible to develop all the classical geometric design algorithms in $\mathbb{E}$, and the Bernstein basis relative to any $(c, d)$ is the optimal normalised totally positive basis in the restriction of $\mathbb{E}$ to $[c, d]$.

Let us now briefly explain the various tools involved in Theorem 2.1. Given any $c, d \in I, c<d$, we say that a sequence $\left(V_{0}, \ldots, V_{n}\right)$ of functions in $C^{n}(I)$ is a Bernstein-like basis relative to $(c, d)$ if, for each $k=0, \ldots, n, V_{k}$ vanishes exactly $k$ times at $c$, and exactly $(n-k)$ times at $d$, and is positive on $] c, d[$. Such a Bernstein-like basis is said to be a Bernstein basis relative to $(c, d)$ if it is normalised, i.e., if it satisfies $\sum_{i=0}^{n} V_{i}=\mathbb{I}$, where $\mathbb{I}$ denotes the constant function $\mathbb{I}(x):=1$ for all $x \in I$.

When they exist, blossoms are geometrical objects defined by means of intersections of osculating flats. For the exact definition, see [43], [31], [32]. When $\mathbb{E}$ possesses blossoms, each function $F \in \mathbb{E}$ is associated with a function $f: I^{n} \rightarrow \mathbb{R}$ (its blossom) satisfying three fundamental properties: it is symmetric on $I^{n}$, it gives $F$ by restriction to the diagonal of $I^{n}$, and it is pseudoaffine in each variable, [31], [32], which extends the classical affinity in each variable of polynomial blossoms [?]. The latter three properties permit the development of all the classical geometric design algorithms, in particular of de Casteljau-type evaluation algorithms. The fact that these algorithms are corner cutting on the concerned intervals guarantees the total positivity of the corresponding Bernstein bases on these intervals (concerning total positivity and shape preservation, see [28], and also [18], [?]). This brief reminder highly justifies the following terminology:

Definition 2.2. Given a $\mathrm{W}$-space $\mathbb{E}$ on $I$, assumed to contain constants, we say that $\mathbb{E}$ is good for design when blossoms exist in $\mathbb{E}$.

As a consequence of Theorem 2.1, a W-space on $I$ which is good for design is an EC-space on $I$. 
Remark 2.3. Suppose that $\mathbb{E}$ is good for design. One key point in the proof of Theorem 2.1 is the fundamental link between Bernstein bases in $\mathbb{E}$ and Bernstein-like bases in $D \mathbb{E}$ [31]. We describe it below, for it will be crucial in the reminder of the present work. Given any $a, b \in I, a<b$, let $\left(B_{0}, \ldots, B_{n}\right)$ be the Bernstein basis relative to $(a, b)$ in the EC-space. Consider the functions

$$
B_{i}^{\star}:=\sum_{k=i}^{n} B_{i}=\mathbb{I}-\sum_{k=0}^{i-1} B_{i}, \quad i=0, \ldots, n,
$$

so that, in particular,

$$
B_{0}^{\star}=\mathbb{I}, \quad B_{n}^{\star}=B_{n}, \quad B_{i}=B_{i}^{\star}-B_{i+1}^{\star} \text { for } i=0, \ldots, n,
$$

with the convention that $B_{n+1}^{\star}=0$. It is easily seen that the sequence $\left(V_{0}, \ldots, V_{n-1}\right)$ defined by

$$
V_{i}:=D B_{i+1}^{\star}=\sum_{k=i+1}^{n} D B_{k}=-\sum_{k=0}^{i} D B_{k}, \quad i=0, \ldots, n-1,
$$

is a Bernstein-like basis relative to $(a, b)$ in the space $D \mathbb{E}$. Moreover, expansions in that basis can easily be derived from expansions in the Bernstein basis. Indeed, given any real numbers $\alpha_{0}, \ldots, \alpha_{n}$

$$
U:=\sum_{i=0}^{n} \alpha_{i} B_{i} \quad \Longrightarrow \quad D U:=\sum_{i=0}^{n-1}\left(\alpha_{i+1}-\alpha_{i}\right) V_{i} .
$$

\subsubsection{EC-spaces and generalised derivatives}

A sequence $\left(w_{0}, \ldots, w_{n}\right)$ is said to be a system of weight functions on $I$ if, for $i=0, \ldots, n$, the function $w_{i}$ is positive and $C^{n-i}$ on the interval $I$. With such a system it is classical to associate a sequence $L_{0}, \ldots, L_{n}$ of linear differential operators on $C^{n}(I)$ obtained by alternating division by a weight function and ordinary differentiation as follows:

$$
L_{0} F:=\frac{F}{w_{0}}, \quad L_{i} F:=\frac{D L_{i-1} F}{w_{i}}, i=1, \ldots, n .
$$

For each $i \leqslant n$, the operator $L_{i}$ is of order $i$. These operators $L_{0}, \ldots, L_{n}$ are often referred to as the generalised derivatives associated with the system $\left(w_{0}, \ldots, w_{n}\right)$. The space $\mathbb{E}$ composed of all functions $F \in C^{n}(I)$ for which the last generalised derivative $L_{n} F$ is constant on $I$ is an $(n+1)$-dimensional EC-space on $I$. This well-known fact is due to the class of all EC-spaces on a given interval being closed under integration and multiplication by sufficiently differentiable non-vanishing functions. The $(n+1)$-dimensional EC-space $\mathbb{E}$ in question is called the EC-space associated with the system $\left(w_{0}, \ldots, w_{n}\right)$. We denote it as $\mathbb{E}=E C\left(w_{0}, \ldots, w_{n}\right)$. Take $I=\mathbb{R}$, and $w_{i}=\mathbb{I}$ for $i=0, \ldots, n$. Then, the associated generalised derivatives $L_{0}, \ldots, L_{n}$ are simply the ordinary derivatives $D^{0}=\operatorname{Id}, D, D^{2}, \ldots, D^{n}$, and the space $E C\left(w_{0}, \ldots, w_{n}\right)$ is thus the polynomial space $\mathcal{P}_{n}$.

Observe that, without any requirement on the interval $I$, it is not at all guaranteed that a given $(n+1)$-dimensional EC-space on $I$ can be associated with a system of weight functions on $I$.

Theorem 2.4. [43], [31] Suppose that the interval I is closed and bounded. Then, if $\mathbb{E}$ is an $(n+1)$-dimensional EC-space on I, there exist systems $\left(w_{0}, \ldots, w_{n}\right)$ of weight functions on I such that $\mathbb{E}=E C\left(w_{0}, \ldots, w_{n}\right)$.

As a consequence of Definition 2.2 and of Theorems 2.4 and 2.1, we can state: 
Corollary 2.5. Suppose that the interval I is closed and bounded. Then, an $(n+1)$-dimensional $W$-space $\mathbb{E}$ on $I$ is good for design if and only if there exist systems $\left(w_{1}, \ldots, w_{n}\right)$ of weight functions on $I$ such that $\mathbb{E}=E C\left(\mathbb{I}, w_{1}, \ldots, w_{n}\right)$.

The construction of all systems of weight functions associated with a given EC-space on a closed bounded interval was achieved in [34]. They are obtained by iterating the theorem below, based on Theorem 2.1, and on the properties of blossoms. This is one of the key results on which the present work relies.

Theorem 2.6. [34] Let $\mathbb{E}$ be an $(n+1)$-dimensional EC-space on a given closed bounded interval $[a, b], a<b$. Then, given any $w_{0} \in \mathbb{E}$, the following properties are equivalent

(i) all coordinates of $w_{0}$ in a given Bernstein-like basis relative to $(a, b)$ are positive;

(ii) $w_{0}$ is positive on $[a, b]$ and, setting $L_{0} V:=V / w_{0}$ for all functions $V$ defined on $I$, the $W$-space $L_{0} \mathbb{E}$ is good for design (i.e., $D L_{0} \mathbb{E}$ is an $E C$-space on $I$ ).

Remark 2.7. Theorem 2.6 describes the transformation of any EC-space $\mathbb{E}$ on $[a, b], a<b$, into an ECspace good for design on $[a, b]$ of the same dimension, out of division by a convenient positive function. We recall here how this transformation operates on bases. Let $\left(V_{0}, \ldots, V_{n}\right)$ be any Bernstein-like basis relative to $(a, b)$ in $\mathbb{E}$. Let $w_{0} \in \mathbb{E}$ be defined by $w_{0}:=\sum_{i=0} \alpha_{i} V_{i}$, where $\alpha_{0}, \ldots, \alpha_{n}$. are positive. Division by $w_{0}$ yields

$$
\mathbb{I}=\sum_{i=0}^{n} B_{i}, \quad \text { where } B_{i}:=\frac{\alpha_{i} V_{i}}{w_{0}} \text { for } i=0, \ldots, n .
$$

Clearly, the functions $B_{0}, \ldots, B_{n}$ form the Bernstein basis relative to $(a, b)$ in the space $L_{0} \mathbb{E}$.

Remark 2.8. Given any non-trivial real interval $J$, let $L_{0}, \ldots, L_{n}$ be the generalised derivatives on $C^{n}(J)$ associated with a given system $\left(w_{0}, \ldots, w_{n}\right)$ of weight functions on $J$. The recursive definition (4) yields a lower triangular matrix functional matrix $\Gamma_{p}\left(w_{0}, \ldots, w_{n} ;.\right)$ defined on the interval $J$ such that

$$
\left(F^{\prime}(x), \ldots, F^{(n)}(x)\right)^{T}=\Gamma_{n}\left(w_{0}, \ldots, w_{n} ; x\right)\left(L_{0} F(x), \ldots, L_{n} F(x)\right)^{T}, \quad 1 \leqslant p \leqslant n, \quad x \in J .
$$

The formal expression of this matrix is independent of $J$, and we will therefore keep a standard notation on all intervals. For details, see [37]. For instance, the diagonal of $\Gamma_{n}\left(w_{0}, \ldots, w_{n} ;.\right)$ is equal to $\left(w_{0}, w_{0} w_{1}, \ldots, \prod_{i=0}^{n} w_{i}\right)$, while its first column is $\left(w_{0}, w_{0}{ }^{\prime}, \ldots, w_{0}{ }^{(n)}\right)^{T}$.

\subsection{Piecewise Chebyshevian splines}

Throughout the article we shall consider a fixed bi-infinite sequence of knots $t_{k}, k \in \mathbb{Z}$, with $t_{k}<t_{k+1}$ for all $k$. Each $t_{k}$ is allocated a multiplicity $m_{k}$, with

$$
0 \leqslant m_{k} \leqslant n \quad \text { for all } k \in \mathbb{Z}, \quad \sum_{i \leqslant 0} m_{i}=\sum_{i \geqslant 0} m_{i}=+\infty .
$$

The extended knot-vector $\mathbb{K}:=\left(t_{k}{ }^{\left[m_{k}\right]}\right)_{k \in \mathbb{Z}}$ formed by the knots repeated with their multiplicities can then be written as a bi-infinite sequence

$$
\mathbb{K}=\left(\xi_{\ell}\right)_{\ell \in \mathbb{Z}}, \quad \text { with } \xi_{\ell} \leqslant \xi_{\ell+1} \text { and } \xi_{\ell}<\xi_{\ell+n} \text { for all } \ell \in \mathbb{Z}
$$

To define a spline space we additionally consider: 
- a bi-infinite sequence of section spaces $\mathbb{E}_{k}, k \in \mathbb{Z}$ : each $\mathbb{E}_{k}$ is an $(n+1)$-dimensional EC-space good for design on $\left[t_{k}, t_{k+1}\right]$ (i.e., it contains constants and the $n$-dimensional space $D \mathbb{E}_{k}$ is an EC-space on $\left.\left[t_{k}, t_{k+1}\right]\right)$;

- a bi-infinite sequence of connection matrices $M_{k}, k \in \mathbb{Z}$ : each $M_{k}$ is a lower triangular matrix of order $\left(n-m_{k}\right)$ with positive diagonal entries.

A piecewise Chebyshevian spline based on these data is a continuous function $S:] \inf _{k} t_{k}, \sup _{k} t_{k}[\rightarrow \mathbb{R}$ meeting the following requirements:

1- for each $k \in \mathbb{Z}$, there exists a function $F_{k} \in \mathbb{E}_{k}$ which coincides with $S$ on the interval $\left[t_{k}, t_{k+1}\right]$; 2- $S$ satisfies the connection conditions:

$$
\left(S^{\prime}\left(t_{k}^{+}\right), \ldots, S^{\left(n-m_{k}\right)}\left(t_{k}^{+}\right)\right)^{T}=M_{k}\left(S^{\prime}\left(t_{k}^{-}\right) \ldots, S^{\left(n-m_{k}\right)}\left(t_{k}^{-}\right)\right)^{T}, \quad k \in \mathbb{Z} .
$$

Subsequently we denote by $\mathbb{S}$ the set all such splines. This space contains constants. Moreover, splines in $\mathbb{S}$ are geometrically continuous in the sense of continuity of the left/right Frenet frames of order three and of the first two left/right curvatures at each knot, which is usually referred to as $F^{3}$ continuity. A short spline version of Theorem 2.1 can be stated as follows. A more complete one can be found in [35].

Theorem 2.9. [29], [33] For the piecewise Chebyshevian spline space $\mathbb{S}$ defined above, the following three properties are equivalent:

(i) blossoms exist in $\mathbb{S}$;

(ii) $\mathbb{S}$ possesses a B-spline basis, and so does any spline space obtained from $\mathbb{S}$ by knot insertion;

(iii) the space $D \mathbb{S}$ possesses B-spline-like bases, and so does any spline space obtained from DS by knot insertion.

Furthermore, if (i) is satisfied, then it is possible to develop all the classical geometric design algorithms for splines in $\mathbb{S}$, and its B-spline basis is totally positive.

When blossoms exist in $\mathbb{S}$, they are as earlier defined in a geometrical way by means of intersections of osculating flats, and they satisfy the same three fundamental properties. However, the major difference is that, in the spline framework, the natural domain of definition of blossoms is a restricted set of $n$-tuples $\left.\mathbb{A}_{n}(\mathbb{K}) \subset\right] \inf _{k} t_{k}, \sup _{k} t_{k}\left[{ }^{n}\right.$, said to be admissible with respect to the knotvector $\mathbb{K}$. An $n$-tuple $\left.\left(x_{1}, \ldots, x_{n}\right) \in\right] \inf _{k} t_{k}, \sup _{k} t_{k}\left[{ }^{n}\right.$ is admissible if, for each $k \in \mathbb{Z}$ such that $\min \left(x_{1}, \ldots, x_{n}\right)<t_{k}<\max \left(x_{1}, \ldots, x_{n}\right)$, the knot $t_{k}$ appears at least $m_{k}$ times in the sequence $\left(x_{1}, \ldots, x_{n}\right)$.

A sequence $N_{\ell} \in \mathbb{S}, \ell \in \mathbb{Z}$, is a $B$-spline basis if it meets the usual requirements below:

- support property: for each $\ell \in \mathbb{Z}, N_{\ell}$ has support $\left[\xi_{\ell}, \xi_{\ell+n+1}\right]$;

- positivity property: for each $\ell \in \mathbb{Z}, N_{\ell}$ is positive on $] \xi_{\ell}, \xi_{\ell+n+1}[$;

- normalisation property: $\sum_{\ell \in \mathbb{Z}} N_{\ell}(x)=1$ for all $\left.x \in\right] \inf _{k} t_{k}, \sup _{k} t_{k}[$

- endpoint property: for each $\ell \in \mathbb{Z}, N_{\ell}$ vanishes exactly $(n-s+1)$ times at $\xi_{\ell}$ and exactly $\left(n-s^{\prime}+1\right)$ at $\xi_{\ell+n+1}$, where $s:=\sharp\left\{j \geqslant \ell \mid \xi_{j}=\xi_{\ell}\right\}$ and $s^{\prime}:=\sharp\left\{j \leqslant \ell+n+1 \mid \xi_{j}=\xi_{\ell+n+1}\right\}$.

Splines in the space $D \mathbb{S}$ may fail to be continuous at some knots. However, a B-spline-like basis in $D \mathbb{S}$ is defined in a similar way simply replacing $n$ by $n-1$ and omitting the normalisation property. Theorem 2.9 justifies the following definition.

Definition 2.10. The piecewise Chebyshevian spline space $\mathbb{S}$ is said to be good for design when blossoms exist in it. 
For each $k$, select any system $\left(w_{1}^{k}, \ldots, w_{n}^{k}\right)$ of weight functions on $\left[t_{k}, t_{k+1}\right]$ so that $D \mathbb{E}_{k}=$ $E C\left(w_{1}^{k}, \ldots, w_{n}^{k}\right)$. Let $L_{0}^{k}, L_{1}^{k}, \ldots, L_{n}^{k}$ denote the generalised derivatives on $C^{n}\left(\left[t_{k}, t_{k+1}\right]\right)$ associated with the system $\left(\mathbb{1}_{k}, w_{1}^{k}, \ldots, w_{n}^{k}\right)$ via (4). Then, the connection conditions (8) can be expressed by means of these generalised derivatives as follows:

$$
\left(L_{1}^{k} S\left(t_{k}^{+}\right), \ldots, L_{n-m_{k}}^{k} S\left(t_{k}^{+}\right)\right)^{T}=\bar{M}_{k}\left(L_{1}^{k-1} S\left(t_{k}^{-}\right), \ldots, L_{n-m_{k}}^{k-1} S\left(t_{k}^{+}\right)\right)^{T}, \quad k \in \mathbb{Z},
$$

where, due to $(5)$, the matrix $\bar{M}_{k}$ (lower triangular matrix of order $\left(n-m_{k}\right)$ with positive diagonal entries), is given by:

$$
\bar{M}_{k}:=\Gamma_{n-m_{k}}\left(w_{1}^{k}, \ldots, w_{n-m_{k}}^{k} ; t_{k}^{+}\right)^{-1} M_{k} \Gamma_{n-m_{k}}\left(w_{1}^{k-1}, \ldots, w_{n-m_{k}}^{k-1} ; t_{k}^{-}\right)
$$

We conclude this section with the second key result on which the present work relies. Obtained by iterating the spline version of Theorem 2.6, it provides us with a beautiful and simple theoretical characterisation of existence of blossoms.

Theorem 2.11. [35] The following two statements are equivalent:

(i) the $P W$-spline space $\mathbb{S}$ is good for design;

(ii) among all bi-infinite sequences $\left(w_{1}^{k}, \ldots, w_{n}^{k}\right), k \in \mathbb{Z}$, - where, for each $k,\left(w_{1}^{k}, \ldots, w_{n}^{k}\right)$ is a system of weight functions on $\left[t_{k}, t_{k+1}\right]$ ensuring that $D \mathbb{E}_{k}=E C\left(w_{1}^{k}, \ldots, w_{n}^{k}\right)$, - one can find one such that, for each $k \in \mathbb{Z}$, the matrix $\bar{R}_{k}$ involved in the connection conditions (9) is the identity matrix of order $\left(n-m_{k}\right)$.

Remark 2.12. According to (10), condition (ii) in Theorem 2.11 is equivalent to the existence of a biinfinite sequence of systems of weight functions $\left(w_{1}^{k}, \ldots, w_{n}^{k}\right), k \in \mathbb{Z}$, so that $D \mathbb{E}_{k}=E C\left(w_{1}^{k}, \ldots, w_{n}^{k}\right)$ and

$$
\Gamma_{n-m_{k}}\left(w_{1}^{k}, \ldots, w_{n-m_{k}}^{k} ; t_{k}^{+}\right)=M_{k} \Gamma_{n-m_{k}}\left(w_{1}^{k-1}, \ldots, w_{n-m_{k}}^{k-1} ; t_{k}^{-}\right), \quad k \in \mathbb{Z}
$$

\section{Technical preliminaries}

It the present section we develop technical results necessary to establish our main result in next section. They concern the search for systems of weight functions associated with a given EC-space (here of dimension four) as iterated application of Theorem 2.6.

Throughout the section, the interval $[a, b], a<b$, is given and $\mathbb{E} \subset C^{4}([a, b])$ is a five-dimensional EC-space which is good for design on $[a, b]$. In other words $\mathbb{E}$ contains constants, and the space $D \mathbb{E}$ is a (four-dimensional) EC-space on $[a, b]$.

Let us start with a system $\left(w_{1}, w_{2}, w_{3}, w_{4}\right)$ of weight functions on $[a, b]$ such that

$$
\mathbb{E}=E C\left(\mathbb{I}, w_{1}, w_{2}, w_{3}, w_{4}\right), \quad \text { i.e., } D \mathbb{E}=\left(w_{1}, w_{2}, w_{3}, w_{4}\right) .
$$

Let $L_{0}, \ldots, L_{4}$ denote the generalised derivatives associated with $\left(\mathbb{I}, w_{1}, w_{2}, w_{3}, w_{4}\right)$. Being obtained by iteration of Theorem 2.6 , the system $\left(w_{1}, w_{2}, w_{3}, w_{4}\right)$ is completely specified by a total amount of ten positive coefficients, namely the positive coordinates of the four functions $w_{1}, w_{2}, w_{3}, w_{4}$ in given Bernstein-like bases successively of $D \mathbb{E}$, of $D L_{1} \mathbb{E}$, of $D L_{2} \mathbb{E}$, and finally of $D L_{3} \mathbb{E}$. As explained in Remarks 2.3 and 2.7, all such bases can be deduced from one selected Bernstein-like basis of the space $D \mathbb{E}$. The arguments we are developing below are valid in any dimension. However, of course, the greater the dimension is, the more difficult the computations are.

Here, we are actually interested only in $\left(w_{1}, w_{2}, w_{3}\right)$, and more precisely in the fact that, according to $(5)$,

$$
\left(F^{\prime}(x), F^{\prime \prime}(x), F^{\prime \prime \prime}(x)\right)^{T}=\Gamma\left(w_{1}, w_{2}, w_{3} ; x\right)\left(L_{1} F(x), L_{2} F(x), L_{3} F(x)\right)^{T}, \quad x \in[a, b],
$$


for all $F \in \mathbb{E}$, with

$$
\Gamma\left(w_{1}, w_{2}, w_{3} ; \cdot\right)=\left[\begin{array}{lll}
w_{1} & 0 & 0 \\
w_{1}^{\prime} & w_{1} w_{2} & 0 \\
w_{1}^{\prime \prime} & 2 w_{1}^{\prime} w_{2}+w_{1} w_{2}{ }^{\prime} & w_{1} w_{2} w_{3} .
\end{array}\right] .
$$

We will give more details on the construction of $\left(w_{1}, w_{2}, w_{3}\right)$, so as to achieve an explicit expression of $\Gamma\left(w_{1}, w_{2}, w_{3} ; a\right)$ and $\Gamma\left(w_{1}, w_{2}, w_{3} ; b\right)$ in terms of the positive coefficients mentioned above.

Since $D \mathbb{E}$ is an EC-space on $[a, b]$, we can define four functions $V_{i}, i=0, \ldots, 3$, as the unique solutions in $D \mathbb{E}$ of the following four Hermite interpolation problems:

$$
\begin{aligned}
& V_{0}(a)=1, \quad V_{0}(b)=V_{0}{ }^{\prime}(b)=V_{0}{ }^{\prime \prime}(b)=0, \\
& V_{1}(a)=0, V_{1}{ }^{\prime}(a)=1, \quad V_{1}(b)=V_{1}{ }^{\prime}(b)=0, \\
& V_{2}(a)=V_{2}{ }^{\prime}(a)=0, \quad V_{2}(b)=0, V_{2}^{\prime}(b)=-1, \\
& V_{3}(a)=V_{3}^{\prime}(a)=V_{3}^{\prime \prime}(a)=0, \quad V_{3}(a)=1 \text {. }
\end{aligned}
$$

Clearly, $\left(V_{0}, V_{1}, V_{2}, V_{3}\right)$ is a Bernstein-like basis of $D \mathbb{E}$ relative to $(a, b)$. From now on we will omit "relative to $(a, b)$ " since we will only consider Bernstein and Bernstein-like bases relative to $(a, b)$.

Applying Theorem 2.6, we know that the positive function $w_{1}$ can be expanded in the Bernsteinlike basis $\left(V_{0}, V_{1}, V_{2}, V_{3}\right)$ as

$$
w_{1}=\sum_{i=0}^{3} \alpha_{i} V_{i}, \quad \text { with positive } \alpha_{0}, \alpha_{1}, \alpha_{2}, \alpha_{3} .
$$

According to Theorem 2.6, the four-dimensional EC-space $L_{1} \mathbb{E}$ is good for design on $[a, b]$ and from Remark 2.7, its Bernstein basis $\left(\bar{B}_{0}, \bar{B}_{1}, \bar{B}_{2}, \bar{B}_{3}\right)$ is obtained as follows

$$
\bar{B}_{i}:=\frac{\alpha_{i} V_{i}}{w_{1}} \text { for } i=0, \ldots, 3 .
$$

The three-dimensional space $D L_{1} \mathbb{E}$ is thus an EC-space on $[a, b]$. As a Bernstein-like basis $\left(\bar{V}_{0}, \bar{V}_{1}, \bar{V}_{2}\right)$ of $D L_{1} \mathbb{E}$, we can take (see Remark 2.3)

$$
\bar{V}_{i}:=D\left(\sum_{j=i+1}^{3} \bar{B}_{j}\right)=-D\left(\sum_{j=0}^{i} \bar{B}_{j}\right), \quad i=0,1,2 .
$$

Applying again Theorem 2.6, the positive function $w_{2} \in D L_{1} \mathbb{E}$ can be expanded as

$$
w_{2}=\sum_{i=0}^{2} \beta_{i} \bar{V}_{i}, \quad \text { with positive } \beta_{0}, \beta_{1}, \beta_{2} .
$$

Remark 2.7 shows that the sequence $\left(\overline{\bar{B}}_{0}, \overline{\bar{B}}_{1}, \overline{\bar{B}}_{2}\right)$ defined by

$$
\overline{\bar{B}}_{i}:=\frac{\beta_{i} \bar{V}_{i}}{w_{2}} \text { for } i=0, \ldots, 2 .
$$

is the Bernstein basis of the three-dimensional EC-space $L_{2} \mathbb{E}$ which is good for design on $[a, b]$. The last step we need to describe is as follows:

$$
w_{3}=\sum_{i=0}^{1} \gamma_{i} \overline{\bar{V}}_{i}, \quad \text { with positive } \gamma_{0}, \gamma_{1},
$$


where $\left(\overline{\bar{V}}_{0}, \bar{V}_{1}\right)$ denotes the following Bernstein-like basis of the two-dimensional space $D L_{2} \mathbb{E}$, known to be an EC-space on $[a, b]$

$$
\overline{\bar{V}}_{0}:=D \overline{\bar{B}}_{1}+D \overline{\bar{B}}_{2}=-D \overline{\bar{B}}_{0}, \quad \overline{\bar{V}}_{1}:=D \overline{\bar{B}}_{2}=-D \overline{\bar{B}}_{0}-D \overline{\bar{B}}_{1} .
$$

Via (13), (16), (18), the matricial function $\Gamma\left(w_{1}, w_{2}, w_{3} ; \cdot\right)$ thus depends on the nine positive coefficients $\alpha_{0}, \alpha_{1}, \alpha_{2}, \alpha_{3}, \beta_{0}, \beta_{1}, \beta_{2}, \gamma_{0}, \gamma_{1}$. Let us have a look at what happens at the endpoints of the interval $[a, b]$. With this aim in mind, we must introduce some notations concerning the derivatives of higher order of the Bernstein-like basis (12). To be consistent with the next subsection, below the upper signs,+- indicate that we are dealing with $a=a^{+}$and $b=b^{-}$, respectively.

$$
\begin{array}{lll}
A^{+}:=-V_{0}{ }^{\prime}(a), & B^{+}:=V_{0}^{\prime \prime}(a), \quad A^{-}:=V_{3}{ }^{\prime}(b), & B^{-}:=V_{3}^{\prime \prime}(b), \\
D^{+}:=V_{1}^{\prime \prime}(a), & C^{-}:=V_{1}^{\prime \prime}(b), \quad C^{+}:=V_{2}^{\prime \prime}(a), & D^{-}:=V_{2}^{\prime \prime}(b) .
\end{array}
$$

Remark 3.1. Due to (12), the positivity of the two functions $V_{1}, V_{2}$ on $] a, b[$ ensures that

$$
C^{+}>0, \quad C^{-}>0 .
$$

All other coefficients introduced in (20) are a priori of any non-strict sign.

Remark 3.2. Assume that the space $D \mathbb{E}$ is closed under reflection through $(a+b) / 2$. Then,

$$
V_{0}(x)=V_{3}(a+b-x), \quad V_{1}(x)=V_{2}(a+b-x), \quad \text { for all } x \in[a, b] .
$$

This yields

$$
A^{+}=A^{-}, \quad B^{+}=B^{-}, \quad C^{+}=C^{-},
$$

which justifies the choice for the notations introduced in 20 .

On account of how the Bernstein-like basis $\left(V_{0}, \ldots, V_{3}\right)$ is defined in (12), and of the notations introduced in (20), we have

$$
\begin{aligned}
& w_{1}(a)=\alpha_{0} V_{0}(a)=\alpha_{0}, \\
& w_{1}{ }^{\prime}(a)=\alpha_{0} V_{0}^{\prime}(a)+\alpha_{1} V_{1}{ }^{\prime}(a)=-A^{+} \alpha_{0}+\alpha_{1}, \\
& w_{1}^{\prime \prime}(a)=\alpha_{0} V_{0}^{\prime \prime}(a)+\alpha_{1} V_{1}^{\prime \prime}(a)+\alpha_{2} V_{2}^{\prime \prime}(a)=B^{+} \alpha_{0}+D^{+} \alpha_{1}+C^{+} \alpha_{2},
\end{aligned}
$$

and

$$
\begin{aligned}
& w_{1}(b)=\alpha_{3} V_{3}(b)=\alpha_{3}, \\
& w_{1}^{\prime}(b)=\alpha_{2} V_{2}^{\prime}(b)+\alpha_{3} V_{3}^{\prime}(b)=-\alpha_{2}+A^{-} \alpha_{3} \\
& w_{1}^{\prime \prime}(b)=\alpha_{1} V_{1}^{\prime \prime}(b)+\alpha_{2} V_{2}^{\prime \prime}(b)+\alpha_{3} V_{3}^{\prime \prime}(b)=C^{-} \alpha_{1}+D^{-} \alpha_{2}+B^{-} \alpha_{3}
\end{aligned}
$$

The values at $a, b$ of the function $w_{2}$ and of its first derivative similarly involve those of the Bernsteinlike basis $\left(\bar{V}_{0}, \bar{V}_{1}, \bar{V}_{2}\right)$ which can themselves be calculated from those of $\left(V_{0}, \ldots, V_{3}\right)$ via $(15)$ and (22), (23). This gives

$$
\begin{aligned}
& w_{2}(a)=\beta_{0} \bar{V}_{0}(a)=\beta_{0} \frac{\alpha_{1}}{\alpha_{0}}, \quad w_{2}(b)=\beta_{2} \bar{V}_{2}(b)=\beta_{2} \frac{\alpha_{2}}{\alpha_{3}} \\
& w_{2}^{\prime}(a)=\beta_{0} \bar{V}_{0}^{\prime}(a)+\beta_{1} \bar{V}_{1}^{\prime}(a)=-\beta_{0} \frac{\alpha_{1}}{\alpha_{0}}\left[2 \frac{\alpha_{1}}{\alpha_{0}}-C^{+} \frac{\alpha_{2}}{\alpha_{1}}-\left(2 A^{+}+D^{+}\right)\right]+\beta_{1} C^{+} \frac{\alpha_{2}}{\alpha_{0}}, \\
& w_{2}^{\prime}(b)=\beta_{2} \bar{V}_{2}^{\prime}(b)+\beta_{3} \bar{V}_{3}^{\prime}(b)=\beta_{2} \frac{\alpha_{2}}{\alpha_{3}}\left[2 \frac{\alpha_{2}}{\alpha_{3}}-C^{-} \frac{\alpha_{1}}{\alpha_{2}}-\left(2 A^{-}+D^{-}\right)\right]-\beta_{1} C^{-} \frac{\alpha_{1}}{\alpha_{3}}
\end{aligned}
$$

Finally, from (18) and (19) we can also derive that

$$
w_{3}(a)=\gamma_{0} \frac{\beta_{1}}{\beta_{0}} \frac{\alpha_{2}}{\alpha_{1}} C^{+}, \quad w_{3}(b)=\gamma_{1} \frac{\beta_{1}}{\beta_{2}} \frac{\alpha_{1}}{\alpha_{2}} C^{-}
$$


Gathering all previous relations leads to:

$$
\begin{aligned}
\Gamma\left(w_{1}, w_{2}, w_{3} ; a\right) & =\left[\begin{array}{lll}
\alpha_{0} & 0 & 0 \\
-A^{+} \alpha_{0}+\alpha_{1} & \alpha_{1} \beta_{0} & 0 \\
B^{+} \alpha_{0}+D^{+} \alpha_{1}+C^{+} \alpha_{2} & \beta_{0}\left(C^{+} \alpha_{2}+D^{+} \alpha_{1}\right)+C^{+} \beta_{1} \alpha_{2} & C^{+} \alpha_{2} \beta_{1} \gamma_{0}
\end{array}\right], \\
\Gamma\left(w_{1}, w_{2}, w_{3} ; b\right) & =\left[\begin{array}{lll}
\alpha_{3} & 0 & 0 \\
-\alpha_{2}+A^{-} \alpha_{3} & \alpha_{2} \beta_{2} & 0 \\
C^{-} \alpha_{1}+D^{-} \alpha_{2}+B^{-} \alpha_{3} & \beta_{2}\left(-C^{-} \alpha_{1}-D^{-} \alpha_{2}\right)-C^{-} \beta_{1} \alpha_{1} & C^{-} \alpha_{1} \beta_{1} \gamma_{1}
\end{array}\right] .
\end{aligned}
$$

\section{Quartic-like piecewise Chebyshevian splines}

In this section, we consider the space $\mathbb{S}$ of piecewise Chebyshevian splines described in Subsection 2.3 , with now $n=4$ and simple knots, the connection matrices being given by

$$
M_{k}=\left[\begin{array}{lll}
a_{k} & 0 & 0 \\
b_{k} & c_{k} & 0 \\
d_{k} & e_{k} & f_{k}
\end{array}\right], \quad \text { with } a_{k}, c_{k}, f_{k}>0 \text { for all } k \in \mathbb{Z} .
$$

For each $k \in \mathbb{Z}$, in the EC-space $D \mathbb{E}_{k}$ we denote by $\left(V_{0}^{k}, V_{1}^{k}, V_{2}^{k}, V_{3}^{k}\right)$ the Bernstein-like basis defined by conditions $(12)$, in which $(a, b)$ is replaced by $\left(t_{k}, t_{k+1}\right)$. Moreover, for each $k \in \mathbb{Z}$, we use the lower index $k$ for all the notations similar to (20) concerning the knot $t_{k}$, that is,

$$
\begin{array}{llll}
A_{k}^{-}:=V_{3}^{k-1^{\prime}}\left(t_{k}\right), & B_{k}^{-}:=V_{3}^{k-1^{\prime \prime}}\left(t_{k}\right), \quad A_{k}^{+}:=-V_{0}^{k^{\prime}}\left(t_{k}\right), \quad B_{k}^{+}:=V_{0}^{k^{\prime \prime}}\left(t_{k}\right), \\
C_{k}^{-}:=V_{1}^{k-1^{\prime \prime}}\left(t_{k}\right), \quad D_{k}^{-}:=V_{2}^{k-1^{\prime \prime}}\left(t_{k}\right), \quad D_{k}^{+}:=V_{1}^{k^{\prime \prime}}\left(t_{k}\right), \quad C_{k}^{+}:=V_{2}^{k^{\prime \prime}}\left(t_{k}\right) .
\end{array}
$$

With these notations, we can state our main result in Theorem 4.1 below as a consequence of our theoretical characterisation given in Theorem 2.11.

Theorem 4.1. The space $\mathbb{S}$ of all piecewise Chebyshevian splines with connection matrices (27) described above is good for design if and only if the connection matrices satisfy the following conditions:

$$
\widetilde{B}_{k}>0, \quad \widetilde{D}_{k}>0, \quad C_{k}^{+} C_{k+1}^{-} \widetilde{B}_{k} \widetilde{B}_{k+1} f_{k+1}<\widetilde{D}_{k}\left(\widetilde{B}_{k+1} \widetilde{E}_{k+1}-c_{k+1} \widetilde{D}_{k+1}\right) \quad \text { for all } k \in \mathbb{Z},
$$

where, for each $k \in \mathbb{Z}$, the numbers $\widetilde{B}_{k}, \widetilde{E}_{k}, \widetilde{D}_{k}$ are defined by

$$
\begin{aligned}
& \widetilde{B}_{k}:=b_{k}+a_{k} A_{k}^{+}+c_{k} A_{k}^{-}, \\
& \widetilde{E}_{k}:=e_{k}-c_{k} D_{k}^{+}-f_{k} D_{k}^{-}, \\
& \widetilde{D}_{k}:=d_{k}+e_{k} A_{k}^{-}-b_{k} D_{k}^{+}+a_{k}\left(-B_{k}^{+}-D_{k}^{+} A_{k}^{+}\right)-c_{k} D_{k}^{+} A_{k}^{-}+f_{k} B_{k}^{-} .
\end{aligned}
$$

Proof. We know that the spline space $\mathbb{S}$ is good for design if and only if it possible to find, for each $k \in \mathbb{Z}$, a system $\left(w_{1}^{k}, w_{2}^{k}, w_{3}^{k}, w_{4}^{k}\right)$ of weight functions on $\left[t_{k}, t_{k+1}\right]$, so as to ensure the following two properties for each $k \in \mathbb{Z}$ :

$$
\begin{aligned}
& D \mathbb{E}_{k}=E C\left(w_{1}^{k}, w_{2}^{k}, w_{3}^{k}, w_{4}^{k}\right), \\
& \Gamma\left(w_{1}^{k}, w_{2}^{k}, w_{3}^{k} ; t_{k}\right)=M_{k} \cdot \Gamma\left(w_{1}^{k-1}, w_{2}^{k-1}, w_{3}^{k-1} ; t_{k}\right) .
\end{aligned}
$$

The analysis carried out in the previous section shows that the search for a system $\left(w_{1}^{k}, w_{2}^{k}, w_{3}^{k}, w_{4}^{k}\right)$ of weight functions on $\left[t_{k}, t_{k+1}\right]$ ensuring (31) is equivalent to the search for ten positive coefficients $\alpha_{0}^{k}, \alpha_{1}^{k}, \alpha_{2}^{k}, \alpha_{3}^{k}, \beta_{0}^{k}, \beta_{1}^{k}, \beta_{2}^{k}, \gamma_{0}^{k}, \gamma_{1}^{k}, \delta_{0}^{k}$, starting from the Bernstein basis $\left(V_{0}^{k}, V_{1}^{k}, V_{2}^{k}, V_{3}^{k}\right)$ of $D \mathbb{E}_{k}$ relative to $\left(t_{k}, t_{k+1}\right)$. For any given $k$, using (26), relation (32) can be regarded as a system in the nine unknowns $\alpha_{0}^{k}, \alpha_{1}^{k}, \alpha_{2}^{k}, \alpha_{3}^{k}, \beta_{0}^{k}, \beta_{1}^{k}, \beta_{2}^{k}, \gamma_{0}^{k}, \gamma_{1}^{k}$, with the nine numbers concerning the previous interval as parameters. The proof can thus be split into two parts: 
Part 1: For a given integer $k$, can we solve "positively" (32)? That is, can we find necessary and sufficient conditions involving given nine positive parameters $\alpha_{0}^{k-1}, \alpha_{1}^{k-1}, \alpha_{2}^{k-1}, \alpha_{3}^{k-1}$, $\beta_{0}^{k-1}, \beta_{1}^{k-1}, \beta_{2}^{k-1}, \gamma_{0}^{k-1}, \gamma_{1}^{k-1}$ and the six entries of the connection matrix $M_{k}$, leading to positive $\alpha_{0}^{k}, \alpha_{1}^{k}, \alpha_{2}^{k}, \alpha_{3}^{k}, \beta_{0}^{k}, \beta_{1}^{k}, \beta_{2}, \gamma_{0}^{k}, \gamma_{1}^{k}$ via (32)?

Part 2: Can we globally solve positively all systems (32)? That is, can we make all necessary and sufficient conditions provided by Part 1 globally compatible?

The proof of Theorem 4.1 will be completed after considering these points successively in Propositions 4.2 and 4.3 below.

Let us select any $k \in \mathbb{Z}$. From (26), one can see that the equality (32) can equivalently be replaced by the six following equations:

$$
\begin{gathered}
\alpha_{0}^{k}=a_{k} \alpha_{3}^{k-1}, \\
\alpha_{1}^{k}-A_{k}^{+} \alpha_{0}^{k}=b_{k} \alpha_{3}^{k-1}+c_{k}\left(A_{k}^{-} \alpha_{3}^{k-1}-\alpha_{2}^{k-1}\right), \\
B_{k}^{+} \alpha_{0}^{k}+D_{k}^{+} \alpha_{1}^{k}+C_{k}^{+} \alpha_{2}^{k}=d_{k} \alpha_{3}^{k-1}+e_{k}\left(A_{k}^{-} \alpha_{3}^{k-1}-\alpha_{2}^{k-1}\right)+f_{k}\left(B_{k}^{-} \alpha_{3}^{k-1}+D_{k}^{-} \alpha_{2}^{k-1}+C_{k}^{-} \alpha_{1}^{k-1}\right), \\
\alpha_{1}^{k} \beta_{0}^{k}=c_{k} \alpha_{2}^{k-1} \beta_{2}^{k-1}, \\
\beta_{0}^{k}\left(C_{k}^{+} \alpha_{2}^{k}+D_{k}^{+} \alpha_{1}^{k}\right)+C_{k}^{+} \beta_{1}^{k} \alpha_{2}^{k}=e_{k} \alpha_{2}^{k-1} \beta_{2}^{k-1}+f_{k}\left[\beta_{2}^{k-1}\left(-D_{k}^{-} \alpha_{2}^{k-1}-C_{k}^{-} \alpha_{1}^{k-1}\right)-C_{k}^{-} \beta_{1}^{k-1} \alpha_{1}^{k-1}\right] . \\
C_{k}^{+} \alpha_{2}^{k} \beta_{1}^{k} \gamma_{0}^{k}=f_{k} C_{k}^{-} \alpha_{1}^{k-1} \beta_{1}^{k-1} \gamma_{1}^{k-1},
\end{gathered}
$$

Proposition 4.2 below addresses the "positive resolution" of the system formed by the latter six equations.

Proposition 4.2. An integer $k \in \mathbb{Z}$ being given, let $\alpha_{1}^{k-1}, \alpha_{2}^{k-1}, \alpha_{3}^{k-1}, \beta_{1}^{k-1}, \beta_{2}^{k-1}, \gamma_{0}^{k-1}$ be any given positive numbers. Then, there exist positive numbers $\alpha_{0}^{k}, \alpha_{1}^{k}, \alpha_{2}^{k}, \beta_{0}^{k}, \beta_{1}^{k}, \gamma_{0}^{k}$, such that all six equalities (33) to (37) hold if and only if the following two properties below are satisfied:

(1) the quantities introduced in (30) fulfill the conditions below

$$
\widetilde{B}_{k}>0, \quad \widetilde{D}_{k}>0, \quad \widetilde{B}_{k} \widetilde{E}_{k}-c_{k} \widetilde{D}_{k}>0
$$

(2) the given positive coefficients are chosen so that

$$
\begin{aligned}
& \alpha_{2}^{k-1}>\frac{C_{k}^{-} \widetilde{B}_{k} f_{k} \alpha_{1}^{k-1}}{\widetilde{B}_{k} \widetilde{E}_{k}-c_{k} \widetilde{D}_{k}}, \quad \alpha_{3}^{k-1}>\frac{\alpha_{2}^{k-1} \widetilde{E}_{k}-C_{k}^{-} f_{k} \alpha_{1}^{k-1}}{\widetilde{D}_{k}}, \\
& \beta_{2}^{k-1}>\frac{C_{k}^{-} f_{k} \alpha_{1}^{k-1} \beta_{1}^{k-1}\left[\widetilde{B}_{k} \alpha_{3}^{k-1}-c_{k} \alpha_{2}^{k-1}\right]}{\alpha_{3}^{k-1}\left[\alpha_{2}^{k-1}\left(\widetilde{B}_{k} \widetilde{E}_{k}-c_{k} \widetilde{D}_{k}\right)-C_{k}^{-} \widetilde{B}_{k} f_{k} \alpha_{1}^{k-1}\right]} .
\end{aligned}
$$

Moreover, when (39) and (40) hold, the positive numbers $\alpha_{0}^{k}, \alpha_{1}^{k}, \alpha_{2}^{k}, \beta_{0}^{k}, \beta_{1}^{k}, \gamma_{0}^{k}$ are unique.

Proof. The various arguments of the proof will constantly involve not only the positivity of the six given numbers $\alpha_{1}^{k-1}, \ldots, \gamma_{0}^{k-1}$, but also the positivity of the diagonal entries $a_{k}, c_{k}, f_{k}$ (see (27)) and of $C_{k}^{-}, C_{k}^{+}$(see (20) and (21)). The reader should keep this in mind, for we will omit mentioning it at each step. Besides, $\alpha_{0}^{k}, \alpha_{1}^{k}, \alpha_{2}^{k}$ are uniquely determined by (33), (34), (35), successively. In case $\alpha_{1}^{k}, \alpha_{2}^{k}$ are both positive, then $\beta_{0}^{k}$ and $\beta_{1}^{k}$ are uniquely determined by (36) and (37), respectively, and $\beta_{0}^{k}$ itself is positive. Finally, if $\beta_{1}^{k}$ too is positive, then $\gamma_{0}^{k}$ is uniquely determined by (38) and it 
is positive. Whence the claimed uniqueness of a possible positive solution, along with the fact that we only have to care about the positivity of $\alpha_{1}^{k}, \alpha_{2}^{k}$, and $\beta_{1}^{k}$.

Observe that, on account of (33) and (36), the three equalities (34), (35), and (37) can be replaced by

$$
\begin{gathered}
\alpha_{1}^{k}=\alpha_{3}^{k-1} \widetilde{B}_{k}-c_{k} \alpha_{2}^{k-1}, \\
C_{k}^{+} \alpha_{2}^{k}=\alpha_{3}^{k-1} \widetilde{D}_{k}-\alpha_{2}^{k-1} \widetilde{E}_{k}+C_{k}^{-} f_{k} \alpha_{1}^{k-1} . \\
C_{k}^{+} \alpha_{2}^{k} \beta_{1}^{k}=\beta_{2}^{k-1}\left(\alpha_{2}^{k-1} \widetilde{E}_{k}-C_{k}^{-} f_{k} \alpha_{1}^{k-1}\right)-C_{k}^{-} f_{k} \alpha_{1}^{k-1} \beta_{1}^{k-1}-C_{k}^{+} \alpha_{2}^{k} \beta_{0}^{k} .
\end{gathered}
$$

- Let us first assume that the numbers $\alpha_{1}^{k}, \alpha_{2}^{k}$ and $\beta_{1}^{k}$ generated by (33)-(37) are positive. From (41) we can then successively derive that

$$
\widetilde{B}_{k}>0, \quad \alpha_{3}^{k-1}>\frac{c_{k} \alpha_{2}^{k-1}}{\widetilde{B}_{k}}
$$

Similarly, (43) implies

$$
\beta_{2}^{k-1}\left(\alpha_{2}^{k-1} \widetilde{E}_{k}-C_{k}^{-} f_{k} \alpha_{1}^{k-1}\right)=C_{k}^{+} \alpha_{2}^{k} \beta_{1}^{k}+C_{k}^{-} f_{k} \alpha_{1}^{k-1} \beta_{1}^{k-1}+C_{k}^{+} \alpha_{2}^{k} \beta_{0}^{k},
$$

from which we can conclude that

$$
\alpha_{2}^{k-1} \widetilde{E}_{k}-C_{k}^{-} f_{k} \alpha_{1}^{k-1}>0 .
$$

On this account, (42) successively proves that

$$
\widetilde{D}_{k}>0, \quad \alpha_{3}^{k-1}>\frac{\alpha_{2}^{k-1} \widetilde{E}_{k}-C_{k}^{-} f_{k} \alpha_{1}^{k-1}}{\widetilde{D}_{k}} .
$$

Now, taking account of (36), (41), and (42), multiplication of both sides of the equality (43) by the positive number $\alpha_{1}^{k}$ transforms it as follows:

$$
\begin{aligned}
C_{k}^{+} \alpha_{1}^{k} \alpha_{2}^{k} \beta_{1}^{k} & =\alpha_{2}^{k-1} \beta_{2}^{k-1}\left(\alpha_{1}^{k} \widetilde{E}_{k}-C_{k}^{+} c_{k} \alpha_{2}^{k}\right)-C_{k}^{-} f_{k} \alpha_{1}^{k-1} \alpha_{1}^{k}\left(\beta_{1}^{k-1}+\beta_{2}^{k-1}\right), \\
& =\alpha_{3}^{k-1} \beta_{2}^{k-1}\left[\alpha_{2}^{k-1}\left(\widetilde{B}_{k} \widetilde{E}_{k}-c_{k} \widetilde{D}_{k}\right)-C_{k}^{-} \widetilde{B}_{k} f_{k} \alpha_{1}^{k-1}\right]-C_{k}^{-} f_{k} \alpha_{1}^{k-1} \beta_{1}^{k-1}\left[\widetilde{B}_{k} \alpha_{3}^{k-1}-c_{k} \alpha_{2}^{k-1}\right] .
\end{aligned}
$$

From (44) and from the positivity of $\widetilde{B}_{k} \alpha_{3}^{k-1}-c_{k} \alpha_{2}^{k-1}\left(=\alpha_{1}^{k}\right)$, the following two inequalities readily follow

$$
\widetilde{B}_{k} \widetilde{E}_{k}-c_{k} \widetilde{D}_{k}>0, \quad \alpha_{2}^{k-1}>\frac{C_{k}^{-} \widetilde{B}_{k} f_{k} \alpha_{1}^{k-1}}{\widetilde{B}_{k} \widetilde{E}_{k}-c_{k} \widetilde{D}_{k}} .
$$

Finally, (44) and (45) clearly prove the second line of (40).

- Conversely, we now assume that conditions (39) and (40) are fulfilled. From $\widetilde{D}_{k}$ being positive and from the second inequality in (40) we can derive that $\alpha_{2}^{k}>0$. From the quantity $\widetilde{B}_{k} \widetilde{E}_{k}-c_{k} \widetilde{D}_{k}$ being positive, the first inequality in (40) can be reformulated as

$$
\widetilde{B}_{k}\left(\alpha_{2}^{k-1} \widetilde{E}_{k}-C_{k}^{-} f_{k} \alpha_{1}^{k-1}\right)>\alpha_{2}^{k-1} c_{k} \widetilde{D}_{k} .
$$

Due to the positivity of $\widetilde{B}_{k}$ and $\widetilde{D}_{k}$, this yields

$$
\frac{\alpha_{2}^{k-1} c_{k}}{\widetilde{B}_{k}}<\frac{\alpha_{2}^{k-1} \widetilde{E}_{k}-C_{k}^{-} f_{k} \alpha_{1}^{k-1}}{\widetilde{D}_{k}} .
$$


From the second inequality in (40) and (41) we can conclude that $\alpha_{1}^{k}>0$. We can thus replace (43) by (44) in the system (33)-(37). Now, taking account of the first and last inequalities in (40), (44) proves the positivity of the product $\alpha_{1}^{k} \alpha_{2}^{k} \beta_{1}^{k}$, hence we can say that $\beta_{1}^{k}>0$.

Proposition 4.2 corresponds to Part 1 of the proof of Theorem 4.1. We are now concerned with Part 2. As a matter of fact, solving this part consists in making compatible all requirements (40) obtained when $k$ ranges over $\mathbb{Z}$. It is essential to be aware that a requirement on either $\alpha_{3}^{k-1}$ or $\beta_{2}^{k-1}$ is not a problem because none of these two coefficients is involved in the connection conditions at any knot $t_{j}, j \leqslant k-1$. By contrast, $\alpha_{2}^{k-1}$ is indeed concerned with the connection conditions at the knot $t_{k-1}$. Accordingly, only ensuring the first inequality in (40) may be problematic. This justifies the following proposition.

Proposition 4.3. The data being the same as in Proposition 4.2, we assume that conditions (39) and (40) hold. Moreover, $\mu$ is a given positive number. Then the positive numbers $\alpha_{1}^{k}, \alpha_{2}^{k}$ produced by the equations (33)-(37) satisfy

$$
\alpha_{2}^{k}>\mu \alpha_{1}^{k}
$$

if and only if we have both

$$
\mu C_{k}^{+} \widetilde{B}_{k}<\widetilde{D}_{k} \quad \text { and } \quad \alpha_{3}^{k-1}>K:=\frac{\alpha_{2}^{k-1}\left(\widetilde{E}_{k}-\mu c_{k} C_{k}^{+}\right)-C_{k}^{-} \alpha_{1}^{k-1} f_{k}}{\widetilde{D}_{k}-\mu C_{k}^{+} \widetilde{B}_{k}} .
$$

Moreover, this number $K$ satisfies

$$
K>\frac{\alpha_{2}^{k-1} \widetilde{E}_{k}-C_{k}^{-} f_{k} \alpha_{1}^{k-1}}{\widetilde{D}_{k}}
$$

Proof. Let us write condition (46) as $C_{k}^{+} \alpha_{2}^{k}>C_{k}^{+} \mu \alpha_{1}^{k}$. On account of (41) and (42) it holds if and only if

$$
\alpha_{3}^{k-1}\left(\widetilde{D}_{k}-\mu C_{k}^{+} \widetilde{B}_{k}\right)>\alpha_{2}^{k-1}\left(\widetilde{E}_{k}-\mu c_{k} C_{k}^{+}\right)-C_{k}^{-} \alpha_{1}^{k-1} f_{k} .
$$

Accordingly, in order to prove that the two conditions contained in (47) are necessary and sufficient for (46) to be satisfied, we simply have to prove that (49) implies that the quantity $\widetilde{D}_{k}-\mu C_{k}^{+} \widetilde{B}_{k}$ is positive. Now, after multiplication of both sides of (49) by the positive number $\widetilde{B}_{k}$ and reordering the various terms, we obtain the equivalent inequality

$$
\left(\widetilde{D}_{k}-\mu C_{k}^{+} \widetilde{B}_{k}\right)\left(\alpha_{3}^{k-1} \widetilde{B}_{k}-\alpha_{2}^{k-1} c_{k}\right)>\alpha_{2}^{k-1}\left(\widetilde{E}_{k} \widetilde{B}_{k}-c_{k} \widetilde{D}_{k}\right)-C_{k}^{-} f_{k} \alpha_{1}^{k-1} \widetilde{B}_{k} .
$$

Due to (41), the left-hand side is equal to $\alpha_{1}^{k}\left(\widetilde{D}_{k}-\mu C_{k}^{+} \widetilde{B}_{k}\right)$. The right-hand side is positive due to (39) and (40). Given that $\alpha_{1}^{k}>0$, we can thus say that $\widetilde{D}_{k}-\mu C_{k}^{+} \widetilde{B}_{k}>0$.

To complete the proof of Proposition 4.3, we simply mention that (48) can be deduced from the right-hand side of (50) being positive. This is left to the reader.

End of the proof of Theorem 4.1: From the previous two propositions we can draw the following two conclusions:

1- For a given integer $k$, there is no problem to solve positively the system (33)-(37) with the additional demand (46). We simply have to

- add the requirement $\mu C_{k}^{+} \widetilde{B}_{k}<\widetilde{D}_{k}$ to all conditions (39) concerning the matrix $M_{k}$;

- replace the requirement on $\alpha_{3}^{k-1}$ obtained in (40) by the stronger one $\alpha_{3}^{k-1}>L$, where $L$ is defined in (47). 
2- We recall that $\alpha_{3}^{k-1}$ is not concerned by the connection conditions at any knot preceding $t_{k}$. Accordingly, via Proposition 4.2, globally solving positively all systems (33)-(37), $k \in \mathbb{Z}$, consists in solving separately each system (33)-(37) corresponding to a given $k \in \mathbb{Z}$, with the additional requirement that

$$
\alpha_{2}^{k}>\mu_{k} \alpha_{1}^{k}, \quad \text { with } \mu_{k}:=\frac{C_{k+1}^{-} \widetilde{B}_{k+1} f_{k+1}}{\widetilde{B}_{k+1} \widetilde{E}_{k+1}-c_{k+1} \widetilde{D}_{k+1}} .
$$

Our previous comments show that this global positive resolution is possible if and only if all conditions (39) are satisfied for all $k \in \mathbb{Z}$, along with the additional ones

$$
\mu_{k} C_{k}^{+} \widetilde{B}_{k}<\widetilde{D}_{k}, \quad k \in \mathbb{Z} .
$$

These additional inequalities provide us with the rightmost condition claimed in (29) of Theorem 4.1. Apart from this, if we compare (39) with (29) of Theorem 4.1, we can observe that the positivity of all quantities $\widetilde{B}_{k} \widetilde{E}_{k}-c_{k} \widetilde{D}_{k}$ is missing in (29). Indeed, the positivity of all $\widetilde{B}_{k}$ 's, and the rightmost requirement in (29) make the two conditions " $\widetilde{D}_{k}>0$ for all $k \in \mathbb{Z}$ " and " $\widetilde{B}_{k} \widetilde{E}_{k}-c_{k} \widetilde{D}_{k}>0$ for all $k \in \mathbb{Z}$ " redundant. We can thus keep only either of them. The proof of Theorem 4.1 is complete.

To conclude this section, let us mention that the necessary and sufficient conditions Theorem 4.1 were already obtained for geometrically continuous quartic splines in [36]. Nevertheless, the question of exploiting them for obtaining interesting shapes was not at all considered there.

\section{$5 \quad$ Practical issues}

To address some practical issues in the spline space $\mathbb{S}$ it will be useful to introduce the following additional quantities:

$$
\widetilde{\Delta}_{k}:=\widetilde{B}_{k} \widetilde{E}_{k}-c_{k} \widetilde{D}_{k}, \quad \widetilde{\Gamma}_{k}:=\widetilde{D}_{k} \widetilde{\Delta}_{k+1}-C_{k}^{+} C_{k+1}^{-} \widetilde{B}_{k} \widetilde{B}_{k+1} f_{k+1}, \quad k \in \mathbb{Z} .
$$

\subsection{The B-spline basis}

Throughout the present subsection we assume that the spline space $\mathbb{S}$ is good for design. According to (29), this is equivalent to assuming that all quantities $\widetilde{B}_{k}, \widetilde{D}_{k}, \widetilde{\Gamma}_{k}, k \in \mathbb{Z}$, are positive. Moreover it implies that $\widetilde{E}_{k}, \widetilde{\Delta}_{k}$ are positive too for all $k \in \mathbb{Z}$.

Proposition 5.1. Assume that the spline space $\mathbb{S}$ is good for design. The B-spline basis $N_{j}, j \in \mathbb{Z}$, is given by

$$
N_{j}(x)=\frac{\int_{t_{j}}^{x} Q_{j}(t) d t}{\int_{t_{j}}^{t_{j+4}} Q_{j}(t) d t}-\frac{\int_{t_{j+1}}^{x} Q_{j+1}(t) d t}{\int_{t_{j+1}}^{t_{j+5}} Q_{j+1}(t) d t}, \quad j \in \mathbb{Z},
$$

where, $Q_{j}, j \in \mathbb{Z}$, is any B-spline-like basis in the space $D \mathbb{S}$. For instance, for each $j \in \mathbb{Z}$, the $B$-spline-like $Q_{j} \in D \mathbb{S}$ with support $\left[t_{j}, t_{j+4}\right]$, can be taken as 


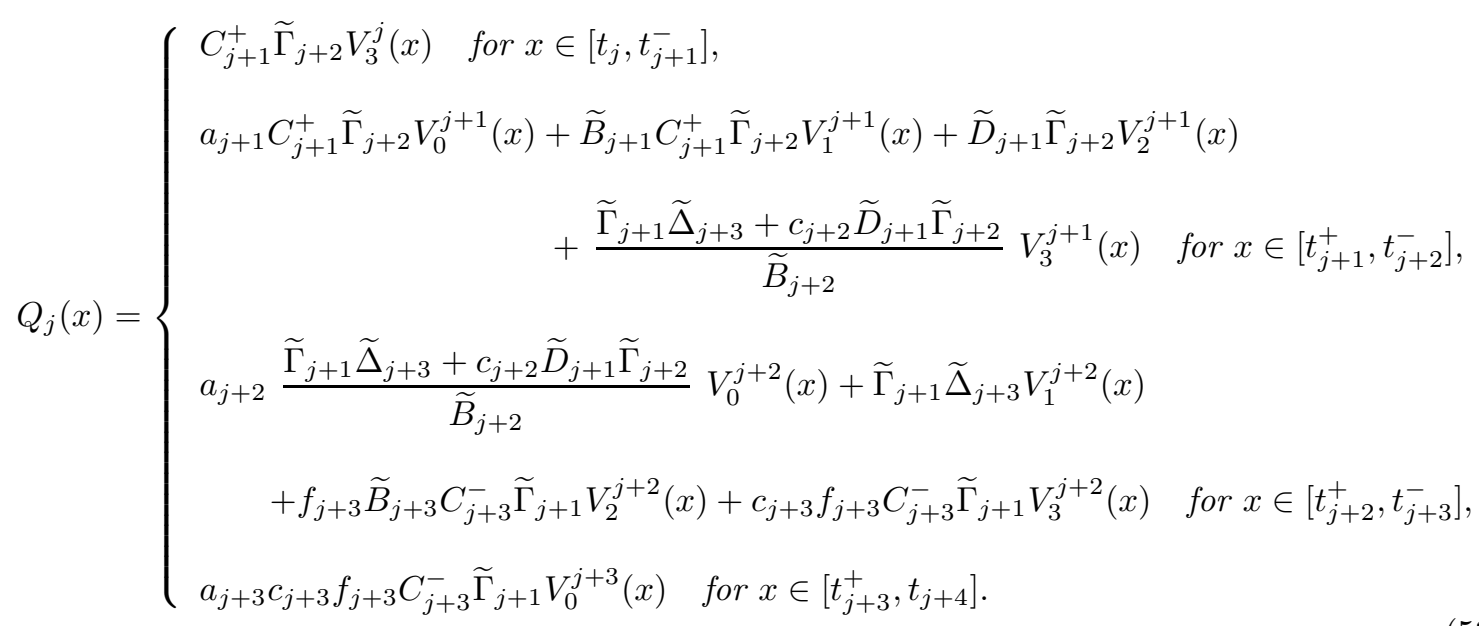

Proof. It is well known that the B-spline basis is given by $(52)$ where $Q_{j}, j \in \mathbb{Z}$, is any B-spline-like basis in the spline space. It is worthwhile insisting that the presence of connection matrices may prevent the splines in $D \mathbb{S}$ to be continuous at the knots. Therefore, without additional assumptions, a spline in $W \in D \mathbb{S}$ can only defined separately on each $\left[t_{k}^{+}, t_{k+1}^{-}\right]$. Expand it in the local Bernsteinlike bases $\left(V_{0}^{k}, V_{1}^{k}, V_{2}^{k}, V_{3}^{k}\right)$ introduced before (28) as

$$
W(x)=\sum_{i=0}^{3} \alpha_{i}^{k} V_{i}^{k}(x), \quad x \in\left[t_{k}^{+}, t_{k+1}^{-}\right], \quad k \in \mathbb{Z} .
$$

The relations between the coefficients $\alpha$ of two consecutive intervals have already been obtained in (41), (42), and (43). With the notations introduced in (30) and (51) they can be summarised as follows:

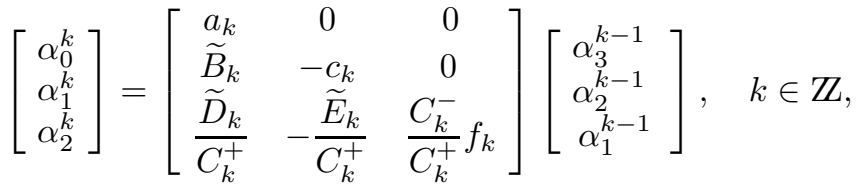

or, equivalently, as:

$$
\left[\begin{array}{c}
\alpha_{3}^{k-1} \\
\alpha_{2}^{k-1} \\
\alpha_{1}^{k-1}
\end{array}\right]=\left[\begin{array}{ccc}
\frac{1}{a_{k}} & 0 & 0 \\
\frac{\widetilde{B}_{k}}{a_{k} c_{k}} & -\frac{1}{c_{k}} & 0 \\
\frac{\Delta_{k}}{a_{k} c_{k} f_{k} C_{k}^{-}} & -\frac{\widetilde{E}_{k}}{c_{k} f_{k} C_{k}^{-}} & \frac{C_{k}^{+}}{C_{k}^{-}} \frac{1}{f_{k}}
\end{array}\right]\left[\begin{array}{c}
\alpha_{0}^{k} \\
\alpha_{1}^{k} \\
\alpha_{2}^{k}
\end{array}\right], \quad k \in \mathbb{Z} .
$$

Up to multiplication by a positive constant, the B-spline-like $M_{0}$ which is zero on any $\left[t_{k}^{+}, t_{k+1}^{-}\right]$such that $k \notin\{0,1,2,3\}$ is determined by the following conditions

$$
\alpha_{0}^{0}=\alpha_{1}^{0}=\alpha_{2}^{0}=0<\alpha_{3}^{0}, \quad \alpha_{0}^{3}>\alpha_{1}^{3}=\alpha_{2}^{3}=\alpha_{3}^{3}=0 .
$$

Requiring for instance that $\alpha_{0}^{3}=a_{3} c_{3} f_{3} C_{3}^{-}$, by application of (54) with $k=1$ and (55) with $k=3$, we obtain

$$
\alpha_{0}^{1}=a_{1} \alpha_{3}^{0}, \quad \alpha_{1}^{1}=\widetilde{B}_{1} \alpha_{3}^{0}, \quad \alpha_{2}^{1}=\frac{\widetilde{D}_{1}}{C_{1}^{+}} \alpha_{3}^{0} ; \quad \alpha_{3}^{2}=c_{3} f_{3} C_{3}^{-}, \quad \alpha_{2}^{2}=\widetilde{B}_{3} f_{3} C_{3}^{-}, \quad \alpha_{1}^{2}=\widetilde{\Delta}_{3} .
$$


Comparison between the values of $\alpha_{1}^{2}$ and $\alpha_{2}^{2}$ in the right part of (56) with those obtained from the left part of (56) via (54) yields

$$
\alpha_{3}^{0}=\frac{C_{1}^{+} \widetilde{\Gamma}_{2}}{\widetilde{\Gamma}_{1}}, \quad \alpha_{3}^{1}=\frac{\widetilde{\Delta}_{3} \widetilde{\Gamma}_{1}+c_{2} \widetilde{D}_{1} \widetilde{\Gamma}_{2}}{\widetilde{\Gamma}_{1} \widetilde{B}_{2}},
$$

which aventually yields (53).

\subsection{Symmetry issues}

Preserving the shape of the data, that is, the shape of any given control polygon, is a crucial issue in CAGD. It is therefore quite natural to try to preserve the symmetry of the data, in the sense that a control polygon presenting symmetry properties should lead to a parametric curve presenting the same symmetry properties. With this purpose in mind, assume for a while that the curve is not defined piecewisely. In that case preserving symmetry requires the use of an EC-space possessing symmetric Bernstein bases. Polynomial spaces are the most classical examples of such spaces, but more generally we can use kernels of differential operators with constant coefficients properties and even / odd characteristic polynomials. Clearly not all EC-spaces are of this type. Let us now consider spline curves. Even if we limit ourselves to symmetric spaces, the useful presence of connection matrices is likely to destroy symmetry. How can we ensure preservation of the symmetry of the data is the issue we address in the present section.

Given a real number $X_{0}$, we denote by $\vartheta: \mathbb{R} \rightarrow \mathbb{R}$ the reflection through $X_{0}$, that is

$$
\vartheta(x):=2 X_{0}-x, \quad x \in \mathbb{R} .
$$

A linear space $\mathbb{E}$ of functions defined on $[a, b], a<b$, being given, we denote by $\overleftarrow{E}$ the image of $\mathbb{E}$ through $\vartheta$, that is, the set of all functions

$$
x \mapsto F \circ \vartheta^{-1}(x), \quad x \in[\vartheta(b), \vartheta(a)], \quad F \in \mathbb{E} .
$$

Lemma 5.2. Given $t_{0}<t_{1}<t_{2}$, for $i=0,1$, let $\mathbb{E}_{i}$ be an $(n+1)$-dimensional EC-space good for design on $\left[t_{i}, t_{i+1}\right]$. Denote by $\left(\bar{t}_{0}, \bar{t}_{1}, \bar{t}_{2}\right)$ the sequence $\left(\vartheta\left(t_{2}\right), \vartheta\left(t_{1}\right), \vartheta\left(t_{0}\right)\right)$, and by $\overline{\mathbb{E}}_{0}:=\overleftarrow{\mathbb{E}}_{1}$, $\overline{\mathbb{E}}_{1}:=\overleftarrow{\mathbb{E}}_{0}$. Assume that two functions $F_{0} \in \mathbb{E}_{0}$ and $F_{1} \in \mathbb{E}_{1}$ satisfy

$$
\left(F_{1}{ }^{\prime}\left(t_{1}\right), \ldots, F_{1}{ }^{(p)}\left(t_{1}\right)\right)^{T}=M\left(F_{0}{ }^{\prime}\left(t_{1}\right), \ldots, F_{0}{ }^{(p)}\left(t_{1}\right)\right)^{T},
$$

for some integer $p \leqslant n$. Then, the functions $\bar{F}_{0}:=F_{1} \circ \vartheta, \bar{F}_{1}:=F_{0} \circ \vartheta$ satisfy

$$
\left(\bar{F}_{1}{ }^{\prime}\left(\bar{t}_{1}\right), \ldots, \bar{F}_{1}{ }^{(p)}\left(\bar{t}_{1}\right)\right)^{T}=\bar{M}\left(\bar{F}_{0}{ }^{\prime}\left(\bar{t}_{1}\right), \ldots, \bar{F}_{0}{ }^{(p)}\left(\bar{t}_{1}\right)\right)^{T},
$$

where the entry in the $i$-th row and $j$-th column of $\bar{M}$ is the $(j, i)$ minor of the matrix $M$.

Proof. It can easily be checked that

$$
\bar{M}=\Delta M^{-1} \Delta,
$$

where $\Delta$ denotes the diagonal matrix $\left(-1,(-1)^{2}, \ldots,(-1)^{p}\right)$.

Lemma 5.3. The spline space $\mathbb{S}$ is invariant under the reflection $\vartheta$ if and only the following three properties are satisfied

(i) there exists an integer $q \in \mathbb{Z}$ such that one of the two situations below hold

$$
\begin{gathered}
\text { either } X_{0}=t_{q} \quad \text { and } t_{q+k}=\vartheta\left(t_{q-k}\right) \text { for all } k \in \mathbb{Z} ; \\
\text { or } X_{0}=\frac{t_{q}+t_{q+1}}{2} \quad \text { and } t_{q+k+1}=\vartheta\left(t_{q-k}\right) \text { for all } k \in \mathbb{Z} ;
\end{gathered}
$$


(ii) for any two integers $p, j \in \mathbb{Z}$ such that $t_{p}=\vartheta\left(t_{j}\right)$ we have $\mathbb{E}_{p}=\overleftarrow{\mathbb{E}}_{j-1}$;

(iii) for any integer $j \in \mathbb{Z}$, the connection matrix $M_{p}$ at $t_{p}=\vartheta\left(t_{j}\right)$ is given

$$
M_{p}=\left[\begin{array}{lcc}
\frac{1}{a_{j}} & 0 & 0 \\
\frac{b_{j}}{a_{j} c_{j}} & \frac{1}{c_{j}} & 0 \\
\frac{b_{j} e_{j}-c_{j} d_{j}}{a_{j} c_{j} f_{j}} & \frac{e_{j}}{c_{j} f_{j}} & \frac{1}{f_{j}}
\end{array}\right] .
$$

Moreover when (i) and (ii) hold, condition (59) is equivalent to the following one:

(iv) for any integer $p, j \in \mathbb{Z}$ such that $t_{p}=\vartheta\left(t_{j}\right)$

$$
a_{p}=\frac{1}{a_{j}}, \quad c_{p}=\frac{1}{c_{j}}, \quad f_{p}=\frac{1}{f_{j}}, \quad \widetilde{B}_{p}=\frac{\widetilde{B}_{j}}{a_{j} c_{j}}, \quad \widetilde{E}_{p}=\frac{\widetilde{E}_{j}}{c_{j} f_{j}}, \quad \widetilde{D}_{p}=\frac{\widetilde{\Delta}_{j}}{a_{j} c_{j} f_{j}} .
$$

Proof. With the help of Lemma 5.2, the first part is elementary and is left to the reader. Assuming that both properties (i) and (ii) hold true, the Bernstein-like basis in $\mathbb{E}_{p}=\overleftarrow{\mathbb{E}}_{j-1}$ is obtained as

$$
V_{i}^{p}(x)=V_{3-i}^{j-1} \circ \vartheta^{-1}(x), \quad x \in\left[t_{p}, t_{p+1}\right], \quad i=0, \ldots, 3 .
$$

This yields

$$
\begin{aligned}
& A_{p}^{-}:=A_{j}^{+}, \quad B_{k}^{-}:=B_{j}^{+}, \quad A_{p}^{+}:=A_{j}^{-}, \quad B_{p}^{+}:=B_{j}^{-}, \\
& C_{p}^{-}:=C_{j}^{+}, \quad D_{p}^{-}:=D_{j}^{+}, \quad C_{p}^{+}:=C_{j}^{-}, \quad D_{p}^{+}:=D_{j}^{-} .
\end{aligned}
$$

The equivalence between (iii) and (iv) readily follows.

Remark 5.4. Suppose that (i) and (ii) of Lemma 5.3 hold. Then, we also have

$$
\widetilde{\Delta}_{p}=\frac{\widetilde{D}_{j}}{a_{j} c_{j} f_{j}} \text { and } \widetilde{\Gamma}_{p}=\frac{\widetilde{\Gamma}_{j-1}}{a_{j-1} c_{j-1} f_{j-1} a_{j} c_{j} f_{j}} \quad \text { for any } p, j \text { such that } t_{p}=\vartheta\left(t_{j}\right) .
$$

Remark 5.5. Let us successively consider each of the two cases (57) and (58).

- Suppose that we are in the situation (57). Then, applying formula (59) with $t_{q}=\vartheta\left(t_{q}\right)$ yields $a_{q}=c_{q}=f_{q}=1, d_{q}=\frac{1}{2} b_{q} e_{q}$. The spline space $\mathbb{S}$ is good for design if and only if

$$
\widetilde{B}_{k}, \widetilde{D}_{k}>0 \text { for all } k \leqslant q, \quad \widetilde{\Gamma}_{k}>0 \text { for all } k \leqslant q-1 .
$$

- Suppose that we are in the situation (58). Since $t_{q}=\vartheta\left(t_{q+1}\right)$, in particular the space $\mathbb{E}_{q}$ itself is invariant under $\vartheta$, that is $\mathbb{E}_{q}=\overleftarrow{\mathbb{E}}_{q}$. The spline space $\mathbb{S}$ is now be good for design if and only all conditions (63) are satisfied, plus $\widetilde{\Gamma}_{q}>0$. Given that, with $C_{q}:=C_{q}^{+}=C_{q+1}^{-}$,

$$
\widetilde{\Gamma}_{q}=\frac{1}{a_{q} c_{q} f_{q}}\left(\widetilde{D}_{q}^{2}-C_{q}^{2} \widetilde{B}_{q}^{2}\right)
$$

the additional condition $\widetilde{\Gamma}_{q}>0$ reduces to $\widetilde{D}_{q}-C_{q} \widetilde{B}_{q}>0$.

From the previous subsection and Lemma 5.3 we can derive: 
Lemma 5.6. Assume the spline space $\mathbb{S}$ to be invariant under some reflection $\vartheta$ and to be good for design. Then the B-spline basis is invariant under $\vartheta$, in the sense that, for all $j \in \mathbb{Z}$,

$$
N_{j}=N_{2 q-j-5} \circ \vartheta \text { in case (57), } \quad N_{j}=N_{2 q-j-4} \circ \vartheta \text { in case (58). }
$$

To conclude this subsection, assume that all connection matrices are the same, that is,

$$
M_{k}=\left[\begin{array}{lll}
\alpha & 0 & 0 \\
\beta & \gamma & 0 \\
\delta & \varepsilon & \varphi
\end{array}\right] \quad \text { for all } k \in \mathbb{Z} .
$$

Then, the following result readily follows from Lemma 5.3.

Lemma 5.7. Assume that the two conditions (i) and (ii) of Lemma 5.3 hold, along with (65). Then, the spline space $\mathbb{S}$ is invariant under the reflection $\vartheta$ if and only if

$$
\alpha=\gamma=\varphi=1, \quad \delta=\frac{\beta \varepsilon}{2} .
$$

Remark 5.8. Under the assumption (65), that the spline space $\mathbb{S}$ is invariant under the reflection $\vartheta$ does not guarantee that it is good for design.

\subsection{Splines with prescribed section-spaces}

In this subsection, we assume that the sequence of knots $\mathbb{T}$ is given, along with the bi-infinite sequence of section-spaces $\mathbb{E}_{k}, k \in \mathbb{Z}$, where $\mathbb{E}_{k}$ is an EC-space good for design on $\left[t_{k}, t_{k+1}\right]$. The piecewise Chebyshevian spline space $\mathbb{S}$ depends only on the bi-infinite sequence $M_{k}, k \in \mathbb{Z}$, of connection matrices. A natural question arises: is it always possible to find such a bi-infinite sequence so as to ensuring that $\mathbb{S}$ will be good for design, and how to easily obtain all possibilities?

The answer is positive and we can even build infinitely many such spline spaces good for design. For the rest of this section we assume that the positive diagonal entries $a_{k}, c_{k}, f_{k}, k \in \mathbb{Z}$, have been selected once and for all. In this situation, the only parameters which the spline space $\mathbb{S}$ depends on, are the three bi-infinite sequences of non-diagonal entries $b_{k}, d_{k}, e_{k}, k \in \mathbb{Z}$, and we have to make sure that we select them so as to ensure that $\mathbb{S}$ will be good for design. For instance, we can proceed as explained below. Start by selecting any three bi-infinite sequences of positive numbers

$$
\widetilde{D}_{k}, \widetilde{\Delta}_{k}, \widetilde{\Gamma}_{k}, \quad k \in \mathbb{Z},
$$

with

$$
0<\widetilde{\Gamma}_{k}<\widetilde{D}_{k} \widetilde{\Delta}_{k+1}, \quad k \in \mathbb{Z} .
$$

Given any positive $\widetilde{B}_{k_{0}}$, where $k_{0} \in \mathbb{Z}$ is any integer, step by step, compute the positive numbers $\widetilde{B}_{k}, k \in \mathbb{Z}$, as follows:

$$
\widetilde{B}_{k+1}:=\frac{\widetilde{D}_{k} \widetilde{\Delta}_{k+1}-\widetilde{\Gamma}_{k}}{C_{k}^{+} C_{k+1}^{-} f_{k+1} \widetilde{B}_{k}}, \quad k \geqslant k_{0} ; \quad \widetilde{B}_{k-1}:=\frac{\widetilde{D}_{k-1} \widetilde{\Delta}_{k}-\widetilde{\Gamma}_{k-1}}{C_{k-1}^{+} C_{k}^{-} f_{k} \widetilde{B}_{k}}, \quad k \leqslant k_{0} .
$$

Then, introduce the bi-infinite sequence

$$
\widetilde{E}_{k}:=\frac{\widetilde{\Delta}_{k}+c_{k} \widetilde{D}_{k}}{\widetilde{B}_{k}}, \quad k \in \mathbb{Z} .
$$


Last, define the non-diagonal entries of the connection matrices as follows:

$$
\begin{aligned}
b_{k} & :=\widetilde{B}_{k}-a_{k} A_{k}^{+}-c_{k} A_{k}^{-}, \quad e_{k}:=\widetilde{E}_{k}+c_{k} D_{k}^{+}+f_{k} D_{k}^{-}, \\
d_{k} & :=\widetilde{D}_{k}-e_{k} A_{k}^{-}+D_{k}^{+} \widetilde{B}_{k}+a_{k} B_{k}^{+}-f_{k} B_{k}^{-}, \quad k \in \mathbb{Z} .
\end{aligned}
$$

Theorem 4.1 guarantees that the corresponding spline space $\mathbb{S}$ will automatically be good for design.

As a matter of fact, the expression (53) of the B-spline-like basis in $D \mathbb{S}$ makes it obvious that the last step $(70)$ is not necessary to obtain all splines in $\mathbb{S}$. It can therefore be interesting to view the positive numbers $\widetilde{D}_{k}, \widetilde{\Delta}_{k}, \widetilde{\Gamma}_{k}, k \in \mathbb{Z}$, as alternative parameters defining $\mathbb{S}$. They can even be used as shape parameters, rather than $b_{k}, d_{k}, e_{k}, k \in \mathbb{Z}$. This will be illustrated in some the next sections, depending on the situations and motivations.

If the spline $\mathbb{S}$ is assumed to preserve symmetry, then, similarly, from the discussion in the previous subsection, we can take $\widetilde{D}_{k}, \widetilde{\Delta}_{k}, k \leqslant q ; \widetilde{\Gamma}_{k}, k \leqslant q-1$ or $k \leqslant q$, as possible positive shape parameters.

\section{Symmetric cardinal (geometrically continuous) piecewise Chebyshevian B-splines}

Throughout this section we consider regularly spaced simple knots $t_{k}=k h, k \in \mathbb{Z}$, with $h>0$. The geometrically continuous splines investigated here are the closest to the ordinary cardinal quartic splines in so far as the B-splines are obtained by translation of a symmetric B-spline $N_{0}$, with support $\left[t_{0}, t_{5}\right]$.

\subsection{The results}

In order to be able to modify the knot spacing, we have to start with the null space $\mathbb{E}=\operatorname{ker} L$ of a linear differential operator $L$ of order five

$$
L:=D^{5}+\gamma_{2} D^{3}+\gamma_{0} D
$$

where $\gamma_{0}, \gamma_{2}$ are any real numbers. The space $\mathbb{E}$ contains the constants. In Table 1 , we recall the nine different families in the class $\mathcal{C}$ of all such spaces $\mathbb{E}$, depending on the roots of the characteristic polynomial

$$
p(x):=x^{4}+\gamma_{2} x^{2}+\gamma_{0}
$$

associated with $D \mathbb{E}=\operatorname{ker}\left(D^{4}+\gamma_{2} D^{2}+\gamma_{0} \mathrm{Id}\right)$, as investigated in view of design in [9].

There exists a number $\left.\left.\ell_{L} \in\right] 0,+\infty\right]$ such that $\mathbb{E}$ is an EC-space good for design on $[0, h]$ (i.e., $D \mathbb{E}$ is an EC-space on $[0, h])$ if and only if $h<\ell_{L}$. We refer to it as the critical length for design of the space $\mathbb{E}$. Let us recall that this critical length for design can be expressed with the help of the unique function $\tau$ satisfying

$$
\tau^{(4)}+\gamma_{2} \tau^{\prime \prime}+\gamma_{0} \tau=0, \quad \tau(0)=\tau^{\prime}(0)=\tau^{\prime \prime}(0)=0, \quad \tau^{\prime \prime \prime}(0)=1 .
$$

We then have $[13,9]$

$$
\ell_{L}=\min \left(Z_{\tau}, Z_{\eta}\right), \quad \text { where } \eta:=W\left(\tau, \tau^{\prime}\right) .
$$

In (74), $W\left(\tau, \tau^{\prime}\right)$ stands for the Wronskian of the two functions $\tau, \tau^{\prime}$, and $Z_{\tau}, Z_{\eta}$ for the first zeroes of $\tau$ and $\eta$ on $] 0,+\infty$ [, if any. We can then split the class $\mathcal{C}$ into two subclasses $\mathcal{C}_{1}$ and $\mathcal{C}_{2}$, corresponding to $\ell_{L}=+\infty$ and $\ell_{L}<+\infty$. As is well known, the subclass $\mathcal{C}_{1}$ (i.e., $\mathbb{E}$ is an EC-space good for design on the whole of $\mathbb{R}$ ) is characterised by the fact that the characteristic polynomial (72) has only real roots (cases 1, 2, 6, 7, which we will refer to as the real cases). 


\begin{tabular}{|c|l|l|}
\hline Case & \multicolumn{1}{|c|}{$p(x)$} & \multicolumn{1}{|c|}{ a basis of $\mathbb{E}$} \\
\hline 1 & $\left(x^{2}-a^{2}\right)\left(x^{2}-b^{2}\right), a, b>0, a \neq b$ & $1, \cosh (a x), \sinh (a x), \cosh (b x), \sinh (b x)$ \\
\hline 2 & $\left(x^{2}-a^{2}\right) x^{2}, a>0$ & $1, x, x^{2}, \cosh (a x), \sinh (a x)$ \\
\hline 3 & $\left(x^{2}-a^{2}\right)\left(x^{2}+b^{2}\right), a, b>0$ & $1, \cosh (a x), \sinh (a x), \cos (b x), \sin (b x)$ \\
\hline 4 & $x^{2}\left(x^{2}+b^{2}\right), b>0$ & $1, x, x^{2}, \cos (b x), \sin (b x)$ \\
\hline 5 & $\left(x^{2}+a^{2}\right)\left(x^{2}+b^{2}\right), a, b>0, a \neq b$ & $1, \cos (a x), \sin (a x), \cos (b x), \sin (b x)$ \\
\hline 6 & $\left(x^{2}-a^{2}\right)^{2}, a>0$ & $1, \cosh (a x), \sinh (a x), x \cosh (a x), x \sinh (a x)$ \\
\hline 7 & $x^{4}$ & $1, x, x^{2}, x^{3}, x^{4}$ \\
\hline 8 & $\left(x^{2}+b^{2}\right)^{2}, b>0$ & $1, \cos (b x), \sin (b x), x \cos (b x), x \sin (b x)$ \\
\hline 9 & $x^{4}+2\left(b^{2}-a^{2}\right) x^{2}+\left(a^{2}+b^{2}\right)^{2}, a, b>0$ & $\begin{array}{l}1, \cosh (a x) \cos (b x), \cosh (a x) \sin (b x), \\
\sinh (a x) \cos (b x), \sinh (a x) \sin (b x)\end{array}$ \\
\hline
\end{tabular}

Table 1: The class $\mathcal{C}$ of all spaces $\mathbb{E}=\operatorname{ker} L$

Subsequently we assume that $h<\ell_{L}$. For each $k$, the section-space $\mathbb{E}_{k}$ is the restriction of $\mathbb{E}$ to $\left[t_{k}, t_{k+1}\right]=[k h,(k+1) h]$, and it is therefore an EC-space good for design on $\left[t_{k}, t_{k+1}\right]$. For each $k \in \mathbb{Z}$, the Bernstein-like basis $\left(V_{0}^{k}, V_{1}^{k}, V_{2}^{k}, V_{3}^{k}\right)$ in $D \mathbb{E}_{k}$ is obtained by translation of the restrictions to $\left[t_{0}, t_{1}\right]=[0, h]$ of the Bernstein-like basis $\left(V_{0}, V_{1}, V_{2}, V_{3}\right)$ relative to $(0, h)$ in $D \mathbb{E}$ defined by the conditions

$$
\begin{aligned}
& V_{3}(0)=V_{3}{ }^{\prime}(0)=V_{3}{ }^{\prime \prime}(0)=0, \quad V_{3}(h)=1, \\
& V_{2}(0)=V_{2}{ }^{\prime}(0)=0, \quad V_{2}(h)=0, V_{2}{ }^{\prime}(h)=-1, \\
& V_{0}:=V_{3}(h-.), \quad V_{1}:=V_{2}(h-.) .
\end{aligned}
$$

We can therefore drop all indices in (28), for

$$
\begin{aligned}
& A_{k}^{-}=A_{k}^{+}=A:=V_{3}^{\prime}(h), \quad B_{k}^{-}=B_{k}^{+}=B:=V_{3}^{\prime \prime}(h), \\
& C_{k}^{-}=C_{k}^{+}=C:=V_{2}^{\prime \prime}(0), \quad D_{k}^{-}=D_{k}^{+}=D:=V_{2}^{\prime \prime}(h), \quad k \in \mathbb{Z} .
\end{aligned}
$$

Let us recall that the quantities $A, B, C, D$ depend on at most three parameters: the length $h$, and at most two parameters involved in the space $\mathbb{E}$ (see Table 1 ).

Conditions (i) and (ii) of Lemma 5.3 being satisfied with any integer $q$ and both (57) and (58), in order to be in the cardinal symmetric situation it is necessary and sufficient to choose connection matrices of the form (see Lemma 5.7)

$$
M_{k}:=M:=\left[\begin{array}{lll}
1 & 0 & 0 \\
\beta & 1 & 0 \\
\frac{\beta \varepsilon}{2} & \varepsilon & 1
\end{array}\right] \quad \text { for all } k \in \mathbb{Z}
$$

Denote by $\mathbb{S}(L ; M ; h)$ the corresponding space of geometrically continuous $L$-splines. It depends on the parameters $h, \beta, \varepsilon$, and of course of $L$. Splines in $\mathbb{S}(L ; M ; h)$ are $F^{3}$ in the usual sense of continuity of the Frenet frames of order three and of the first two curvatures. Due to the cardinal situation, the quantities $\widetilde{B}_{k}, \widetilde{E}_{k}, \widetilde{D}_{k}, \widetilde{\Gamma}_{k}, \widetilde{\Delta}_{k}$ introduced in (30) and (51) are independent of $k$. Subsequently, we can therefore drop the indices. We thus have

$$
\widetilde{B}=\beta+2 A, \quad \widetilde{E}=\varepsilon-2 D,
$$

Due to the symmetry of the space $\mathbb{E}$, the entry $\beta \varepsilon / 2$ implies that

$$
\widetilde{D}=\widetilde{\Delta}=\frac{\widetilde{B} \widetilde{E}}{2} .
$$


Accordingly,

$$
\widetilde{\Gamma}=\widetilde{D} \widetilde{\Delta}-C^{2} \widetilde{B}=\frac{\widetilde{B}^{2}}{4}(\widetilde{E}-2 C)(\widetilde{E}+2 C) .
$$

On account of (77) and of the positivity of $C$, conditions (29) reduce to

$$
\widetilde{B}>0, \quad \widetilde{E}>2 C .
$$

Due to (76), we can thus state:

Theorem 6.1. Given $h<\ell$, the spline space $\mathbb{S}(L ; M ; h)$ is good for design if and only if the two conditions below are satisfied

$$
\beta>-2 A, \quad \varepsilon>2(C+D) .
$$

Assume that conditions (80) are satisfied. Then, from (53), we can see that, as a (symmetric) B-spline-like $Q_{0} \in D \mathbb{S}(L ; M ; h)$, with support $[0,4 h]$, we can take

$$
Q_{0}(x)=\left\{\begin{array}{l}
V_{3}(x) \quad \text { for } x \in[0, h], \\
V_{0}(x-h)+\widehat{B} V_{1}(x-h)+\frac{1}{2} \widehat{B} \widehat{E} V_{2}(x-h)+\widehat{E} V_{3}(x-h) \quad \text { for } x \in[h, 2 h], \\
\widehat{E} V_{0}(x-2 h)+\frac{1}{2} \widehat{B} \widehat{E} V_{1}(x-2 h)+\widehat{B} V_{2}(x-2 h)+V_{3}(x-2 h) \quad \text { for } x \in[2 h, 3 h], \\
V_{0}(x-3 h) \quad \text { for } x \in[3 h, 4 h],
\end{array}\right.
$$

where

$$
\widehat{B}:=\widetilde{B}>0, \quad \widehat{E}:=\frac{\widetilde{E}}{C}>2 .
$$

It should be observed that the two numbers $\widehat{B}, \widehat{E}$, depend not only on the matrix $M$ (that is, on the parameters $\beta, \varepsilon$ ), but also of $L$ (that is, on the parameters defining $\mathbb{E}$, see Table 1 ), and on $h$. The B-spline $N_{0} \in \mathbb{S}(L ; M ; h)$ with support $[0,5 h]$ is then obtained as

$$
N_{0}(x)=\frac{\int_{0}^{x} Q_{0}(t) d t-\int_{h}^{x} Q_{0}(t-h) d t}{\int_{0}^{4 h} Q_{0}(t) d t}, \quad x \in \mathbb{R} .
$$

With a view to analyzing the shape effects it is convenient to revisit Proposition 52 as follows.

Theorem 6.2. The operator $L$ in (71) being given, let $\ell_{L}$ be the critical length for design of the space $\mathbb{E}=\operatorname{ker} L$. Take any positive number $h<\ell_{L}$. Then, for any two parameters $\widehat{B}>0$ and $\widehat{E}>2$, the set composed of all functions $S$ of the form

$$
S(x):=\sum_{j \in \mathbb{Z}} a_{j} N_{0}(x-j h), \quad x \in \mathbb{R},
$$

where the $a_{j}$ 's are any real numbers, and where $N_{0}$ is defined by (82) and (81), is the spline space $\mathbb{S}(L ; M ; h)$, the matrix $M$ in (65) being obtained with

$$
\beta:=\widehat{B}-2 A, \quad \varepsilon:=C \widehat{E}+2 D .
$$

Moreover the spline space $\mathbb{S}(L ; M ; h)$ is good for design. Conversely, any spline space $\mathbb{S}(L ; M ; h)$ which is good for design is of the form described above.

In other words, the two parameters $\widehat{B}, \widehat{E}$ can be taken as shape parameters instead of $\beta, \varepsilon$. For fixed $\widehat{B}, \widehat{E}$, the corresponding entries $\beta, \varepsilon$ of $M$ depend on both $L$ and $h$. 


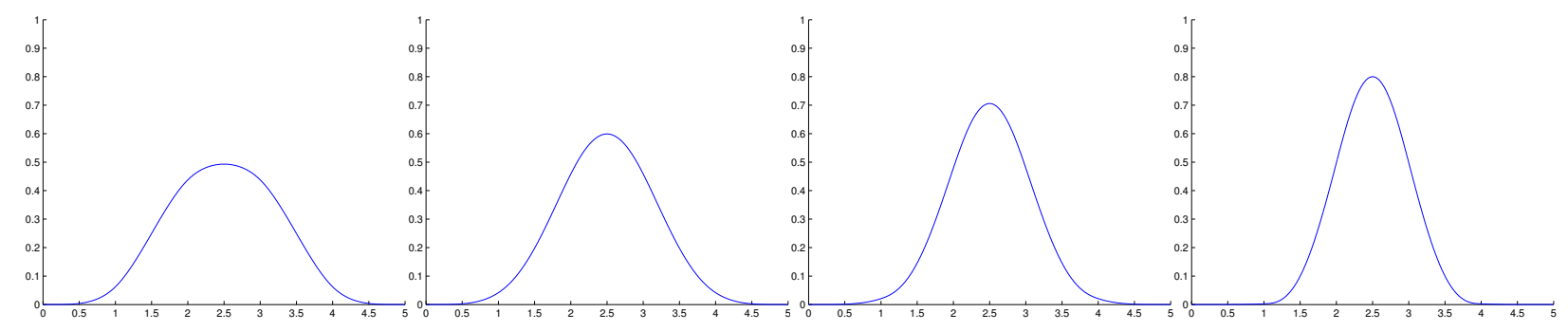

Figure 1: The symmetric $C^{2}, F^{3}$, quartic B-spline $N_{0}$ given by (82), (81), and (84), with $\widehat{B}=6$ (i.e., $\beta=0$ ). From left to right $\widehat{E}=2.01 ; 4$ (usual quartic B-spline); $10 ; 100$.

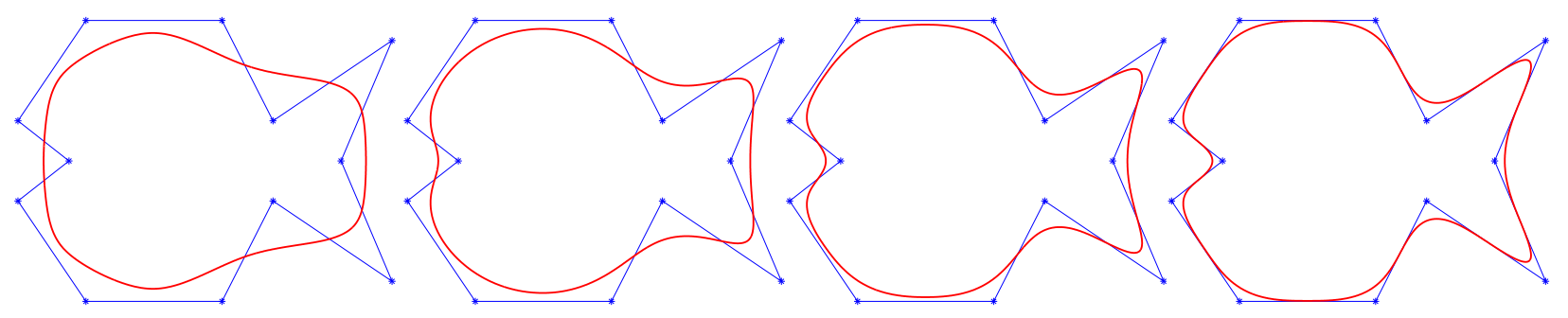

Figure 2: $C^{2}, F^{3}$ quartic spline curves with $\widehat{B}=6$ and, from left to right $\widehat{E}=2.01 ; 4 ; 10 ; 100$.

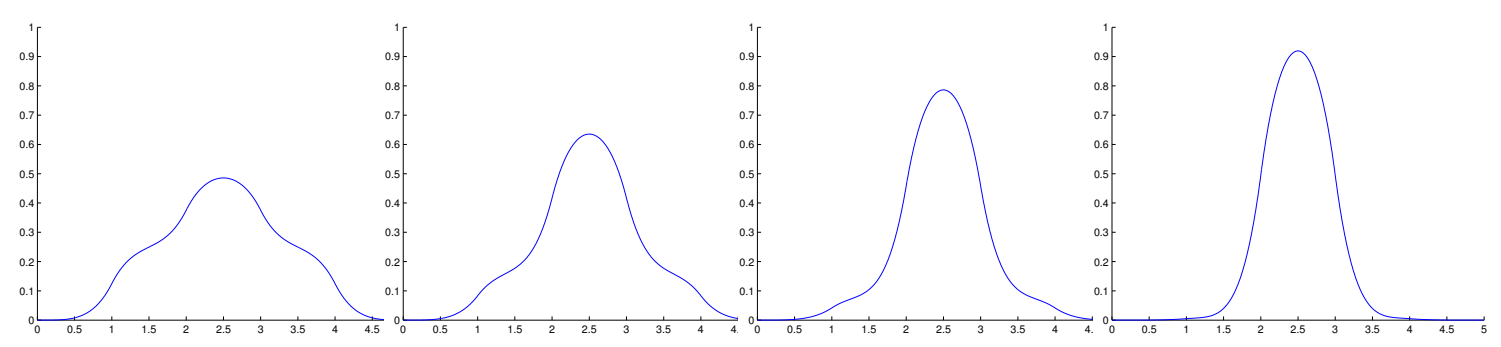

Figure 3: The symmetric $C^{1}, F^{3}$, quartic B-spline $N_{0}$, with $\widehat{B}=0.01$. From left to right $\widehat{E}=2.01 ; 4 ; 10$; 100.

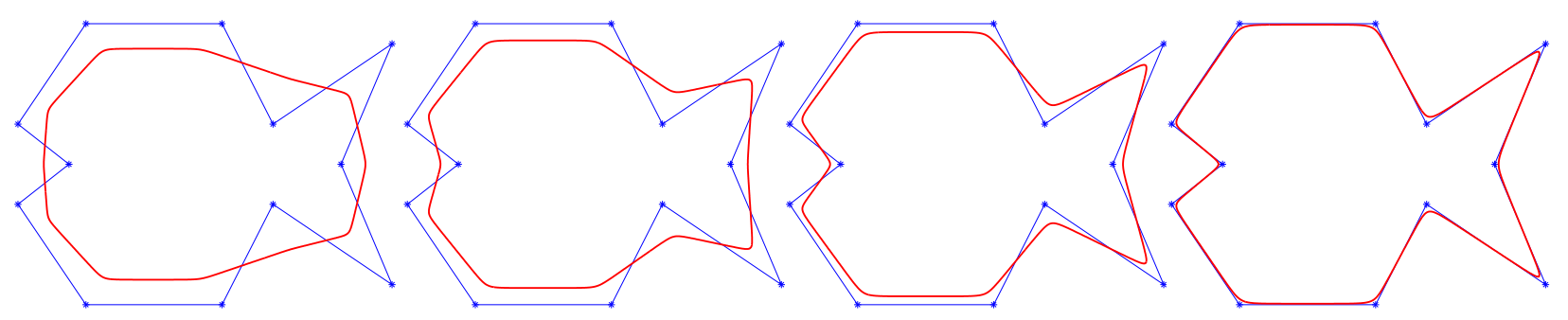

Figure 4: $C^{1}, F^{3}$ quartic spline curves with $\widehat{B}=0.01$ and, from left to right, $\widehat{E}=2.01 ; 4 ; 10 ; 100$. 


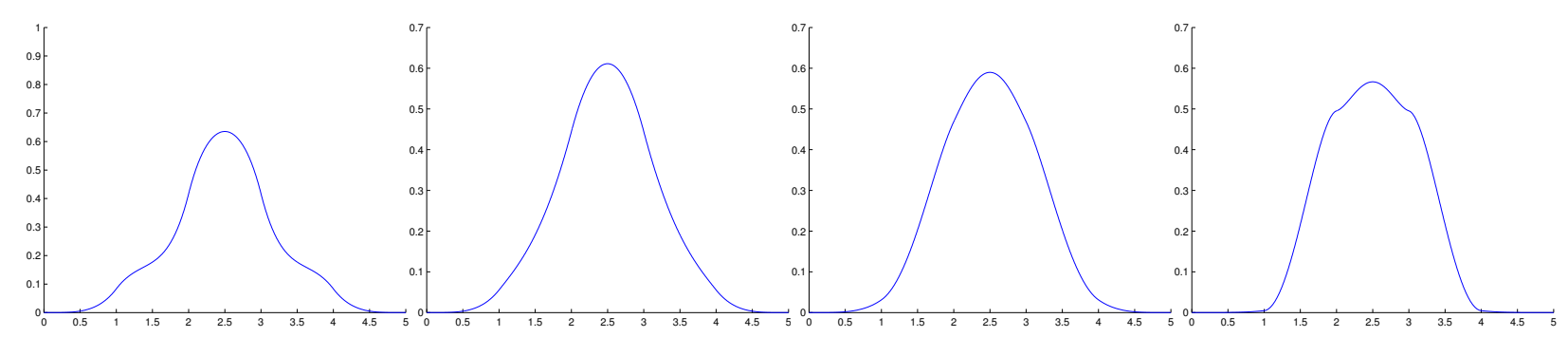

Figure 5: The symmetric $C^{1}, F^{3}$, quartic B-spline $N_{0}$, with $\widehat{E}=4$, and from left to right $\widehat{B}=0,01$; $6 ; 10 ; 100$.

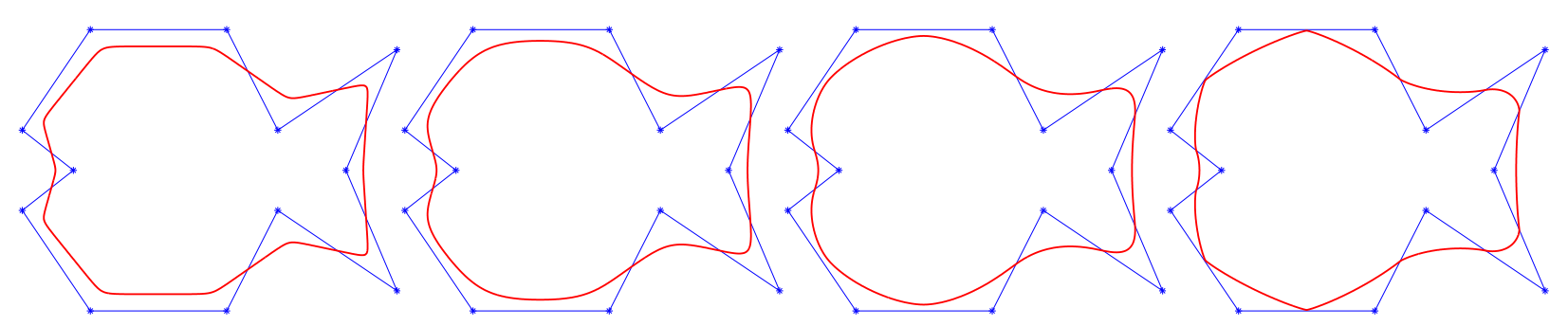

Figure $6: C^{1}, F^{3}$ quartic spline curves with $\widehat{E}=4$ and, from left to right, $\widehat{B}=0,01 ; 6 ; 10 ; 100$.

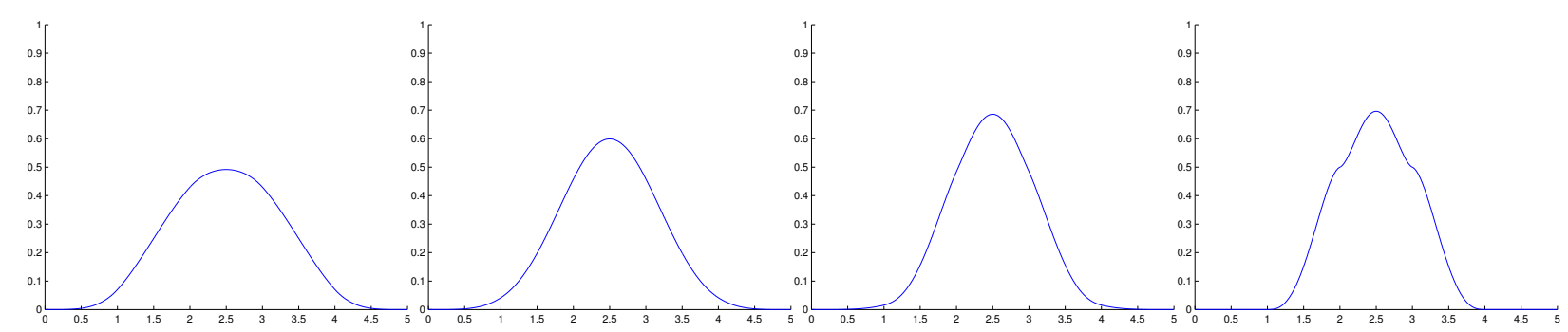

Figure 7: The symmetric $C^{1}, G^{3}$, quartic B-spline $N_{0}$ given by (??), (??), (84), and (86), obtained with, from left to right, $\widehat{B}=4.67 ; 6$ (usual quartic B-spline); $10 ; 100$.

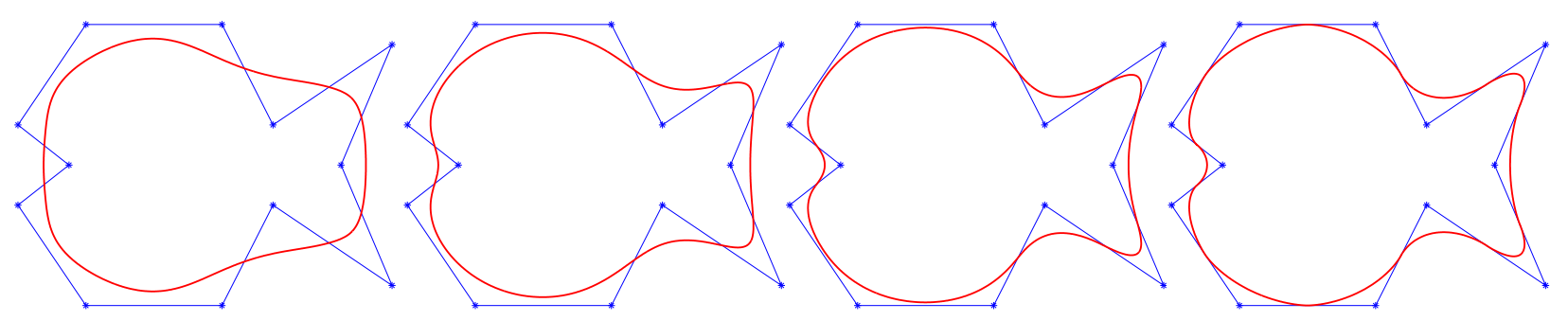

Figure 8: $C^{1}, G^{3}$, quartic spline curves with, from left to right, $\widehat{B}=4.67 ; 6 ; 10 ; 100$. 


\subsection{Cardinal symmetric geometrically continuous polynomial B-splines}

In this subsection we apply Theorem 6.2 in the case where $\mathbb{E}=\mathcal{P}_{4}$ is the space of all quartic polynomials on $\mathbb{R}$, thus corresponding to $\gamma_{0}=\gamma_{2}=0$ in (71). We put special emphasis on illustrating this case which is the closest to the classical quartic splines, and which already permits many interesting shape effects.

Since no parameter is provided by the space $\mathcal{P}_{4}$, the spline space $\mathbb{S}\left(D^{5}, M, h\right)$ depends on the only two free parameters $\beta, \varepsilon$ defining the matrix $M$ in (65) and on $h$. Being interested in spline design, without loss of generality we can assume that $h=1$. Indeed, this does not reduce the class of parametric spline curves we can create. The B-spline $N_{0}$ is given by (82) and (81) with

$$
V_{3}(x)=x^{3}, \quad V_{2}(x)=x^{2}-x^{3}, \quad x \in \mathbb{R},
$$

and, therefore $A=3, C=2, D=-4$. Rather than $\beta, \varepsilon$, we can say that $\mathbb{S}$ depends on the two free parameters $\widehat{B}>0, \widehat{E}>2$, with

$$
\widehat{B}=\beta+6>0, \quad \widehat{E}=\frac{\varepsilon+8}{2}>2 .
$$

Special requirements on the regularity of the curves can easily be translated into requirements on $\widehat{B}>0, \widehat{E}>2$ via (85). For instance $C^{2}$ splines are obtained for $\widehat{B}=6$, while the ordinary $C^{3}$ quartic splines are obtained for $\widehat{B}=6$ and $\widehat{E}=4$.

The illustrations provided (Figs. 1-8) show only some of the possible shape effects produced by these two parameters. In Figs. 1 and 2, we take $\widehat{B}=6$, and we show the effect of increasing $\widehat{E}$ from $2^{+}$to $+\infty$, respectively on the $C^{2}$ B-spline $N_{0}$ and one a parametric $C^{2}$ spline curve with prescribed control points. For comparison, similar illustrations are presented in Figs. 3 and 4, but now with $\widehat{B}=0.01$. In both cases we observe that increasing the values of $\widehat{E}$ pushes the curve towards the control polygon, however in a different way. For $\widehat{B}=0.01$, for $\widehat{E} \rightarrow+\infty$ we somehow obtain " $C^{1}$ piecewise affine curves"!

In the present context, $G^{3}$ splines correspond to $\varepsilon=3 \beta$. Equivalently, due to (85)

$$
\text { splines in } \mathbb{S} \text { are } G^{3} \Leftrightarrow \widehat{E}=\frac{3}{2} \widehat{B}-5 \text {. }
$$

When (86) holds, the spline space depends on the only parameter $\widehat{B}$ which is now required to satisfy $\widehat{B}>\frac{14}{3}$ in order to ensure (80). Corresponding illustrations can be found in Figs. 7 and 8 .

\subsection{Other cardinal symmetric geometrically continuous B-splines}

In this subsection, $\mathbb{E}=\operatorname{ker} L$ is any of the spaces indicated in Table 1 other than the quartic space $\mathcal{P}_{4}$. We could of course provide illustrations similar to Figs. $1-8$ in each case, the only difference being that the effects of the two parameters $\widehat{B}, \widehat{E}$ investigated in the previous subsection would be combined with those permitted by the parameter(s) on which the space $\mathbb{E}$ depends [9]. Instead, we prefer to more precisely point out the differences between the two subclasses $\mathcal{C}_{1}$ and $\mathcal{C}_{2}$ of the class $\mathcal{C}$, which respectively correspond to the real cases (Cases $1 ; 2 ; 6 ; 7$ ) for which all roots of the characteristic polynomial (72) are real) and to the non-real ones (Cases $3 ; 4 ; 5 ; 8 ; 9$ ).

With this in mind, we need to mention that the special case of parametrically continuous splines (i.e., the matrix $M$ in (75) is the identity matrix $I_{3}$ ) was already addressed in [10], where the following results was obtained.

Theorem 6.3. The differential operator $L$ in (71) and a positive $h$ being given, with $h<\ell_{L}$, the following properties are equivalent: 
(i) the space $\mathbb{S}\left(L ; I_{3} ; h\right)$ of all $C^{3} L$-splines is a piecewise Chebyshevian spline space good for design;

(ii) $A>0$, and $C+D<0$;

(iii) the knot spacing satisfies

$$
\tau^{\prime}(h)>0, \quad \psi(h)<0, \quad \text { where } \psi(h):=\eta^{\prime}(h)+\tau(h) .
$$

(iv) the knot spacing satisfies $h<\frac{\pi}{\mu_{L}}$, where $\mu_{L}$ denotes the maximum of the imaginary parts of all roots of the characteristic polynomial $p$ in (72).

Observe that the equivalence between (i) and (ii) is simply Theorem 6.1 with $\beta=\varepsilon=0$. The reader is referred to [10] for all details, in particular concerning the quantities $A, B, C, \ldots$ which can be expressed in terms of the functions $\tau$ and $\eta$ introduced in (73) and (74). For instance, we have

$$
A=\frac{\tau^{\prime}(h)}{\tau(h)}, \quad C+D=-\frac{\psi(h)}{\eta(h)},
$$

which explains why (ii) can be replaced by (iii).

Remark 6.4. It is important to comment on Theorem 6.3 with a view to compare the real and the non-real cases. Given a space $\mathbb{E}=\operatorname{ker} L$ in the class $\mathcal{C}$, and given any positive $h$, from (74), we know that the assumption $h<\ell_{L}$ can be replaced by

$$
h<\min \left(Z_{\tau}, Z_{\eta}\right) .
$$

The three requirements $h<\ell_{L}, A>0, C+D<0$ together can thus be condensed into the single one below:

$$
h<\min \left(Z_{\tau^{\prime}}, Z_{\psi}\right) .
$$

From Theorem 6.1 (or Theorem 6.2) we know that condition (88) is the necessary and sufficient condition for the existence of infinitely many connection matrices $M$ of the form (75) for which the space $\mathbb{S}(L ; M ; h)$ of all associated cardinal geometrically continuous $L$-splines will be good for design. The stronger requirement (89) is the necessary and sufficient condition for the identity matrix $I_{3}$ to be one among these infinitely many possible matrices.

Let us first assume that we are in any of the real cases. From (iv) of Theorem 6.3 we can deduce that the spline space $\mathbb{S}\left(L ; I_{3} ; h\right)$ is good for design whatever the positive number $h$. As a matter of fact, in that case, this is a well-known fact since the space $\mathbb{E}$ is an EC-space good for design on the whole of $\mathbb{R}$ : indeed, whatever $h>0$, the space $\mathbb{S}\left(L ; I_{3} ; h\right)$ is a Chebyshevian spline space. From (ii) we can also express this as follows: $\min \left(Z_{\tau^{\prime}}, Z_{\psi}\right)=+\infty$, that is, whatever $h>0$, we have $A>0$ and $C+D<0$.

By contrast, in any non-real case, condition (89) is always strictly stronger than (89), which means that we can obtain cardinal geometrically continuous refinable B-spline bases without the two conditions $A>0$ and $C+D<0$ being satisfied. This will be illustrated in the example below.

Example 6.5. Here we assume that $\mathbb{E}=\operatorname{ker} L$ belongs to Case 9 , and therefore $L$ depends on two positive parameters $a, b$. Then, $Z_{\eta}=Z_{\psi}=+\infty$, and $Z_{\tau^{\prime}}=\frac{\pi}{b}[9,10]$. On the other hand, we know that $\ell_{L}=Z_{\tau}<\frac{3 \pi}{2 b}$. From now on, we require that $h=1$. Let us first determine the set $\Omega$ composed of all pairs $(a, b)$ of positive numbers such that $\ell_{L}>1$. From the expression of $\tau$ recalled in Table 2 (see next section), it is easily seen that, for $(a, b)$ to belong to $\Omega$, it is necessary that $b<x_{0}$, where $x_{0}$ is characterised

$$
\pi<x_{0}<\frac{3 \pi}{2}, \quad \tan x_{0}=x_{0} .
$$

Besides, when $b<x_{0}$, two different situations have to be considered. 


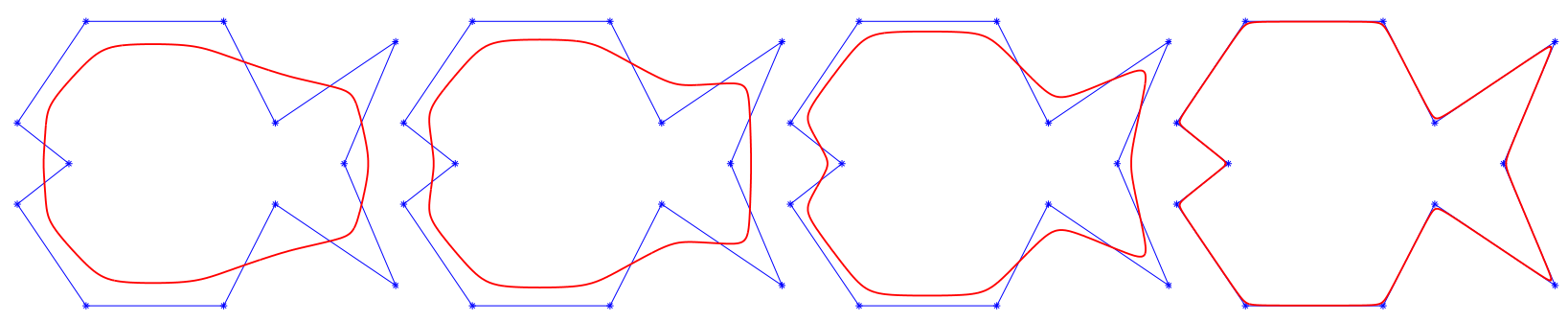

Figure 9: $C^{3}$ cardinal splines with all sections taken from the same space of Case 9, and with $h=1$, and $b=3$. From left to right: $a=0.1 ; 1 ; 2 ; 6$.

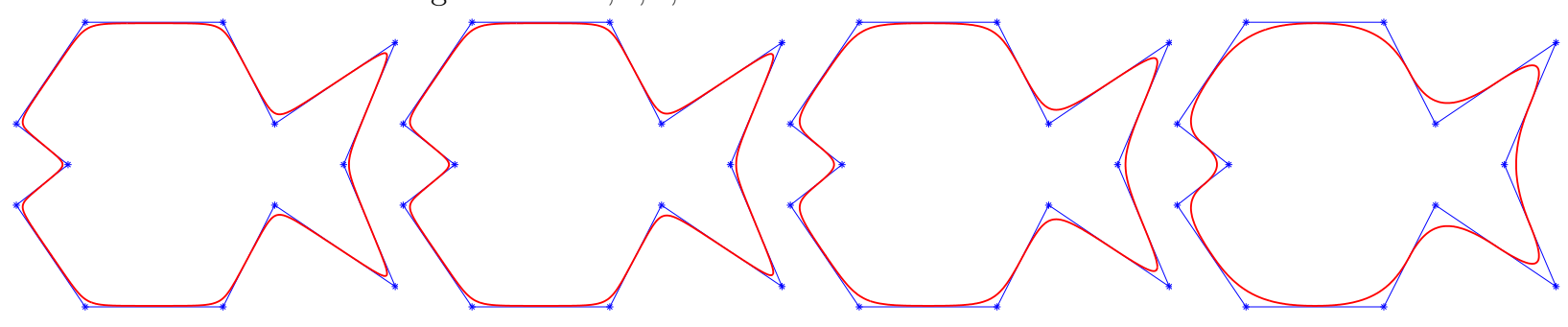

Figure 10: Geometrically continuous cardinal splines with all sections taken from the same space of Case 9 , and with $\widehat{B}=0.01, \widehat{E}=100$. From left to right: $b=3, a=2 ; b=3.2, a=2 ; b=4, a=3.4$, and $b=4.49, a=0.21$

1) First assume that $b \leqslant \pi$ : then, for each $a>0$ we have $(a, b) \in \Omega$.

2) We now assume that $\pi<b<x_{0}$. Then,

$$
(a, b) \in \Omega \quad \Leftrightarrow \quad \frac{1}{a} \tanh a>\frac{1}{b} \tan b .
$$

For each pair $(a, b) \in \Omega$, there exist infinitely many different matrices $M$ in (75) such that the spline space $\mathbb{S}(L ; M ; 1)$ is good for design. However only when $b<\pi$, the identity matrix $I_{3}$ is one of these matrices. We can even state that only for $b<\pi$ we can obtain $C^{2}$ splines good for design. Recall that when $a \rightarrow+\infty$, the $C^{3}$ spline curve converges to the control polygon, all the more efficiently as $b$ is closer to $\pi^{-}$, see [10]. The variations in shape for increasing values of $a$ are shown in Fig. 9 with $b=3$.

Two numbers $\widehat{B}>0, \widehat{E}>2$ being given, the spline curve obtained via Theorem 6.2 evolves in a continuous way as $(a, b)$ ranges over $\Omega$. This continuity can be seen in Fig. 10 . The effects of the two parameters $\widehat{B}>0, \widehat{E}>2$ (addressed for geometrically continuous polynomial splines in the previous subsection) can be combined with those of the two parameters $a, b$. For instance, the values $\widehat{B}=0.01$ and $\widehat{E}=100$ help the spline curve approach the control polygon for smaller values of $a$ than in the $C^{3}$ cases (compare the case $b=3, a=2$ in Figs. 9 and 10). For higher values of $a$, the introduction of $\widehat{B}=0.01, \widehat{E}=100$ does not change the curve. Our final comment concern the case $b>\pi$. As $b$ get closer to $x_{0}$ (located in $] 4.4934,4.4935[$ ), condition (90) requires $a$ to be closer and closer to 0 . For instance, take $b=4$. Then the upper bound for $a$ making condition (90) valid is located in ]3.447,3.448[, while for $b=4.49$, it lies in ]0.214, $0.215[$. This explains the values $a=3,4$ and $a=0.21$ taken in the right two pictures of Fig. 10 to show the limit curves. 


\section{$7 \quad$ Parametrically continuous mixed L-splines}

As already mentioned, in [10] we investigated parametrically continuous splines with simple equispaced knots and with all sections taken from the same given space of the class $\mathcal{C}$. We could point out both the efficiency of some parameters in producing shape variations and also the great variety of possible shapes. A natural question arises: is it possible to increase this variety by designing with splines mixing several spaces of the class $\mathcal{C}$, while preserving the $C^{3}$ nature of the splines? This is the question addressed in the present section.

Though we are no longer within the cardinal case, we can take advantage of the invariance under reflection / translation within the class $\mathcal{C}$. Subsequently, we consider that the space $\mathbb{S}$ of $C^{3}$ splines is defined by pairs $(\mathbb{E}, h)$, where $\mathbb{E}=\operatorname{ker} L$ belongs to the class $\mathcal{C}$, and where $0<h<\ell_{L}$. That the section-space $\mathbb{E}_{k}$ is attached to the pair $(\mathbb{E}, h)$ means both that $t_{k+1}-t_{k}=h$ and that $\mathbb{E}_{k}$ is the restriction of $\mathbb{E}$ to $\left[t_{k}, t_{k+1}\right]$. Then, the corresponding numbers introduced in (28) satisfy $A_{k}^{+}=A_{k+1}^{-}$, $B_{k}^{+}=B_{k+1}^{-}, C_{k}^{+}=C_{k+1}^{-}, D_{k}^{+}=D_{k+1}^{-}$, and they are the same for all section-spaces attached to $(\mathbb{E}, h)$. Subsequently, we will denote them by $A, B, C, D$, for all sections attached to $(\mathbb{E}, h)$. We will similarly use $A^{*}, B^{*}, C^{*}, D^{*}$ for all sections attached to another pair $\left(\mathbb{E}^{*}, h^{*}\right)$, and so forth. The quantity $\widetilde{B}_{k}$ in $(30)$, which depends on the two section-spaces $\mathbb{E}_{k-1}, \mathbb{E}_{k}$ will be denoted as $\widetilde{B}(\mathbb{E}, \mathbb{E})$, $\widetilde{B}\left(\mathbb{E}, \mathbb{E}^{*}\right), \widetilde{B}\left(\mathbb{E}^{*}, \mathbb{E}\right), \widetilde{B}\left(\mathbb{E}^{*}, \mathbb{E}^{*}\right)$, depending on the case, and similar notations will be used for $\widetilde{D}_{k}, \widetilde{\Delta}_{k}$. In the latter notations, we have omitted $h, h^{*}, \ldots$ for the sake of simplicity. According to a similar convention, the quantity $\widetilde{\Gamma}_{k}$, which depends on the three consecutive section-spaces $\mathbb{E}_{k-1}, \mathbb{E}_{k}, \mathbb{E}_{k+1}$, will be denoted as $\widetilde{\Gamma}(\mathbb{E}, \mathbb{E}, \mathbb{E})$, or $\widetilde{\Gamma}\left(\mathbb{E}, \mathbb{E}, \mathbb{E}^{*}\right)$, and so forth.

With the previous notations, the following interesting result can be derived from Theorem 4.1.

Theorem 7.1. Given a family $\mathcal{F}$ of pairs $(\mathbb{E}, h)$, where $\mathbb{E}=\operatorname{ker} L$ is a space in the class $\mathcal{C}$ and where $0<h<\ell_{L}$, the following properties are equivalent:

(i) all spaces $\mathbb{S}$ of $C^{3}$ piecewise Chebyshevian splines obtained by mixing any pairs of the family $\mathcal{F}$ are good for design;

(ii) any pair $(\mathbb{E}, h) \in \mathcal{F}$ satisfies (ii) of Theorem 6.3, and any two pairs $(\mathbb{E}, h),\left(\mathbb{E}^{*}, h^{*}\right)$ in the family $\mathcal{F}$ satisfy

$$
C+D<\frac{B^{*}-B}{A^{*}+A}<-\left(C^{*}+D^{*}\right)
$$

Proof. The necessary and sufficient conditions (29) concern at most three consecutive section-spaces. Using the notations introduced above, condition (i) is thus satisfied if and only if any three pairs $(\mathbb{E}, h),\left(\mathbb{E}^{*}, h^{*}\right),\left(\mathbb{E}^{* *}, h^{* *}\right)$ in the family $\mathcal{F}$ satisfy

$$
\begin{aligned}
& \widetilde{B}(\mathbb{E}, \mathbb{E})>0, \quad \widetilde{D}(\mathbb{E}, \mathbb{E})>0, \quad \widetilde{\Gamma}(\mathbb{E}, \mathbb{E}, \mathbb{E})>0, \\
& \widetilde{B}\left(\mathbb{E}, \mathbb{E}^{*}\right)>0, \quad \widetilde{D}\left(\mathbb{E}, \mathbb{E}^{*}\right)>0, \quad \widetilde{\Gamma}\left(\mathbb{E}, \mathbb{E}, \mathbb{E}^{*}\right)>0, \quad \widetilde{\Gamma}\left(\mathbb{E}, \mathbb{E}^{*}, \mathbb{E}\right)>0, \quad \widetilde{\Gamma}\left(\mathbb{E}, \mathbb{E}^{*}, \mathbb{E}^{*}\right)>0, \\
& \widetilde{\Gamma}\left(\mathbb{E}, \mathbb{E}^{*}, \mathbb{E}^{* *}\right)>0 .
\end{aligned}
$$

The first line in (92) corresponds to the necessary and sufficient condition for the space $\mathbb{S}$ to be good for design when all sections are attached to the same pair $(\mathbb{E}, h)$, and was recalled in Theorem 6.3. Without loss of generality, we can thus directly assume that each pair $(\mathbb{E}, h) \in \mathcal{F}$ satisfies $A>0$, $C+D<0$. The various quantities in the second line of (92) can be expressed as follows:

$$
\begin{aligned}
& \widetilde{B}\left(\mathbb{E}, \mathbb{E}^{*}\right)=A+A^{*}=\widetilde{B}\left(\mathbb{E}^{*}, \mathbb{E}\right), \\
& \widetilde{D}\left(\mathbb{E}, \mathbb{E}^{*}\right)=B-B^{*}-D^{*} \widetilde{B}\left(\mathbb{E}, \mathbb{E}^{*}\right)=\widetilde{\Delta}\left(\mathbb{E}^{*}, \mathbb{E}\right), \\
& \widetilde{\Gamma}\left(\mathbb{E}, \mathbb{E}^{*}, \mathbb{E}^{*}\right)=\widetilde{D}\left(\mathbb{E}, \mathbb{E}^{*}\right) \widetilde{D}\left(\mathbb{E}^{*}, \mathbb{E}^{*}\right)-C^{* 2} \widetilde{B}\left(\mathbb{E}, \mathbb{E}^{*}\right) \widetilde{B}\left(\mathbb{E}^{*}, \mathbb{E}^{*}\right)=\widetilde{\Gamma}\left(\mathbb{E}^{*}, \mathbb{E}^{*}, \mathbb{E}\right), \\
& \widetilde{\Gamma}\left(\mathbb{E}, \mathbb{E}^{*}, \mathbb{E}\right)=\widetilde{D}\left(\mathbb{E}, \mathbb{E}^{*}\right)^{2}-C^{* 2} \widetilde{B}\left(\mathbb{E}, \mathbb{E}^{*}\right)^{2}
\end{aligned}
$$


In particular, all quantities $\widetilde{B}\left(\mathbb{E}, \mathbb{E}^{*}\right)$ are automatically positive. Accordingly, the last line in (93) shows that all quantities $\widetilde{D}\left(\mathbb{E}, \mathbb{E}^{*}\right)$ and $\widetilde{\Gamma}\left(\mathbb{E}, \mathbb{E}^{*}, \mathbb{E}\right)$ are positive if and only if

$$
\widetilde{D}\left(\mathbb{E}, \mathbb{E}^{*}\right)>C^{*} \widetilde{B}\left(\mathbb{E}, \mathbb{E}^{*}\right) \text { for any two pairs }(\mathbb{E}, h),\left(\mathbb{E}^{*}, h^{*}\right) \text { in } \mathcal{F} \text {. }
$$

Suppose that (94) holds true. Given that

$$
\widetilde{\Gamma}\left(\mathbb{E}, \mathbb{E}^{*}, \mathbb{E}^{* *}\right)=\widetilde{D}\left(\mathbb{E}, \mathbb{E}^{*}\right) \widetilde{D}\left(\mathbb{E}^{* *}, \mathbb{E}^{*}\right)-C^{* 2} \widetilde{B}\left(\mathbb{E}, \mathbb{E}^{*}\right) \widetilde{B}\left(\mathbb{E}^{*}, \mathbb{E}^{* *}\right),
$$

a double application of $(94)$ proves the positivity of all quantities $\widetilde{\Gamma}\left(\mathbb{E}, \mathbb{E}^{*}, \mathbb{E}^{*}\right)$, and, therefore, of all $\widetilde{\Gamma}\left(\mathbb{E}, \mathbb{E}, \mathbb{E}^{*}\right)$, and $\widetilde{\Gamma}\left(\mathbb{E}, \mathbb{E}^{*}, \mathbb{E}^{*}\right)$ as special cases.

In other words, the positivity of all other quantities in (92) reduces to (94). Taking account of the first two lines in $(93)$, in $(94)$ the inequality $\widetilde{D}\left(\mathbb{E}, \mathbb{E}^{*}\right)>C^{*} \widetilde{B}\left(\mathbb{E}, \mathbb{E}^{*}\right)$ can be replaced by

$$
\frac{B^{*}-B}{A^{*}+A}<-\left(C^{*}+D^{*}\right)
$$

Exchanging the roles of $\mathbb{E}$ and $\mathbb{E}^{*}$ yields the equivalence between (i) and (ii).

In order to obtain practical conditions, we have to express the inequalities (91) in terms of the functions $\tau, \eta, \psi$, introduced in (73), (74), and (87), concerning both pairs $(\mathbb{E}, h)$ and $\left(\mathbb{E}^{*}, h^{*}\right)$. To distinguish between them, subsequently, we denote by $\tau^{*}, \eta^{*}, \psi^{*}$ those concerning $\mathbb{E}^{*}$.

Lemma 7.2. Given any two spaces $\mathbb{E}=\operatorname{ker} L$ and $\mathbb{E}^{*}=\operatorname{ker} L^{*}$ of the class $\mathcal{C}$, and any positive $h<\ell_{L}, h^{*}<\ell_{L^{*}}$, assume that $A+A^{*}>0$. Then, we have

$$
\frac{B^{*}-B}{A^{*}+A}-(C+D)=\tau(h) \frac{\left[-\psi(h) \tau^{*^{\prime}}\left(h^{*}\right)-\eta(h) \tau^{* \prime \prime}\left(h^{*}\right)\right]+\tau^{*}\left(h^{*}\right) K(h)}{-\eta(h)\left[\tau^{* \prime}\left(h^{*}\right) \tau(h)+\tau^{*}\left(h^{*}\right) \tau^{\prime}(h)\right]},
$$

with

$$
K(h):=\tau^{\prime \prime}(h)^{2}-\tau^{\prime}(h)\left[1+\tau^{\prime \prime \prime}(h)\right] .
$$

Proof. Since $A=\tau^{\prime}(h) / \tau(h)$ and $B=\tau^{\prime \prime}(h) / \tau(h)$, and similar relations for $A^{*}, B^{*}$, we have

$$
\begin{aligned}
\frac{B^{*}-B}{A^{*}+A}-(C+D) & =\frac{\tau^{* \prime \prime}\left(h^{*}\right) \tau(h)-\tau^{*}\left(h^{*}\right) \tau^{\prime \prime}(h)}{\tau^{* \prime}\left(h^{*}\right) \tau(h)+\tau^{*}\left(h^{*}\right) \tau^{\prime}(h)}+\frac{\psi(h)}{\eta(h)} \\
& =\frac{\tau(h)\left[-\psi(h) \tau^{* \prime}\left(h^{*}\right)-\eta(h) \tau^{* \prime \prime}\left(h^{*}\right)\right]+\tau^{*}\left(h^{*}\right)\left[\eta(h) \tau^{\prime \prime}(h)-\psi(h) \tau^{\prime}(h)\right]}{-\eta(h)\left[\tau^{* \prime}\left(h^{*}\right) \tau(h)+\tau^{*}\left(h^{*}\right) \tau^{\prime}(h)\right]} .
\end{aligned}
$$

Since

$$
\eta(h)=\tau(h) \tau^{\prime \prime}(h)-\tau^{\prime}(h)^{2}, \quad \psi(h)=\tau(h)\left(\tau^{\prime \prime \prime}(h)+1\right)-\tau^{\prime}(h) \tau^{\prime \prime}(h),
$$

we obtain the claimed equality (96).

Thanks to Lemma 7.2 , we can state:

Proposition 7.3. The property (i) of Theorem 7.1 is satisfied if and only if the following two requirements are simultaneously fulfilled:

(a) for any pair $(\mathbb{E}, h) \in \mathcal{F}, h<\min \left(Z_{\tau^{\prime}}, Z_{\psi}\right)$;

(b) for any two pairs $(\mathbb{E}, h)$ and $\left(\mathbb{E}^{*}, h^{*}\right)$ in $\mathcal{F}$,

$$
-\psi(h) \tau^{* \prime}\left(h^{*}\right)-\eta(h) \tau^{* \prime \prime}\left(h^{*}\right)+\tau^{*}\left(h^{*}\right) K(h)>0 .
$$


Proof. For point (a) see Remark 6.4. When (a) holds true, we know that $\eta(h)<0$, and that $\tau(h)$, $\tau^{\prime}(h), \tau^{*}\left(h^{*}\right)$, and $\tau^{* \prime}\left(h^{*}\right)$ are all positive. In particular, the denominator in (96) is positive. The positivity of $\frac{B^{*}-B}{A^{*}+A}-(C+D)$ is therefore equivalent to (98).

Besides, from Lemma 7.2 it is straightforward to derive the following sufficient positivity condition.

Lemma 7.4. Given any two spaces $\mathbb{E}=\operatorname{ker} L$ and $\mathbb{E}^{*}=\operatorname{ker} L^{*}$ of the class $\mathcal{C}$, and any positive $h, h^{*}$ such that $h<\pi / \mu_{L}, h^{*}<\pi / \mu_{L^{*}}$, we can state that

$$
\text { if } \tau^{* \prime \prime}\left(h^{*}\right) \geqslant 0 \text { and } K(h) \geqslant 0 \text {, then } \frac{B^{*}-B}{A^{*}+A}-(C+D)>0 \text {. }
$$

Proof. Under the requirements $h<\pi / \mu_{L}, h^{*}<\pi / \mu_{L^{*}}$, not only do we know that we know that $\tau(h), \tau^{*}\left(h^{*}\right)$ are positive, and that $\eta(h)<0$, but also that $\psi(h)<0$. Accordingly, the implication (99) follows from (96).

\begin{tabular}{|c|c|c|}
\hline Case & $\tau(x)$ & $K(x)$ \\
\hline 1 & $\frac{a \sinh (b x)-b \sinh (a x)}{a b\left(b^{2}-a^{2}\right)}$ & $\frac{[b(\cosh (b x)+1)(\cosh (a x)-1)-a \sinh (a x) \sinh (b x)]^{2}}{\left(b^{2}-a^{2}\right)^{2}(\cosh (b x)+1)(\cosh (a x)-1)}$ \\
\hline 2 & $\frac{\sinh (a x)-a x}{a^{3}}$ & 0 \\
\hline 3 & $\frac{b \sinh (a x)-a \sin (b x)}{a b\left(a^{2}+b^{2}\right)}$ & $\frac{[b(1+\cos (b x))(1-\cosh (a x))+a \sinh (a x) \sin (b x)]^{2}}{\left(a^{2}+b^{2}\right)^{2}(1+\cos (b x))(1-\cosh (a x))}$ \\
\hline 4 & $\frac{b x-\sin (b x)}{b^{3}}$ & 0 \\
\hline 5 & $\frac{b \sin (a x)-a \sin (b x)}{a b\left(b^{2}-a^{2}\right)}$ & $\frac{[b(1+\cos (b x))(1-\cos (a x))-a \sin (a x) \sin (b x)]^{2}}{\left(b^{2}-a^{2}\right)^{2}(1+\cos (b x))(1-\cos (a x))}$ \\
\hline 6 & $\frac{a x \cosh (a x)-\sinh (a x)}{2 a^{3}}$ & $\frac{(\sinh (a x)-a x)^{2}}{4 a^{2}}$ \\
\hline 7 & $\frac{x^{3}}{3}$ & $\frac{(b x-\sin (b x))^{2}}{4 b^{2}}$ \\
\hline 8 & $\frac{\sin (b x)-b x \cos (b x)}{2 b^{3}}$ & $\frac{(b \sinh (a x)-a \sin (b x))^{2}}{4 a^{2} b^{2}}$ \\
\hline 9 & $\frac{a \cosh (a x) \sin (b x)-b \sinh (a x) \cos (b x)}{2 a b\left(a^{2}+b^{2}\right)}$ & $\frac{10}{2}$ \\
\hline
\end{tabular}

Tableau 2: The functions $\tau$ and $K$, see (73) and (97), respectively.

Proposition 7.5. Let $\mathcal{F}$ be composed of all pairs $(\mathbb{E}, h)$ such that either the space $\mathbb{E}$ belongs to the real cases (i.e., Cases 1, 2, 6, 7) and $h$ is any positive number, or $\mathbb{E}$ belongs to Case 4 and $0<h<\frac{\pi}{b}$. Then, all possible spaces of $C^{3}$ splines obtained by mixing pairs of the family $\mathcal{F}$ are piecewise Chebyshevian spline spaces good for design.

Proof. From Table 2 we can see that any pair $(\mathbb{E}, h)$ in $\mathcal{F}$ satisfies $K(h) \geqslant 0$ for all $h>0$. In all real cases we also have $\tau^{\prime \prime}(x)>0$ for all positive $x$. In Case $4, \tau^{\prime \prime}(x)=\sin (b x) / b$ is positive for $x \in] 0, \pi / b[$. The claim follows from Lemma 7.4, Proposition 7.3 and Theorem 7.1.

Remark 7.6. Piecewise exponential spline spaces (i.e., spaces of parametrically continuous splines with sections taken from any different kernels of linear differential operators all of the same orders, with constant coefficients and only real roots for their characteristic polynomials) were considered in [23] (see special cases in $[20,21,7]$ ). We obtained there both a sufficient and a necessary condition for 


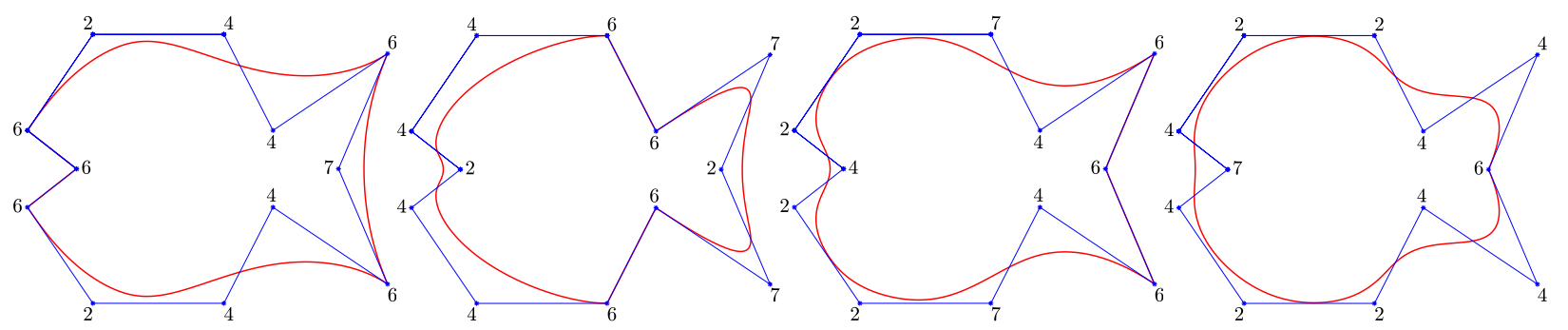

Figure 11: Examples of $C^{3}$ splines with sections taken from Case $2(a=10)$, Case $4(b=3.1415)$, Case $6(a=10)$, and Case 7, as indicated on the control polygons. See Example 7.8.

such spline spaces to be good for design, but no necessary and sufficient condition, except concerning very specific cases. Proposition 7.5 induces that, with order five even characteristic polynomials (72) and with only positive multiplicities, piecewise exponential spline spaces are always good for design, whatever the knots may be. We conjecture that this result extends to any order.

Remark 7.7. Can we similarly use any of the remaining spaces in Table 1 to build parametrically continuous mixed L-splines? From Table 2, we can see that Case 3 behaves differently from the all the other non-real cases: indeed, the corresponding function $K$ is negative on ]0, $+\infty[$, while it is non-negative in all other cases. This is why we first exclude Case 3. Given any pair $(\mathbb{E}, h)$ of the family $\mathcal{F}$ in Proposition 7.5 , and any pair $\left(\mathbb{E}^{*}, h^{*}\right)$, where $\mathbb{E}^{*}$ is taken from Cases $5,8,9$, with $h^{*}<\frac{\pi}{\mu_{L^{*}}}$, Lemma 7.4 ensures that the right inequality in (91) is satisfied. As for the left one, Lemma 7.4 tells us that it will be satisfied too if we additionally require that $\tau^{* \prime \prime}\left(h^{*}\right)>0$. In other words, Proposition 7.5 remains valid when $\mathcal{F}$ is composed of pairs $(\mathbb{E}, h)$ where $\mathbb{E}$ can also be taken from Cases 5, 8, and 9, provided that we then require that $h<Z_{\psi}$ and $h \leqslant Z_{\tau^{\prime \prime}}$. Nevertheless this requirement is only a sufficient condition, and it may unduly limit the possible shape effects.

Below we illustrate Proposition 7.5 as well as Remark 7.7. Beforehand, it should be observed that, for each $k$, the interval $\left[t_{k}, t_{k+1}\right]$ is the central interval of the support $\left[t_{k-2}, t_{k+3}\right]$ of the B-spline $N_{k-2}$. This is why this interval will be represented by the pole $P_{k-2}$. A positive integer $m \leqslant 9$ in front of a pole $P_{k}$ therefore indicates that the section-space $\mathbb{E}_{k+2}$ is taken from Case $m$ in class $\mathcal{C}$. All spline curves presented subsequently preserve symmetry, and this is why the integers are placed symmetrically along the control polygons. In all our illustrations, we deal with equispaced simple knots $t_{k}=k$ for all $k$. As a general principle, our illustrations are mainly intended to show limit shape effects, corresponding to limit values of the parameters involved in the corresponding splines, thus giving the readers an idea about the amplitude of the possible shape effects.

Example 7.8. Let us first illustrate Proposition 7.5. In [10] we observed that, in spite of its additional parameter, Case 1 does not significantly provide more shapes than Case 6 . This is why we limit ourselves to $C^{3}$ splines with sections taken from Cases 2,6, 7, 4. On the other hand, it is interesting to replace the quadric space $\mathcal{P}_{4}$ by another space only if this space provides shapes which are sufficiently different from $\mathbb{E}=\mathcal{P}_{4}$. In this respect, it is not worthwhile using small values of the positive parameter $a$ involved either in $\mathbb{E}^{*}$ (Case 2) or in $\mathbb{E}^{* *}$ (Case 6), for the corresponding curves are closed to quadric curves. By contrast, for big values of the positive parameter $a$, the spaces $\mathbb{E}^{*}$ and $\mathbb{E}^{* *}$ behave as the polynomial spaces $\mathcal{P}_{2}$ and $\mathcal{P}_{0}$, respectively. For instance, for $a=10$, visually speaking there is hardly any difference between splines with all sections taken from Case 2, and quadratic splines. Still the first ones are $C^{3}$ while the others are only $C^{1}$. Similarly for $a=10, C^{3}$ splines with all sections from Case 6 visually coincide with the control polygons. The value $a=10$ is thus taken as the limit value $a=+\infty$ in the few illustrations presented in Fig. 11, while $b=3.1415$ corresponds to the limit value for Case 4 for similar reasons. The reader can easily imagine the 
variations in shape as the couple $(a ; b)$ of parameters goes from $\left(0^{+} ; 0^{+}\right)$(curve coinciding visually with the quadric spline) up to the limit $(10 ; 3.1415)$ used in Fig. 11. The richness of this example is of course increased by changing the parameters independently for the three cases $2,4,6$.

Example 7.9. Here, we consider a pair $\left(\mathbb{E}^{*}, h^{*}\right)$, where $\mathbb{E}^{*}$ comes from case 8 . To ensure (iv) of Theorem 6.3, we first assume that $h^{*}<\pi$. As observed in Remark 7.7, we can include such a pair in the family $\mathcal{F}$ while maintaining the results of Proposition 7.5 provided that $\tau^{* \prime \prime}(1)>0$. From Table 2, we can derive that $2 b \tau^{* \prime \prime}(1)=\sin (b)+b \cos (b)$. The first positive zero $b_{0}$ of $\tau^{* \prime \prime}(1)$ as a function of $b$ is located in ]2.02,2.03[. Unfortunately, bounding above $b$ by $b_{0}$ prevents us to benefit from the limit effects of $C^{3}$ splines with sections taken from $\mathbb{E}^{*}$, obtained when the parameter $b$ approaches $\pi^{-}$. Suppose that we want to mix $\mathbb{E}^{*}$ only with $\mathbb{E}=\mathcal{P}_{4}$. Consider the corresponding double inequality (91). From Lemma 7.4 we know that the right one is always satisfied. Accordingly, in order to always obtain good for design splines, it is necessary and sufficient to ensure that

$$
\frac{B^{*}-6}{A^{*}+3}>-2,
$$

or, as well, that $B^{*}+2 A^{*}>0$, that is,

$$
2 b\left[\tau^{* \prime \prime}(1)+2 \tau^{* \prime}(1)\right]=3 \sin b+b \cos b>0 .
$$

The only zero $b_{0}^{*}$ of the quantity in the left-hand side of the previous inequality as a function of $b$ on ] $0, \pi[$ lies in $] 2.4556,2.4557\left[\right.$. This new upper bound $b_{0}^{*}$ already allows for stronger shape variations than $b_{0}$. The interest of this bound is that it easily permits an interactive selection of the sections where to replace $\mathcal{P}_{4}$ by $\mathbb{E}^{*}$. Oppositely, for a given configuration, it may be possible to go beyond $b_{0}^{*}$. Illustrations are given in Fig. 12. In all four pictures, the red curve corresponds to the value $b=2.4556=b_{0}^{*-}$ for all sections from Case 8 . In the two middle curves, this is an exact bound, since the two sequences $\left(\mathbb{E}, \mathbb{E}^{*}, \mathbb{E}\right),\left(\mathbb{E}^{*}, \mathbb{E}, \mathbb{E}^{*}\right)$ are encountered in the corresponding configurations (see Proof of Theorem 7.1). In the leftmost picture, we never find the sequence $\left(\mathbb{E}^{*}, \mathbb{E}, \mathbb{E}^{*}\right)$. Therefore we do not have to require the left inequality in (7.1) to be valid. However, it is necessary to replace it by the weaker requirement concerning the sequence $\left(\mathbb{E}, \mathbb{E}, \mathbb{E}^{*}\right)$. It can be checked that

$$
\widetilde{\Gamma}\left(\mathbb{E}, \mathbb{E}, \mathbb{E}^{*}\right)>0 \quad \Leftrightarrow \quad \frac{B^{*}-B}{A^{*}+A}>\frac{C^{2}-D^{2}}{-D} .
$$

With $\mathbb{E}=\mathcal{P}_{4}$, the latter condition can be written $B^{*}+3 A^{*}+3>0$, or, equivalently,

$$
\tau^{* \prime \prime}(1)+3 \tau^{* \prime}(1)+3 \tau^{*}(1)>0 .
$$

The resulting upper bound $\widetilde{b}_{0}^{*}$ lies in $] 2.773 ; 2.774[$. In the rightmost picture, in theory we have to additionally take account of the condition $A+A^{*}>0$, but this is satisfied whenever $b<\widetilde{b}_{0}^{*}$. In both configurations, the blue curves correspond to $b=\widetilde{b}_{0}^{*-}=2.773$.

Example 7.10. Let us do the same with $\mathbb{E}^{*}$ taken from Case 9 , thus depending on the two positive parameters $a, b$, and we assume that $b<\pi$. Then,

$$
2 a b\left[\tau^{* \prime \prime}(1)+2 \tau^{* \prime}(1)\right]=(a \cosh a+2 \sinh a) \sin b+b \sinh a \cos b .
$$

Clearly, if $b \leqslant \frac{\pi}{2}$, this quantity is positive for all values of $a$. However, again it is interesting to build splines mixing $\mathcal{P}_{4}$ and $\mathbb{E}^{*}$ only if the shapes permitted by $\mathbb{E}^{*}$ sufficiently differ from the shapes provided by $\mathcal{P}_{4}$. We know that this occurs in particular for "big" values of $a$, all the more efficiently as $b$ is closer to the limit $\pi^{-}$. The problem is that, when $b$ is really close to $\pi^{-}$, for $\tau^{\prime \prime}(1)+2 \tau(1)$ 

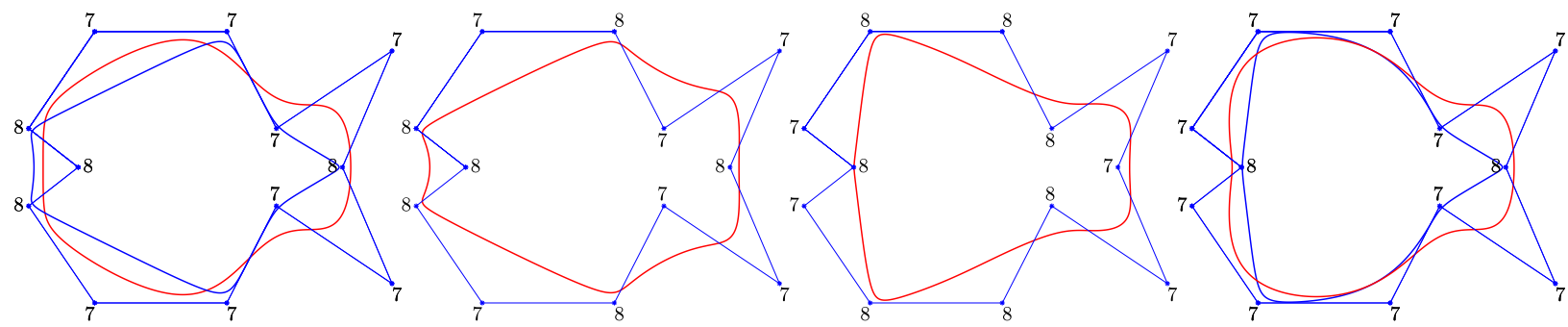

Figure 12: $C^{3}$ spline curves mixing Case 8 and quadrics (Case 7) as indicated on the control polygons.

Blue curves: $b=2.773$ (the tauter ones); red curves: $b=2.4556$. See comments in Example 7.9.

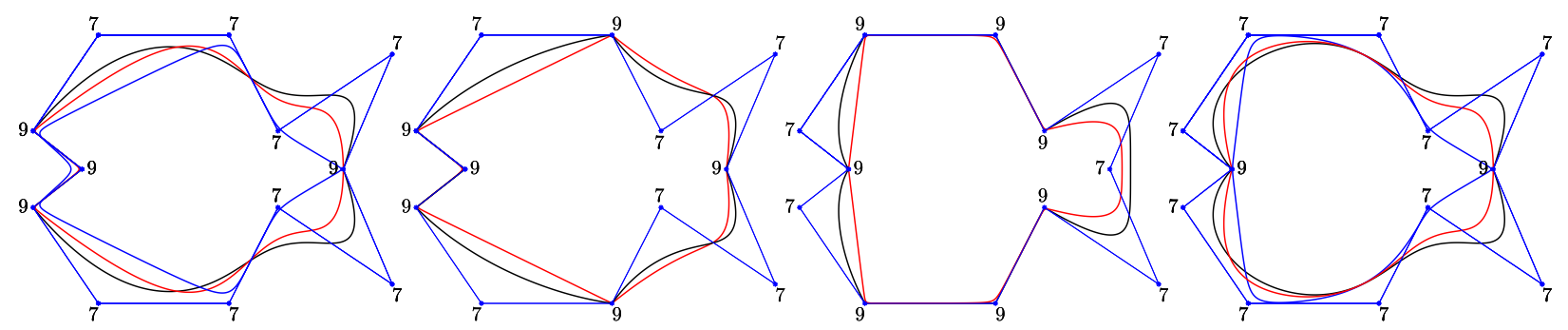

Figure 13: $C^{3}$ spline curves mixing Case 9 and quadrics (Case 7) as indicated on the control polygons. For Case 9, everywhere $b=2.8$. Blue curves: $a=2.82$ (the furthest from the control polygons at the tail); red curves (the closest to the control polygon at the tail): $a=5.876$; black curves: $a=11$. See comments in Example 7.10.

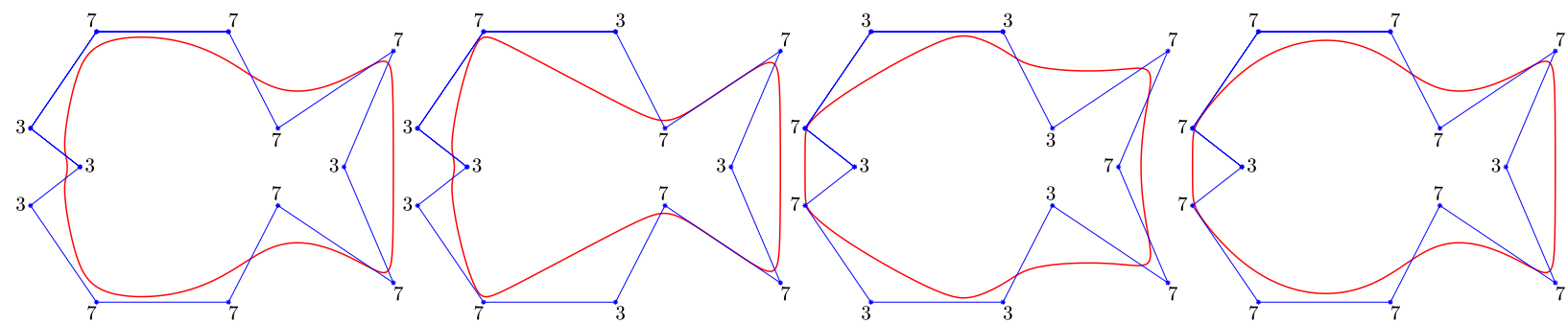

Figure 14: $C^{3}$ spline curves mixing Case 3 (with $b=2.5$ and $a=5.331$ ) and quadrics (Case 7) as indicated on the control polygons. See comments in Example 7.11.

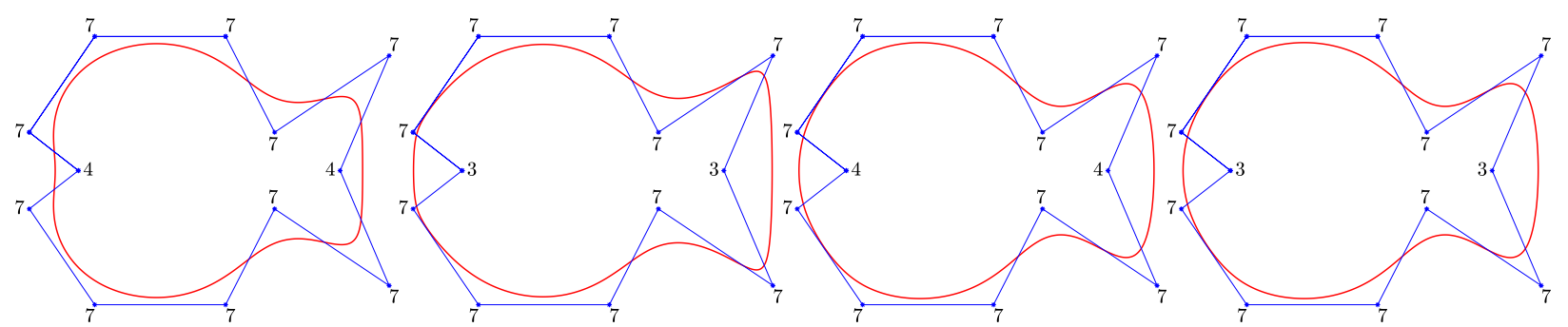

Figure 15: $C^{3}$ spline curves mixing Cases $3 / 4$ and quadrics. From left to right: $b=3.2$ (Case 4); $b=3.2$ and $a=2.3$ (Case 3 ); $b=4.057$ (Case 4$) ; b=4.057$ and $a=0.041$ (Case 3). See comments in Example 7.12. 
to be positive, it will be necessary to take really big values of $a$, which is not reasonable in practice. As a good compromise, take $b=2.8$. This is sufficiently close to $\pi$ to permit significative tension effects, and sufficiently away from $\pi$ to not require unreasonable values for $a$. Indeed, for $b=2.8$, the only zero $a_{0}$ of the right-hand side of (101) as a function of $a$ on $] 0,+\infty[$ lies in $] 5.875 ; 5.8576$ [. In Fig. 13, we have taken the same four configurations as in Fig. 12, simply replacing Case 8 by Case 9. Everywhere the red curves are obtained for $a=a_{0}^{+}=5.8576$. For the same reasons as in Example 7.9, they correspond to exact lower bounds for the two middle curves, but not for the two others. For the leftmost picture, the lower bound $a_{0}^{*}$ resulting from the weaker condition (100) lies in $] 2.819 ; 2.82$ [. Since for $b=2.8, A+A^{*}$ is always positive, this is also the lower bound for the rightmost picture. This bound yields the two limit blue spline curves. Finally in all pictures, the black curves are the limit curves for $a \rightarrow+\infty$, obtained for $a=11$.

Example 7.11. We now want to similarly mix $\mathbb{E}=\mathcal{P}_{4}$ with the space $\mathbb{E}^{*}$ from Case 3 which depends on two positive parameters $a, b$ too, with $b<\pi$. Contrary to Case 8 and 9 , it is now the left inequality in (91) which is satisfied for all values of $a$. Therefore, all spline spaces mixing $\mathbb{E}$ and $\mathbb{E}^{*}$ are good for design if and only if the right inequality in (91) holds. The problem is that this condition does not provide a lower bound for $a$ but an upper one, thus preventing us to benefit from the shape effects obtained when $a \rightarrow+\infty$. Besides, this upper bound is all the smaller as $b$ is closer to $\pi^{-}$. To obtain a reasonably high upper limit, take $b=2.5$. In that case, the upper limit $a_{0}$ is located in ]5.331; 5.332[. The corresponding limit curves are shown in Fig. 14 with $a=a_{0}^{-}=5.331$. Comparison between Figs. 13 and 14 confirms that Cases 9 and 3 produce "opposite" shape effects, as was observed in [10].

Example 7.12. For a complete understanding of $C^{3}$ mixed L-splines, it is worthwhile addressing the following question. Can we get rid of the limit required by (a) of Proposition 7.3 to obtain good for design $C^{3}$ splines mixing a non-real case $\mathbb{E}^{*}$ with $\mathbb{E}=\mathcal{P}_{4}$ ? Here, with $h=h^{*}=1$, and with all non-real cases used in our illustrations so far, can we take $b \geqslant \pi$ ? This is why we now focus on rightmost configuration in Figs. 12-14 (the only one which does not contain three consecutive section-spaces obtained from $\mathbb{E}^{*}$ ). In Case 8 , as well as in Case 9 , it can easily be checked that the positivity condition (100) requires $b<\pi$. Oppositely, in Case 3, this condition is always satisfied for $0<b<2 \pi$. As a matter of fact, the same holds true for Case 4 which is the limit of Case 3 when $a \rightarrow 0^{+}$. In these two cases, assuming that $b \geqslant \pi$, the conditions for the space $\mathbb{S}$ to be good for design come from the rightmost inequality in (91). In Case 4, from (96) and Table 2, this condition is equivalent to

$$
b^{4}\left[\psi^{*}(1)+2 \eta^{*}(1)\right]=b^{2}(\cos (b)+1)+4(\cos (b)-1)<0 .
$$

The good for design upper bound $b_{0}$ is thus located in ]4.0575, 4.0576[. In Case 3, the upper bound $b_{0}^{*}$ for $b$ to make it possible to find positive values of $a$ satisfying the right part of (91) is obtained by solving the corresponding limit inequality (96) when $a \rightarrow 0^{+}$. One can check that $\left.b_{0}^{*} \in\right] 4.0576,4.06[$. Given $b<b_{0}^{*}$, the upper bound $a_{0}^{*}$ for $a$ is all the closer to zero as $b$ is closer to $b_{0}^{*}$. In Fig. 15, we present two curves produced by Case 4 for $b=3.2$ and $b=4.057$, and for these two values of $b$, the limit curves produced by Case 3, with $a=a_{0}^{-}$, that is, $a=2.3$ and $a=0.041$, respectively. No visual difference can be detected between the two curves on the right.

\section{L-splines: local shape effects via connection matrices}

In this section we consider again geometrically continuous polynomial splines as in Subsection 6.2, but this time in the non-cardinal case. A control polygon being given, we would like to analyse the shape effects which can be produced by the insertion of connection matrices, depending on the places where they are inserted, and of the matrices themselves. Out of necessity, we will present 

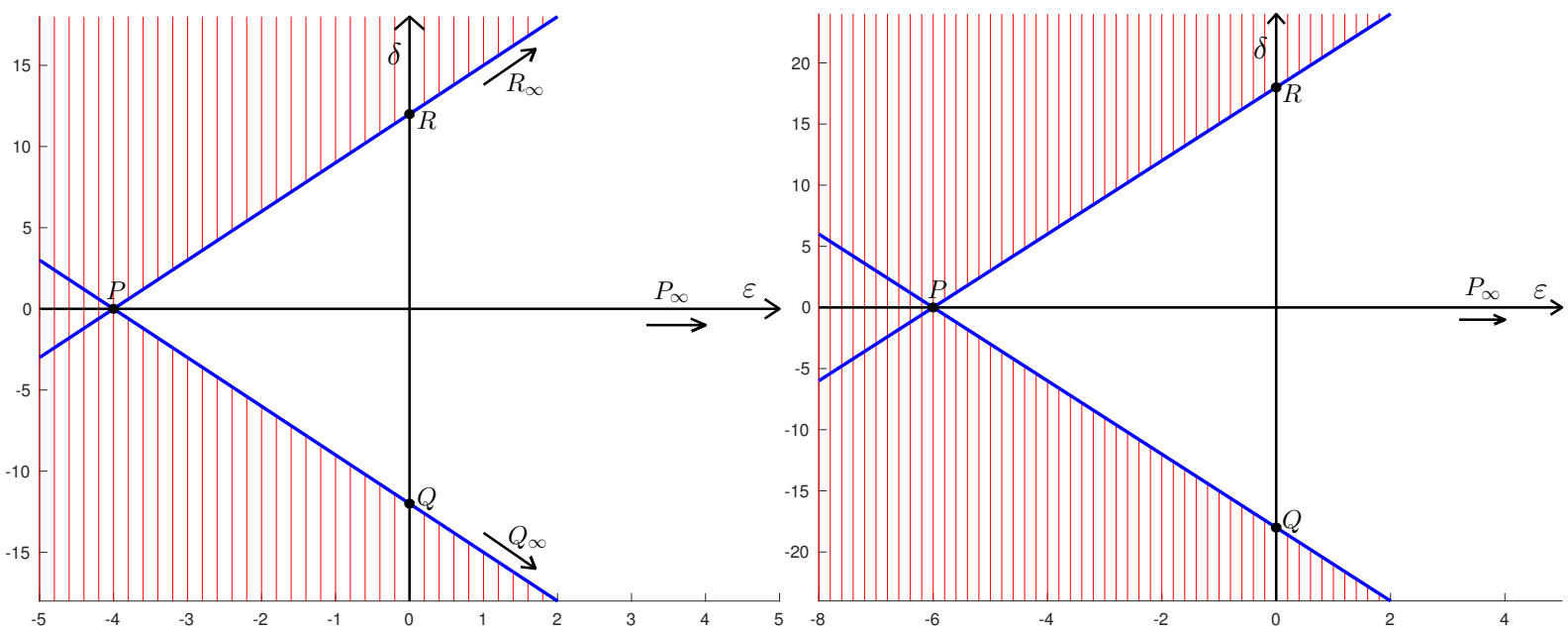

Figure 16: Good for design regions in the plane $(\varepsilon ; \delta)$. Left: Examples 8.1 and Example 8.3, second configuration, with the points $P:=(-4 ; 0), Q:=(0 ;-12), R:=(0 ; 12)$. Right: Example 8.3, first configuration, with the points $P:=(-6 ; 0), Q:=(0 ;-18), R:=(0 ; 18)$

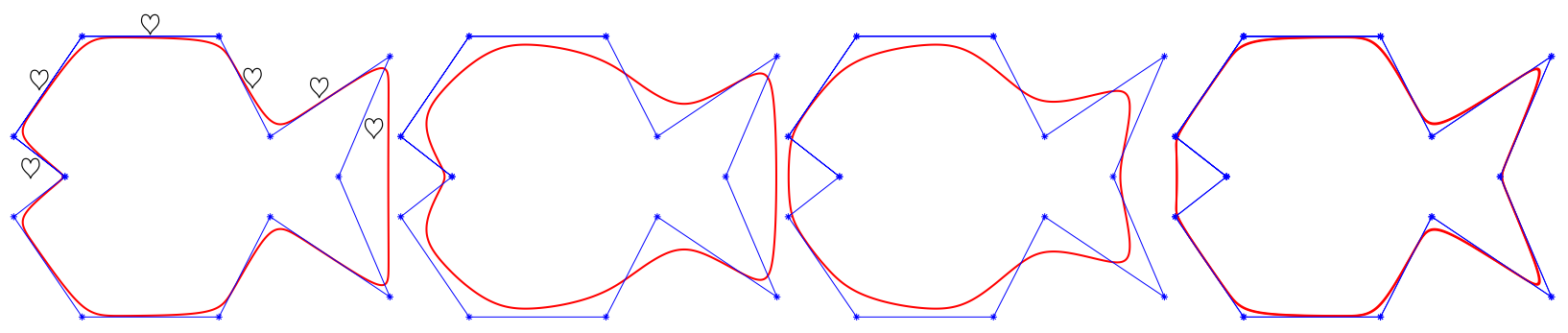

Figure 17: Symmetric $C^{2}, F^{3}$ quartic splines with connection matrix $M_{\odot}$ at all knots indicated by a $\varnothing$ on the leftmost control polygon, and $M_{\diamond}$ at all the symmetric knots, see (102). From left to right, the pair $(\varepsilon ; \delta)$ is equal to $Q_{\infty}:=(75 ;-236) ; Q:=(0 ;-11.99) ; R:=(0 ; 11.99) ; R_{\infty}:=(75 ; 236)$, see left region in Fig.16. See comments in Example 8.1.

very little among all possibilities. The guiding line to select the few examples investigated below follows two principles: limit the number of shape parameters to handle, and preserve the symmetry of the control polygons. Moreover, in each of the examples addressed, we put special emphasis on the limit shapes.

A given knot $t_{k}$ is the middle of the interval $\left[t_{k-3}, t_{k+3}\right]$ obtained as the union of the supports of the two consecutive B-splines $N_{k-3}, N_{k-2}$. This is why $t_{k}$ will be represented by the segment $\left[P_{k-3}, P_{k-2}\right]$ of the control polygon. In all examples, we will consider a given connection matrix $M_{\odot}$, and we will indicate on the leftmost control polygon of a given line of pictures all the knots where the connection matrix is $M_{\odot}$. To preserve symmetry, at the symmetric knot(s), we will implicitly insert the connection matrix $M_{\diamond}$ deduced from $M_{\diamond}$ according to (59). At all other knots, if any, the splines will be assumed to be $C^{3}$.

With simple knots at the integers, all our illustrations will indeed concern polynomial splines that is, no additional parameters come from the section-spaces. Still, without more difficulty the good for design regions in the space of parameters will be determined with all section-spaces taken from one single space in the class $\mathcal{C}$, and with any constant knot spacing $h$. 
Example 8.1. In this example, the connection matrix $M_{\diamond}$ and its "symmetric" matrix $M_{\diamond}$ are given by

$$
M_{\diamond}:=\left[\begin{array}{lll}
1 & 0 & 0 \\
0 & 1 & 0 \\
\delta & \varepsilon & 1
\end{array}\right], \quad M_{\diamond}:=\left[\begin{array}{rcc}
1 & 0 & 0 \\
0 & 1 & 0 \\
-\delta & \varepsilon & 1
\end{array}\right],
$$

where $\delta, \varepsilon$ are any real numbers. We take the connection matrix $M_{\odot}$ everywhere above the symmetry line of the control polygon, and $M_{\diamond}$ everywhere under it. The splines are thus $C^{2}$ everywhere. All $\widetilde{B}_{k}$ 's being equal to $\widetilde{B}:=2 A$, their positivity is ensured if and only if $A>0$, and we assume that this is satisfied. With obvious notations, we have two values for the $\widetilde{D}_{k}$ 's, $\widetilde{\Delta}_{k}$ 's, namely

$$
\widetilde{D}_{\diamond}=\delta+A \varepsilon-D \widetilde{B}=\widetilde{\Delta}_{\diamond}, \quad \widetilde{D}_{\diamond}=-\delta+A \varepsilon-D \widetilde{B}=\widetilde{\Delta}_{\odot}
$$

Finally we have four different values for the $\widetilde{\Gamma}_{k}$ 's, that is, on account of (103),

$$
\begin{aligned}
& \widetilde{\Gamma}_{\odot, \diamond}=\left(\widetilde{D}_{\odot}\right)^{2}-C^{2} \widetilde{B}^{2}, \quad \widetilde{\Gamma}_{\diamond, \diamond}=\left(\widetilde{D}_{\diamond}\right)^{2}-C^{2} \widetilde{B}^{2}, \\
& \widetilde{\Gamma}_{\odot, \diamond}=\widetilde{D}_{\odot} \widetilde{D}_{\diamond}-C^{2} \widetilde{B}^{2}=\widetilde{\Gamma}_{\diamond, \diamond .}
\end{aligned}
$$

The positivity of $\widetilde{D}_{\odot}, \widetilde{D}_{\diamond}, \widetilde{\Gamma}_{\odot, \diamond}, \widetilde{\Gamma}_{\diamond, \odot}$, and $\widetilde{\Gamma}_{\odot, \diamond}$, is obtained if and only if

$$
\widetilde{D}_{\diamond}>C \widetilde{B} \text { and } \widetilde{D}_{\diamond}>C \widetilde{B} .
$$

The spline space $\mathbb{S}$ depends on the two free parameters $\widetilde{D}_{\diamond}, \widetilde{D}_{\diamond}$, both ranging over $] C \widetilde{B},+\infty[=$ ] $2 A C,+\infty[$. We could analyse the variations in shape relative to these two parameters on the "good for design" region $] 2 A C,+\infty[\times] 2 A C,+\infty[$. However, we assume that studying the variations relative to the parameters $\delta, \varepsilon$ will be less surprising to readers, and we therefore translate the good for design conditions (105) into

$$
-A[\varepsilon-2(C+D)]<\delta<A[\varepsilon-2(C+D)],
$$

which implies in particular that $\varepsilon-2(C+D)$ must be positive.

Let us illustrate these conditions in the polynomial case with $t_{k}=k$ for all $k$. According to (106), the corresponding "good for design" region $\mathcal{D}$ of the plane $(\varepsilon ; \delta)$ can be described by the double inequation $-3(\varepsilon+4)<\delta<3(\varepsilon+4)$. It is represented in Fig. 16, left. In Fig. 17 we show the limit curves when $\varepsilon \rightarrow+\infty$ on the two boundary lines $\delta=-2.99(\varepsilon+4)$ (point $Q_{\infty}$ ) and $\delta=2.99(\varepsilon+4)$ (point $R_{\infty}$ ), visually obtained for $\varepsilon=75$. It is interesting to see what happens for $\varepsilon=0$, in which case the parameter $\delta$ ranges over the interval ] - 12,12[ (points close to the boundary points $Q$ and $R$ shown in Fig. 16, left, for which we keep the same notations). We give no illustrations on $\delta=0$, for this corresponds to the $C^{2}$ splines of the cardinal case already shown in Fig. 2. In particular, we can therefore see the shape evolutions along the boundary. The pictures in Fig. 16 clearly point out the symmetry between positive and negative values of the parameter $\delta$.

Example 8.2. In this example, as well as in the following ones, we assume that all connection matrices are the identity matrix, except at a few places. In particular, in all situations considered, we have at least two consecutive knots where the splines are $C^{3}$. Accordingly, the two conditions $A>0, C+D<0$, are necessary for the spline space to be good for design. This is why we directly assume them to be satisfied, so that we only have to consider the good for design conditions involving at least one matrix different from the identity.

In our first example, we replace the identity matrix (denoted here by $I$ ) by the matrix $M_{\odot}$ defined in (102) at one knot above the symmetry line, and therefore by the matrix $M_{\diamond}$ in (102) at the symmetric knot. As previously, the quantities $\widetilde{B}_{k}$ are all equal to $\widetilde{B}=2 A$, and, depending on 


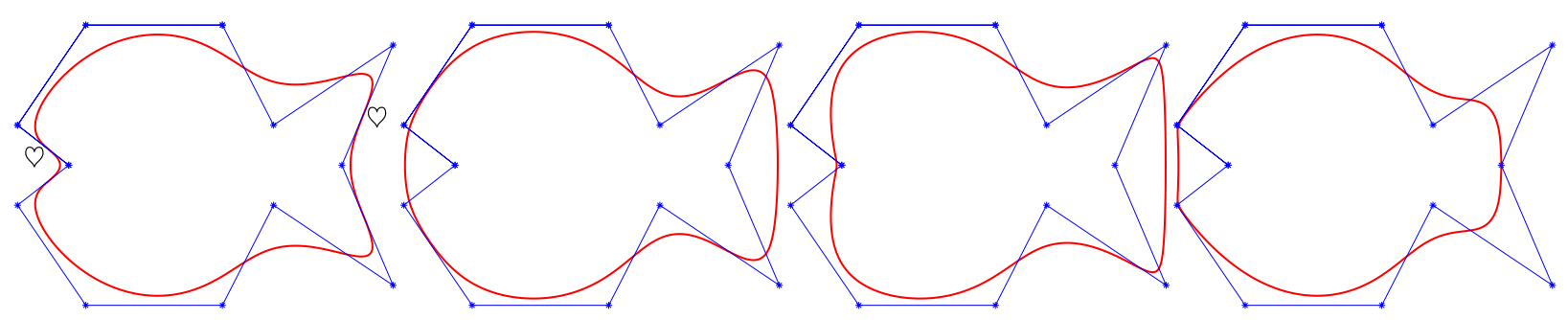

Figure 18: Example 8.2, with the matrix $M_{\odot}$ in (102), and good for design region in the plane $(\varepsilon ; \delta)$ shown in Fig. 16, left. From left to right, parameters at $P_{\infty}=(75 ; 0), P=(-3.99 ; 0)$, $Q=(0 ;-11.99)$, and $R_{\infty}=(75 ; 236)$.

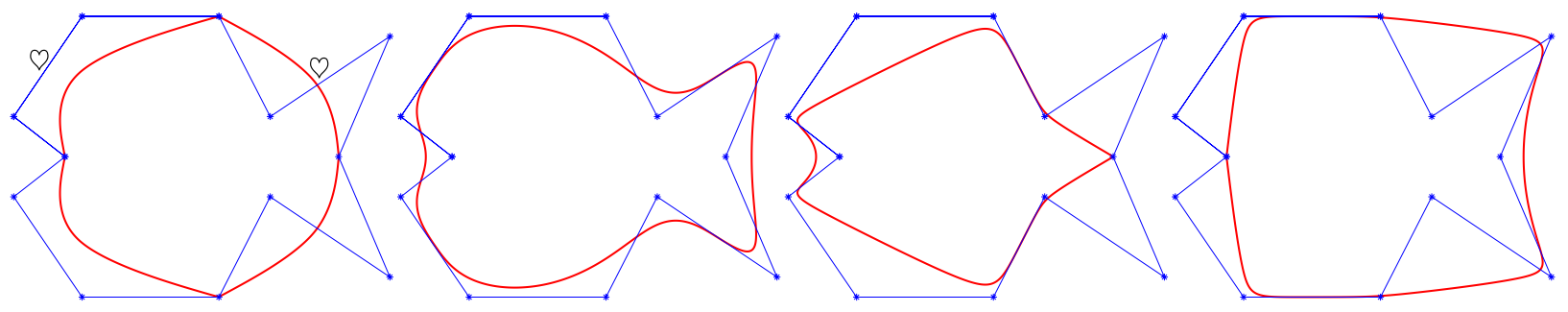

Figure 19: Example 8.2, with good for design region in the plane $(\varepsilon ; \delta)$ shown in Fig. 16, right. From left to right, parameters at $P=(-5.99 ; 0), P_{\infty}=(75 ; 0), Q=(0 ;-17.99)$, and $R=(0 ; 17.99)$.

the knot considered, we have three possibilities for the $\widetilde{D}_{k}$ 's, denoted as $\widetilde{D}=-D \widetilde{B}=-2 A D$, and $\widetilde{D}_{\diamond}, \widetilde{D}_{\diamond}$ as in (103). To find the good for design conditions, we have to consider two different cases.

- The matrices $M_{\diamond}$ and $M_{\diamond}$ are not consecutive. Then, we find somewhere the sequence of consecutive connection matrices $\left(I, M_{\odot}, I\right)$. The corresponding quantities $\widetilde{\Gamma}_{k}$ of which we have to consider the positivity are (with obvious notations)

$$
\widetilde{\Gamma}_{I, \odot}=\widetilde{D} \widetilde{D}_{\diamond}-C^{2} \widetilde{B}^{2}, \quad \widetilde{\Gamma}_{\odot, I}=\widetilde{D} \widetilde{D}_{\odot}-C^{2} \widetilde{B}^{2}
$$

In the present situation, all good for design conditions reduce to

$$
\widetilde{D}_{\odot}>\frac{C^{2} \widetilde{B}^{2}}{\widetilde{D}}, \quad \widetilde{D}_{\diamond}>\frac{C^{2} \widetilde{B}^{2}}{\widetilde{D}}
$$

that is

$$
-A\left[\varepsilon+\frac{2\left(D^{2}-C^{2}\right)}{-D}\right]<\delta<A\left[\varepsilon+\frac{2\left(D^{2}-C^{2}\right)}{-D}\right] .
$$

For polynomial splines with simple knots at the integers, condition $(107)$ gives $-3(\varepsilon+6)<\delta<$ $3(\varepsilon+6)$. This example is illustrated in Fig. 19 with parameters $(\varepsilon ; \delta)$ close to the points $P, Q, R$ indicated in Fig. 16, right, located on the boundary of the good for design region. We simply slightly move these points to be inside the good for design region. Nevertheless we keep the same notation for the modified points. With the additional point $P_{\infty}$, we can see the variations in shape along the axes and also along the boundary.

- The matrices $M_{\diamond}$ and $M_{\diamond}$ are consecutive. This means that we have to consider the positivity conditions resulting form the sequence of connection matrices $\left(M_{\diamond}, M_{\circlearrowleft}, I\right)$, which amounts to ensuring the positivity of $\widetilde{\Gamma}_{\odot, I}$ as in the previous case, along with that of the quantity $\widetilde{\Gamma}_{\diamond, \odot}$ expressed 


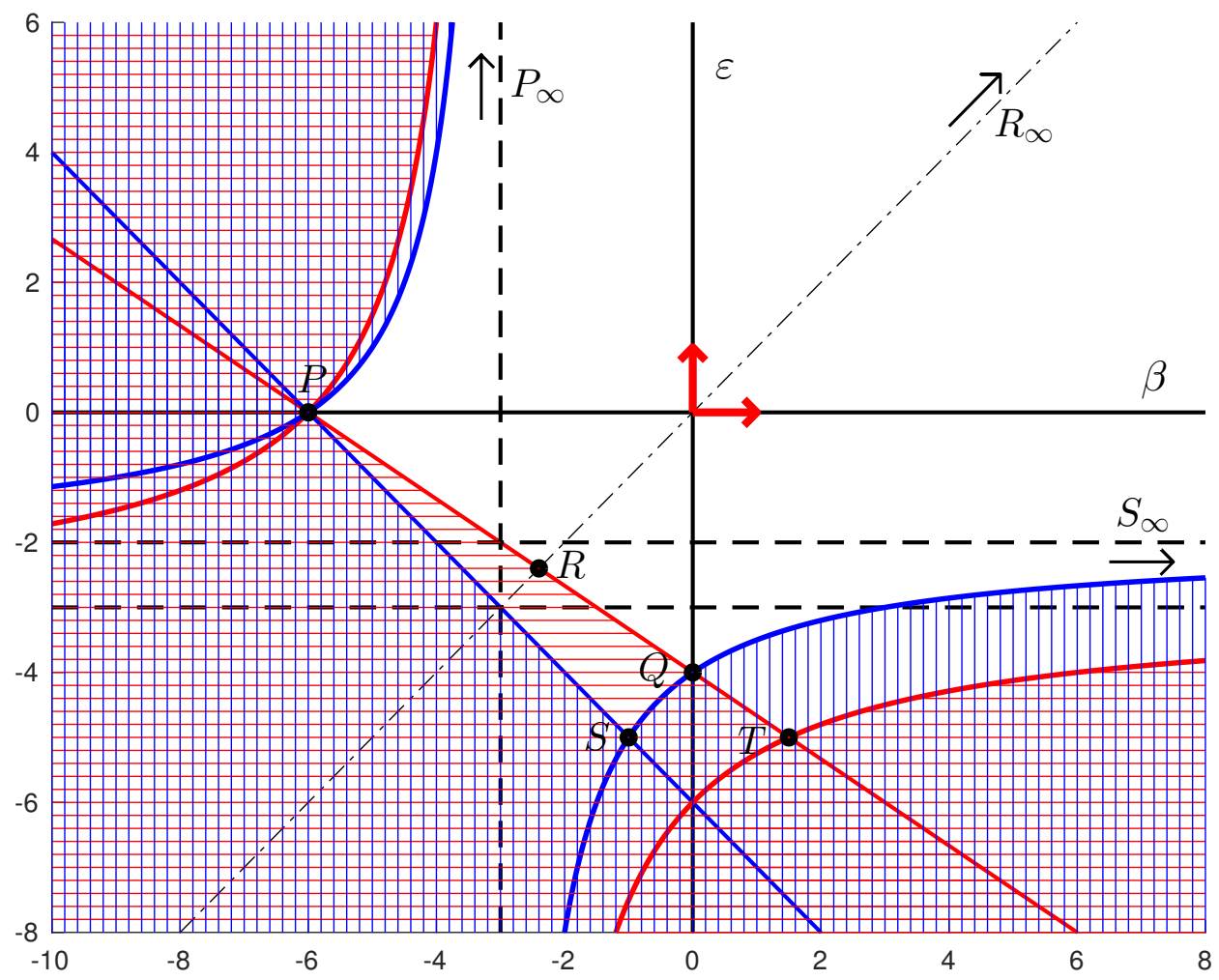

Figure 20: Good for design regions in Example 8.3, depending on the way we place the matrices $M_{\odot}$ and $M_{\diamond}$ given in (110) at the mouth and tail. Red hyperbola: $(\beta+3)(\varepsilon+3)+9=0$; red line: $2 \beta+3 \varepsilon+12=0$, with on it the points $P:=(-6 ; 0), R:=(-2.4 ;-2.4), Q:=(0 ;-4)$, and $T:=(1.5 ;-5)$. Blue hyperbola: $(\beta+3)(\varepsilon+2)+6=0$; blue line: $\beta+\varepsilon+6=0$, with on it the point $S:=(-1 ;-5)$.

in (104). The good for design conditions are thus

$$
\widetilde{D}_{\odot}>\frac{C^{2} \widetilde{B}^{2}}{\widetilde{D}}, \quad \widetilde{D}_{\diamond}>C \widetilde{B} .
$$

Note that the left condition in (109) is a special case of (64). In our illustrations, we have taken twice the matrix $M_{\diamond}$ above the symmetry line, which means that we encounter the two sequences $\left(M_{\diamond}, M_{\odot}, I\right)$ and $\left(M_{\odot}, M_{\diamond}, I\right)$. The good for design conditions are therefore the cumulation of (109) and of the similar inequalities obtained after exchanging $M_{\odot}$ and $M_{\diamond}$. Since $C+D<0$, it is sufficient to keep the right inequality in (107) and its analogue, which gives the same good for design conditions (105) — or (106) — as in Example 8.1.

For polynomial splines, the good for design region is described by $-3(\varepsilon+4)<\delta<3(\varepsilon+4)$, see Fig. 16, left. Illustrations are presented in Fig. 18, for "limit" points of the parameters in the plane $(\varepsilon ; \delta)$. For instance, here too the point $R_{\infty}=(75 ; 236)$, already used in Example 8.1, corresponds to a visual limit along the upper part of the boundary.

Example 8.3. The situation is similar to the previous one, but with different matrices, again corresponding to each other under symmetry, namely

$$
M_{\triangleright}:=\left[\begin{array}{ccc}
1 & 0 & 0 \\
\beta & 1 & 0 \\
0 & \varepsilon & 1
\end{array}\right], \quad M_{\diamond}=\left[\begin{array}{rrr}
1 & 0 & 0 \\
\beta & 1 & 0 \\
\beta \varepsilon & \varepsilon & 1
\end{array}\right],
$$


for which we will see that the good for design region in the plane $(\beta ; \varepsilon)$ is of a different nature. We take the opportunity of this example to emphasise the importance of the order in which we encounter the successive matrices at the possibly different places. In order not to multiply the illustrations we focus on two consecutive matrices different from the identity both around the mouth and the tail. With obvious notations again, we have

$$
\widetilde{B}_{\circlearrowleft}=\widetilde{B}_{\diamond}=\beta+2 A, \quad \widetilde{D}_{\odot}=\varepsilon A-D \widetilde{B}_{\odot}=\widetilde{\Delta}_{\diamond}, \quad \widetilde{D}_{\diamond}=\beta \varepsilon+\widetilde{D}_{\odot}=\widetilde{\Delta}_{\odot}
$$

We first have to require that $\widetilde{B}_{\odot}$ be positive. Assuming that this is true, as usual the conditions ensuring all good for design conditions will follow from the positivity conditions of the specific $\widetilde{\Gamma}_{k}$ encountered, among the following ones:

$$
\begin{aligned}
& \widetilde{\Gamma}_{\odot, \diamond}=\left(\widetilde{D}_{\odot}\right)^{2}-C^{2}\left(\widetilde{B}_{\odot}\right)^{2}, \quad \widetilde{\Gamma}_{\diamond, \diamond}=\left(\widetilde{D}_{\diamond}\right)^{2}-C^{2}\left(\widetilde{B}_{\odot}\right)^{2}, \\
& \widetilde{\Gamma}_{\odot, I}=\widetilde{\Gamma}_{I, \diamond}=\widetilde{D} \widetilde{D}_{\odot}-C^{2} \widetilde{B} \widetilde{B}_{\odot}, \quad \widetilde{\Gamma}_{I, \diamond}=\widetilde{\Gamma}_{\diamond, I}=\widetilde{D} \widetilde{D}_{\diamond}-C^{2} \widetilde{B} \widetilde{B}_{\odot},
\end{aligned}
$$

- In the first situation (see the leftmost control polygons in Figs. 21-24) the positivity conditions concern only $\widetilde{\Gamma}_{\odot, \diamond}$ and $\widetilde{\Gamma}_{\diamond, I}$, that is, $\widetilde{D}_{\odot}>C \widetilde{B}_{\odot}$ and $\widetilde{D}_{\diamond}>C^{2} \widetilde{B} \widetilde{B}_{\odot} / \widetilde{D}$. They can respectively be written as follows

$$
\varepsilon A-(C+D) \beta-2 A(C+D)>0, \quad(\beta+A)\left(\varepsilon+\frac{D^{2}-C^{2}}{-D}\right)+\frac{A\left(D^{2}-C^{2}\right)}{-D}>0
$$

In the plane $(\beta ; \varepsilon)$, the good for design region is thus a region containing the origin which is limited by the two branches of an hyperbola, and by the line $\varepsilon A-(C+D) \beta-2 A(C+D)=0$. Observe that the condition $\widetilde{B}_{\odot}>0$ is automatically ensured by the requirements $(112)$.

In the polynomial spline case, the good for design region is defined by the two inequations

$$
3 \varepsilon+2 \beta+12>0, \quad(\beta+3)(\varepsilon+3)+9>0 .
$$

It is shown in Fig. 20, where the complementary of this good for design region in the plane $(\beta ; \varepsilon)$ is shaded with (red) horizontal hatching.

- Oppositely, in the second situation (middle-left control polygons in all Figs. 21-24), the positivity

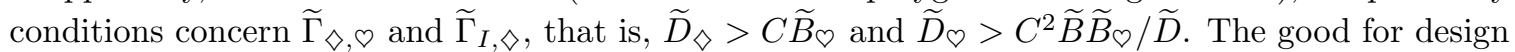
region is defined by the two inequations obtained by adding the quantity $\beta \varepsilon$ to the left-hand side of the first inequation in (112) and subtracting it to the left-hand side of the second inequation in (112).This gives

$$
(\beta+A)(\varepsilon-(C+D))-A(C+D)>0, \quad \varepsilon A+b \frac{D^{2}-C^{2}}{-D}+\frac{2 A\left(D^{2}-C^{2}\right)}{-D}>0,
$$

In the polynomial spline case, we obtain

$$
(\beta+3)(\varepsilon+2)+6>0, \quad \varepsilon+\beta+6>0 .
$$

In the plane $(\beta ; \varepsilon)$ the complementary region is shaded with (blue) vertical hatching.

- In the third and fourth situation (the two control polygons on the right in Figs. 21 - 24) we have to take into account the four quantities, which means the two inequations in (112) along with the two in (113). The good for design region is obtained by intersecting the two regions previously obtained and it can be represented by

$$
\varepsilon A-(C+D) \beta-2 A(C+D)>0, \quad(\beta+A)(\varepsilon-(C+D))-A(C+D)>0
$$




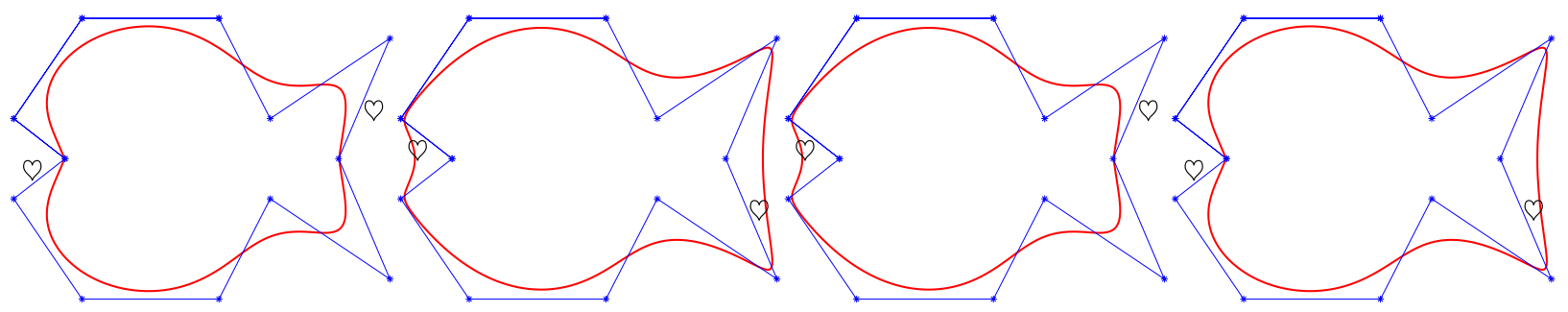

Figure 21: Example 8.3: quadric splines depending on the knots indicated by a $\varnothing$, where the connection matrix $M_{\odot}$ in (110) is inserted, with the connection matrix $M_{\diamond}$ in (110) at the symmetric knots. Here, in the plane $(\beta ; \varepsilon)$, parameters at $P_{\infty}:=(-3 ; 75)$ (see Fig. 20).

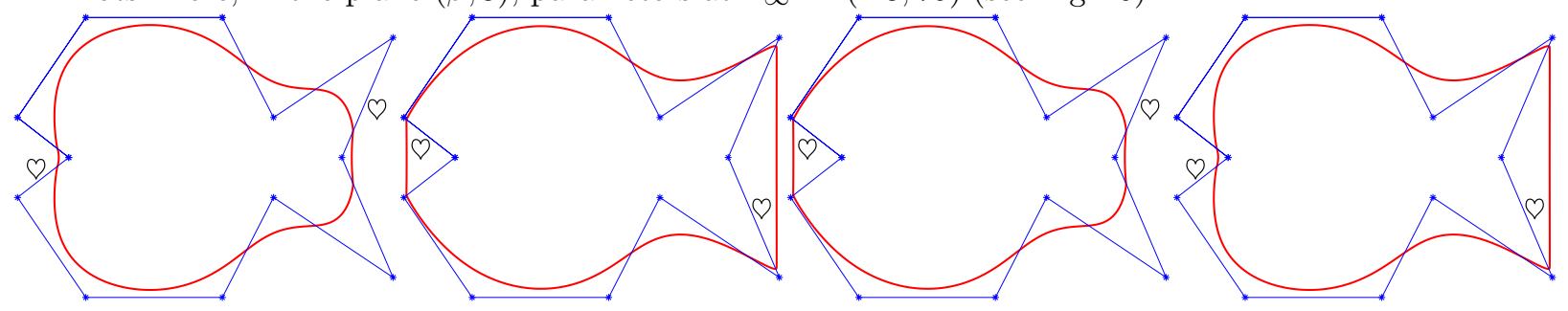

Figure 22: Same as in Fig. 21, with now parameters at $S_{\infty}:=(75 ;-2)$, see Fig. 20.

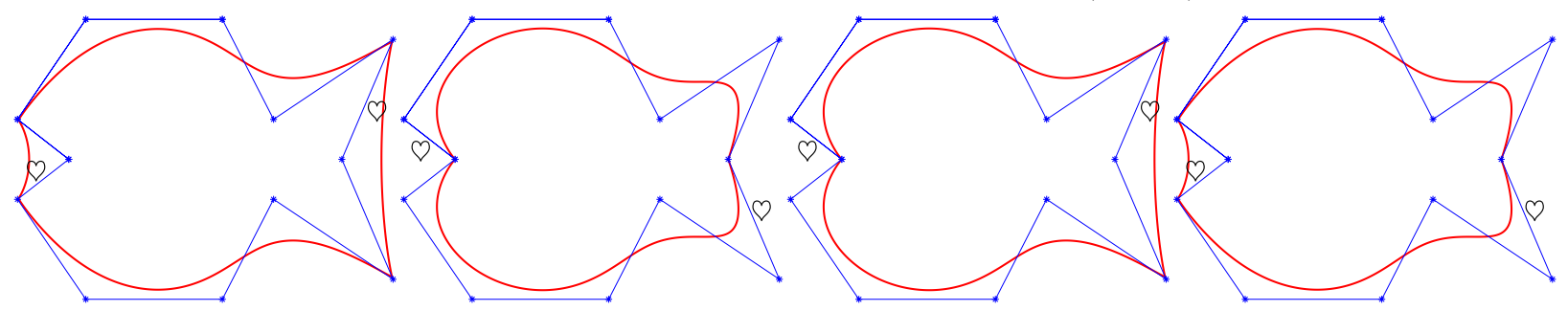

Figure 23: Same as in Fig. 21, with now parameters at $R_{\infty}:=(500 ; 500)$, see Fig. 20.

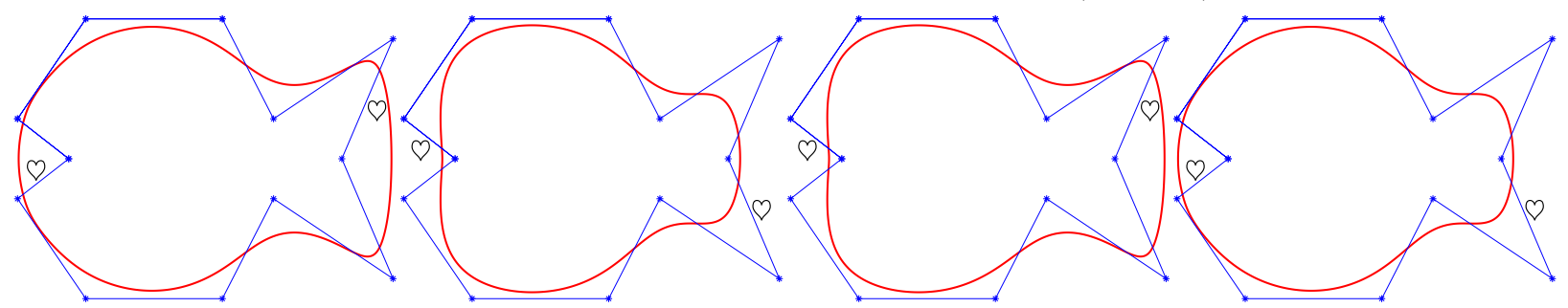

Figure 24: Same as in Fig. 21, with now parameters at $R:=(-2.39 ;-2.39)$, see Fig. 20. 


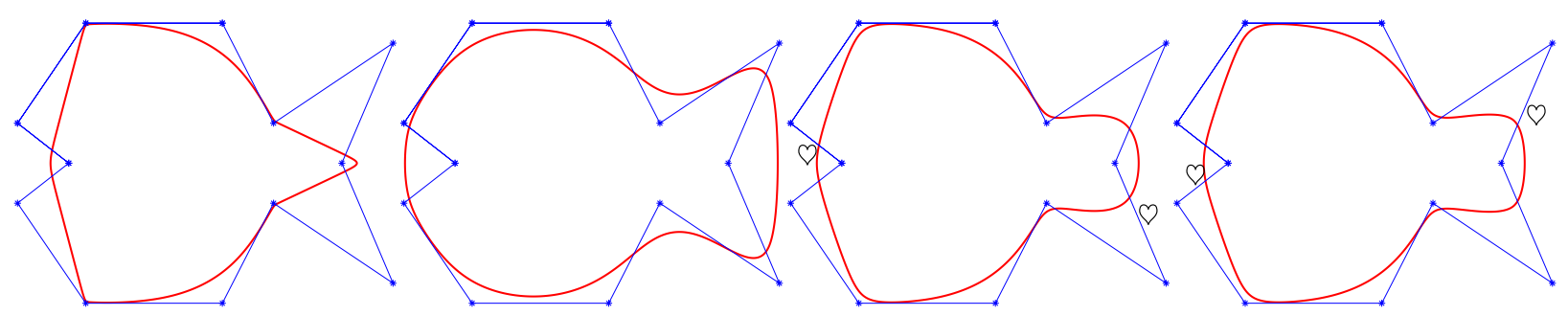

Figure 25: Example 8.3. In the plane $(\beta ; \varepsilon)$, from left to right, parameters at $P:=(-5.99 ; 0)$ and $Q:=$ $(0 ;-3.99)$ (for which $\left.M_{\odot}=M_{\diamond}\right)$, and at the two points $S=(-1 ;-4.99)$ (outside the blue vertically hatched region) and $T=(1.5 ;-4.99)$ (outside the red horizontally hatched region). See Fig. ??

that is, for polynomial splines,

$$
3 \varepsilon+2 \beta+12>0, \quad(\beta+3)(\varepsilon+2)+6>0 .
$$

- To illustrate these results, we first select four points of the good for design region corresponding to $(114)$ in the plane $(\beta ; \varepsilon)$, namely, three points "at infinity": $P_{\infty}$ on the vertical asymptote, $S_{\infty}$ on the horizontal asymptote, $R_{\infty}$ on the diagonal, and finally, a point close to the intersection point $R=(-2.4 ;-2.4)$ of the diagonal and of the line point $3 \varepsilon+2 \beta+12=0$ that we also denote by $R$. At the two points $P:=(-6 ; 0)$ and $Q:=(0 ;-4)$, the two matrices $M_{\odot}$ and $M_{\diamond}$ are equal, see Fig. 25, left (and also the second curve in Fig. 18), which completes the variations along the boundary segment $[P, Q]$. Finally we also show the curves corresponding respectively to (112) and (113) "at" the points $T$ (first configuration) and $S$ (second configuration).

Example 8.4. Our last example will be the simplest case where $M_{\circlearrowleft}=M_{\diamond}$ is the connection matrix $M$ defined in (75) that we already used in the symmetric cardinal case. It is worthwhile considering this case for comparison with the cardinal situation. As usual an heart in front of a segment of the control polygon indicates the presence of the matrix $M$ at the corresponding knot and implicitly at the symmetric segment. As in Example 8.2 we consider two subcases:

- The knots where we insert $M$ are consecutive: then, the good for design conditions are the same as in the cardinal case, that is

$$
\beta+2 A>0, \quad \varepsilon-2(C+D)>0 .
$$

This is illustrated in Fig. 26 for polynomial splines, under the conditions $\beta+6>0$ and $\varepsilon+4>0$.

- The knots where we insert $M$ are not consecutive. The arguments already developed show that the good for design region is characterised by

$$
\beta+2 A>0, \quad \varepsilon+\frac{2\left(D^{2}-C^{2}\right)}{-D}>0 .
$$

that is, in our illustrations in Fig. 27, under the conditions $\beta+6>0$ and $\varepsilon+6>0$. Here we show the shape changes along the diagonal and along the boundary $\varepsilon=-6$, which corresponds to the most significant variations.

\section{Geometrically continuous Piecewise Müntz splines}

So far in our illustrations, symmetry properties were inherent in all section-spaces. Nevertheless, such properties are necessary neither to produce interesting shape effects nor to guarantee symmetry 


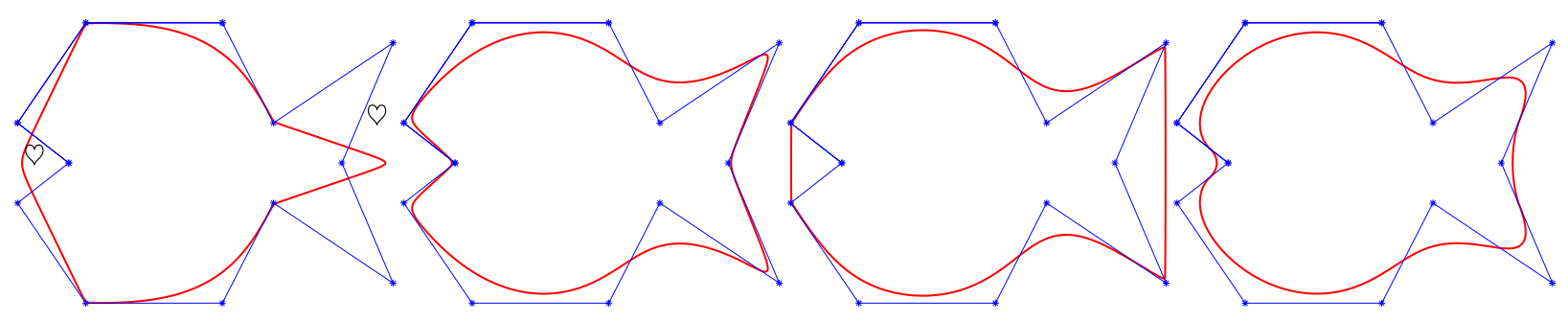

Figure 26: Example 8.4. Good for design region: $(\beta ; \varepsilon) \in]-6,+\infty[\times]-4,+\infty[$. From left to right, $(\beta, \varepsilon)=(-5.99 ;-3.99) ;(-5.99 ; 10000) ;(100 ;-3.99) ;(15 ; 15)$. Ex4TL
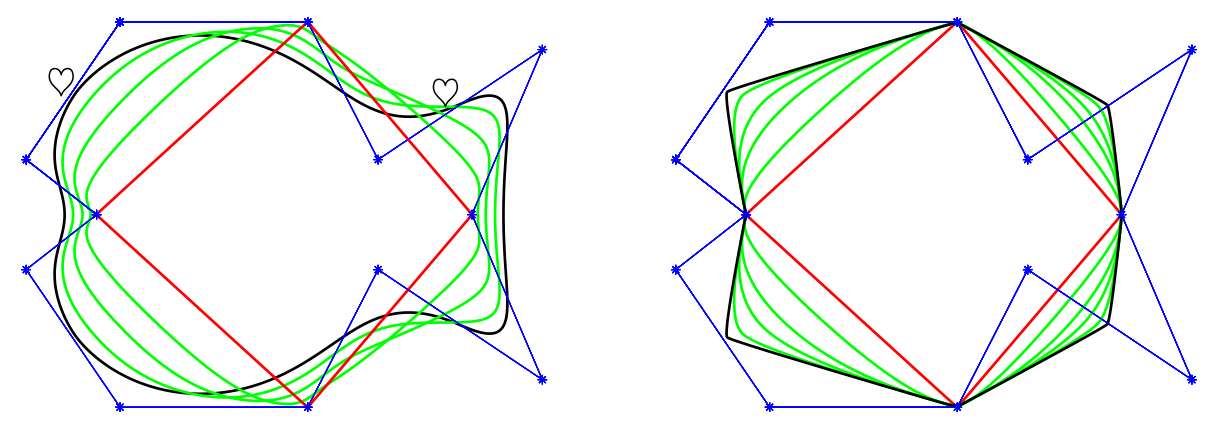

Figure 27: Example 8.4. Good for design region: $(\beta ; \varepsilon) \in]-6,+\infty[\times]-6,+\infty[$. Left : $\varepsilon=\beta=-$ 5.99 (central red curve) ; $-5 ;-4 ;-2 ; 5$ (black limit curve). Right: $\varepsilon=-5.99, \beta=-5.99$ (central red curve) $;-5 ;-3: 0 ; 10 ; 75$ (black limit curve).

preservation by the spline curves. This is the first point that we want to illustrate in the present short section, via a class of geometrically continuous piecewise Müntz spaces which already proved to provide interesting shape effects $[27,30, ?, 2,1,3]$. Our second concern is to provide a first insight into the already mentioned possibility of using quantities involved in the necessary and sufficient conditions for a piecewise Chebyshevian spline space to be good for design as shape parameters rather than the entries of the connection matrices.

Assume that the bi-infinite sequence of simple knots $t_{k}, k \in \mathbb{Z}$, is given, along with the biinfinite sequence $\mathbb{E}_{k}, k \in \mathbb{Z}$, of EC-section-spaces good for design in their intervals. As explained in Subsection ?? we know that the good for design spline space $\mathbb{S}$ can be completely determined, for instance, by the choice of two free bi-infinite sequences of positive numbers $\widetilde{D}_{k}, \widetilde{\Delta}_{k}$, and a third one, $\widetilde{\Gamma}_{k}, k \in \mathbb{Z}$, meeting the requirement (67). In order to reduce the number of parameters, we first assume that we are dealing with $C^{1}$ and $F^{3}$ splines as we did in all illustrations (unit diagonals). It is also rather natural to consider the special case where we require that $\widetilde{D}_{k}=\widetilde{\Delta}_{k}$ for all $k$. This occurs if and only if

$$
\widetilde{D}_{k}=\frac{\widetilde{B}_{k} \widetilde{E}_{k}}{2} \text { for all } k \in \mathbb{Z} \text {. }
$$

Conversely, if we select any positive parameters $\widetilde{B}_{k}, \widetilde{E}_{k}, k \in \mathbb{Z}$, and then define the bi-infinite sequence $\widetilde{D}_{k}, k \in \mathbb{Z}$, as in (115), how to make sure that the corresponding spline space $\mathbb{S}$ will be good for design? The answer is given in the lemma below.

Lemma 9.1. Assume that all matrices have unit diagonals and that (115) holds. Then, the splines space $\mathbb{S}$ is good for design if and only if

$$
\widetilde{E}_{k} \widetilde{E}_{k+1}>4 C_{k}^{+} C_{k+1}^{-} \quad \text { for all } k \in \mathbb{Z} .
$$


Proof. Under our assumptions, we have $\widetilde{\Delta}_{k}=\widetilde{D}_{k}$ for all $k$. Accordingly, for each integer $k$

$$
\widetilde{\Gamma}_{k}=\frac{\widetilde{B}_{k} \widetilde{E}_{k}}{2} \frac{\widetilde{B}_{k+1} \widetilde{E}_{k+1}}{2}-C_{k}^{+} C_{k+1}^{-} \widetilde{B}_{k} \widetilde{B}_{k+1}=\widetilde{B}_{k} \widetilde{B}_{k+1}\left(\frac{\widetilde{E}_{k} \widetilde{E}_{k+1}}{4}-C_{k}^{+} C_{k+1}^{-}\right), \quad k \in \mathbb{Z} .
$$

Whence the claimed result.

Note that the situation examined in Lemma 9.1 has already been encountered twice, first in the symmetric cardinal case (Section ??), second in the non-cardinal case in Example 8.4.

Let us apply the previous result to a class of geometrically continuous piecewise Müntz spline spaces that we define below. From now on, we assume that $t_{k}=k$ for all $k$. To define the sectionspaces, we consider a given bi-infinite sequence of positive numbers $p_{k}, k \in \mathbb{Z}$. Two situations are then to be considered:

- If $p_{k}=p_{k+1}$, then $\mathbb{E}_{k}$ is the restriction to $\left[t_{k}, t_{k+1}\right]=[k, k+1]$ of the polynomial space $\mathcal{P}_{4}$, and in $D \mathbb{E}_{k}$, as usual we use the Bernstein-like basis

$$
V_{i}^{k}(x)=V_{i}\left(x-t_{k}\right), i=0, \ldots, 3,
$$

where

$$
V_{0}(x)=(1-x)^{3}, \quad V_{1}(x)=x(1-x)^{2}, \quad V_{2}(x)=x^{2}(1-x), \quad V_{3}(x)=x^{3}, \quad x \in[0,1] .
$$

- If $p_{k} \neq p_{k+1}$, then, up to the affine change of variable $\vartheta_{k}:\left[t_{k}, t_{k+1}\right] \rightarrow\left[\min \left(p_{k}, p_{k+1}\right), \max \left(p_{k}, p_{k+1}\right)\right]$ such that $\vartheta_{k}\left(t_{i}\right)=p_{i}$ for $i=k, k+1$ - that is, $\vartheta_{k}(x):=p_{k}+\left(p_{k+1}-p_{k}\right)(x-k)$ - the space $\mathbb{E}_{k}$ is spanned by the the four functions $1, x^{\ell+1}, x^{\ell+2}, x^{\ell+3}, x^{\ell+4}$, where $\ell$ is a given positive integer. This is indeed an EC-space good for design on $\left[t_{k}, t_{k+1}\right]$, see $[25,27]$. In that case, the Bernstein-like basis $(28)$ in $D \mathbb{E}_{k}$ is given by

$$
\begin{aligned}
V_{0}^{k}(x) & =\left(\frac{\vartheta_{k}(x)}{p_{k}}\right)^{\ell}\left(\frac{p_{k+1}-\vartheta_{k}(x)}{p_{k+1}-p_{k}}\right)^{3}, \\
V_{1}^{k}(x) & =\left(\frac{\vartheta_{k}(x)}{p_{k}}\right)^{\ell}\left(\frac{p_{k+1}-\vartheta_{k}(x)}{p_{k+1}-p_{k}}\right)^{2}\left(\frac{\vartheta_{k}(x)-p_{k}}{p_{k+1}-p_{k}}\right), \\
V_{2}^{k}(x) & =\left(\frac{\vartheta_{k}(x)}{p_{k+1}}\right)^{\ell}\left(\frac{p_{k+1}-\vartheta_{k}(x)}{p_{k+1}-p_{k}}\right)\left(\frac{\vartheta_{k}(x)-p_{k}}{p_{k+1}-p_{k}}\right)^{2}, \\
V_{3}^{k}(x) & =\left(\frac{\vartheta_{k}(x)}{p_{k+1}}\right)^{\ell}\left(\frac{\vartheta_{k}(x)-p_{k}}{p_{k+1}-p_{k}}\right)^{3} .
\end{aligned}
$$

Accordingly, in both cases, at each knot $t_{k}$, the numbers introduced in (28) are as follows:

$$
\begin{aligned}
& A_{k}^{-}=\frac{\ell\left(p_{k}-p_{k-1}\right)}{p_{k}}+3, \quad B_{k}^{-}=\frac{\ell(\ell-1)\left(p_{k}-p_{k-1}\right)^{2}}{p_{k}^{2}}+6 \frac{\ell\left(p_{k}-p_{k-1}\right)}{p_{k}}+6, \\
& C_{k}^{-}=2\left(\frac{p_{k}}{p_{k-1}}\right)^{\ell}, \quad D_{k}^{-}=-2 \frac{\ell\left(p_{k}-p_{k-1}\right)}{p_{k}}-4, \\
& A_{k}^{+}=-\frac{\ell\left(p_{k+1}-p_{k}\right)}{p_{k}}+3, \quad B_{k}^{+}=\frac{\ell(\ell-1)\left(p_{k+1}-p_{k}\right)^{2}}{p_{k}^{2}}-6 \frac{\ell\left(p_{k+1}-p_{k}\right)}{p_{k}}+6, \\
& C_{k}^{+}=2\left(\frac{p_{k}}{p_{k+1}}\right)^{\ell}, \quad D_{k}^{+}=2 \frac{\ell\left(p_{k+1}-p_{k}\right)}{p_{k}}-4 .
\end{aligned}
$$


In particular, independently of the parameters $\ell$, and of the $p_{k}$ 's, and of the we thus have

$$
C_{k}^{+} C_{k+1}^{-}=4 \text { for all } k \in \mathbb{Z} \text {. }
$$

Thanks to Lemma 9.1, we can thus state:

Proposition 9.2. The space of geometrically continuous piecewise Müntz spline defined above is good for design if and only if

$$
\widetilde{E}_{k} \widetilde{E}_{k+1}>16 \text { for all } k \in \mathbb{Z} \text {. }
$$

In this situation, the shape parameters are the positive numbers $\widetilde{B}_{k}, \widetilde{E}_{k}$, the ratios $p_{k+1} / p_{k}$, $k \in \mathbb{Z}$, which are not equal to one (see $[27,30, ?]$ ), along with the positive integer $\ell$. In theory, without more difficult we could even allow this integer to depend on the intervals. However, to permit a useful handling of the shape effects we rather have to reduce the number of parameters. This is why here, not only do we keep the same $\ell$ everywhere, but we even assume that the numbers $\widetilde{B}_{k}, \widetilde{E}_{k}$, are independent of $k$. We can therefore drop the indices. Then, the B-spline-like $M_{j}$ with support $[j, j+4]$ in the space $D \mathbb{S}$ can be taken as

$$
M_{j}(x)=\left\{\begin{array}{rr}
\left(p_{j+1}\right)^{\ell} V_{3}^{j}(x) \quad \text { if } x \in[j, j+1], \\
\left(p_{j+1}\right)^{\ell} V_{0}^{j+1}(x)+\left(p_{j+1}\right)^{\ell} \widehat{B} V_{1}^{j+1}(x)+\left(p_{j+2}\right)^{\ell} \frac{1}{2} \widehat{B} \widehat{E} V_{2}^{j+1}(x)+\left(p_{j+2}\right)^{\ell} \widehat{E} V_{3}^{j+1}(x) \\
\left(p_{j+2}\right)^{\ell} \widehat{E} V_{0}^{j+2}(x)+\left(p_{j+2}\right)^{\ell} \frac{1}{2} \widehat{B} \widehat{E} V_{1}^{j+2}(x)+\left(p_{j+3}\right)^{\ell} \widehat{B} V_{2}^{j+2}(x)+\left(p_{j+3}\right)^{\ell} V_{3}^{j+2}(x)
\end{array},\right.
$$

where, with notations similar to the cardinal case, $\widehat{B}:=\widetilde{B}$, and $\widehat{E}:=\widetilde{E} / 2$. Moreover, according to (121), we must require that $\widehat{E}>2$. We would like to draw the reader's attention to the fact that the resulting connection matrices are not the same at all knots, since their entries are obtained via (70) and (120). To illustrate such splines, we replace quadric section-spaces by Müntz spaces only at very few places. In other words, almost all positive numbers $p_{k}$ are equal to one. In each figure, a circle on a segment of the control polygon indicates that $p_{k}=p \neq 1$ at the knot represented by this segment. In practice we use the two values $p=10$ and $p=100$. To additionally reduce the number of parameters, we assume that $\widehat{E}=\frac{3}{2} \widehat{B}-5$ as in Figs. 7 and 8. Accordingly, for each integer $k$ such that the two consecutive sections $\mathbb{E}_{k-1}, \mathbb{E}_{k}$ are quadric spaces - that is, whenever $p_{k-1}=p_{k}=p_{k+1}$-, splines in $\mathbb{S}$ are $G^{3}$ at the knot $t_{k}$. They are only $F^{3}$ at the remaining knots. Observe that symmetry preservation is obtained by allocating the same value for $p_{k}$ at symmetric knots. In all our examples, we take $\ell=3$, though it would also be interesting to vary the value of $\ell$. In Fig. 28 the values used for $\widehat{B}$ are exactly the same as those in Fig. 8 for comparison. Finally, in Fig. 29 we limit ourselves to the two values $\widehat{B}=10$ and $\widehat{B}=100$. Moving the two circles all around the control polygon gives a good understanding of the shape effects which can be obtained. On account of how the section-spaces are defined, it should be observed that the number of sectionspaces which are not obtained by restriction of $\mathcal{P}_{4}$ is either two (circles placed consecutively: first and last line in Fig. 29) or four (all other situations, including Fig. 28). 

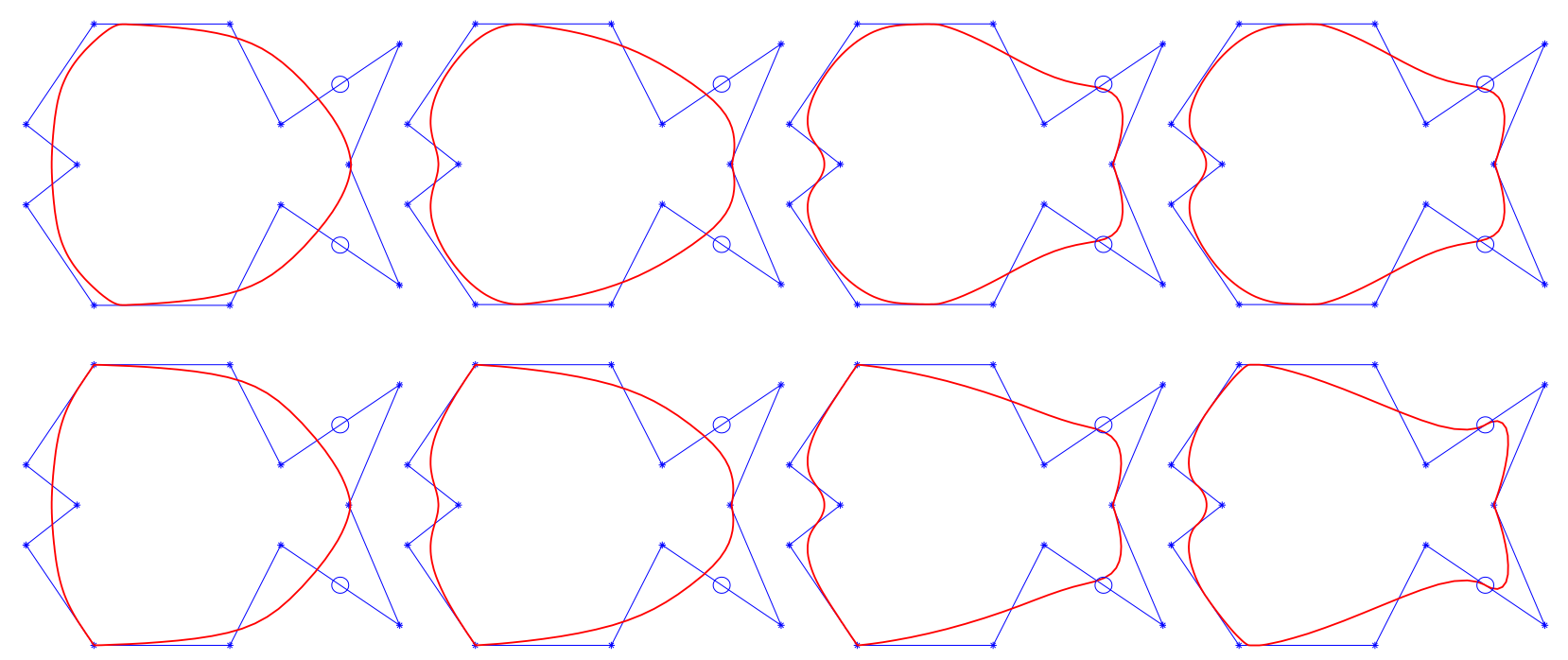

Figure 28: Examples of $C^{1}, F^{3}$ piecewise Müntz splines with $p_{k}=1$ everywhere except "at" the knots indicated with a circle where $p_{k}=10$ (up) or $p_{k}=100$ (down), and with $\ell=3$. From left to right $\widehat{B}:=4.67 ; 6 ; 10 ; 100$, for comparison with Fig. ??.

\section{Concluding comments}

This work was mainly intended to enlighten the following points.

1- Necessary and sufficient conditions permit to take better advantage of the shape effects provided both by the section-spaces and the connection matrices. Moreover, these conditions naturally lead to new types of shape parameters.

2- In dimension five even more than in dimension four, the infinite number of possibilities makes it compulsory to restrict the number of parameters to very few. Even under such restrictions, we obtain an impressive variety of shape variations. The examples developed in this manuscript can help to select an appropriate easy-to-handle framework in view of a given expected improvement in the spline curves produced. As an instance, the class of parametrically continuous mixed Lsplines considered here is especially rich due to the five-dimensional context with enables us to mix trigonometric and hyperbolic functions inside given section-spaces.

3- By comparison with the cubic-like context, the proof of Theorem 4.1 gives good intuition of the difficulty to similarly achieve necessary and sufficient conditions in higher dimensions. This suggests that it might be useful to develop a numerical approach to apply the beautiful theoretical characterisation of all good for design splines reminded in Section 2 (Theorem 2.11) in any dimensions.

4- Nevertheless, if higher dimensions are needed, the richness of the present context can be efficiently exploited using splines obtained by (repeated) integration. For instance, through Proposition 7.5 this provides access to examples of splines visually identical to some so-called multi-degree splines (see, e.g., $[46,47]$ ), with more regularity.

\section{References}

[1] R. Ait-Haddou, Dimension elevation in Müntz spaces: a new emergence of the Müntz condition, J. Approx. Theory, 181 (2014), 6-17. 

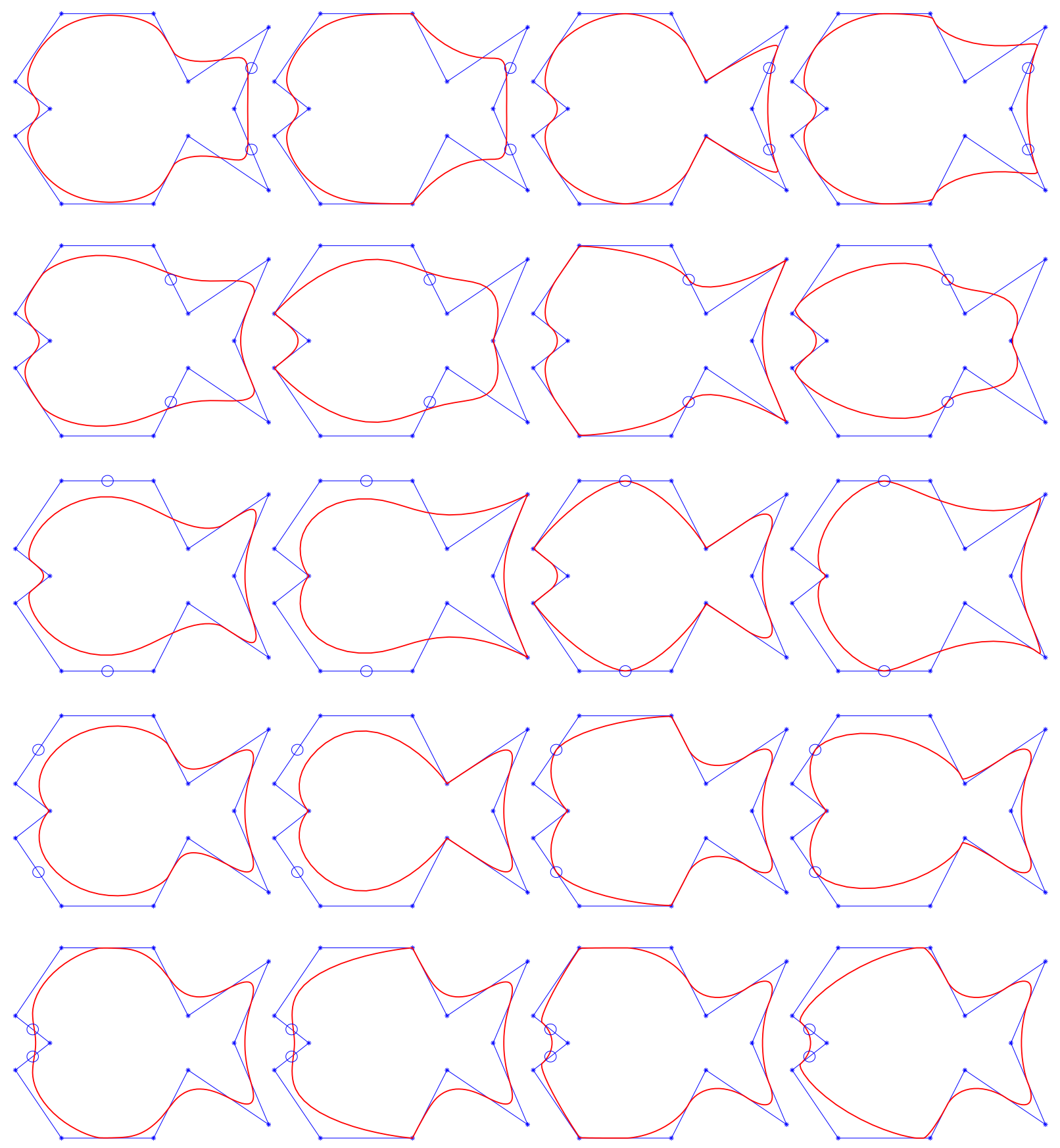

Figure 29: $C^{1}, F^{3}$, piecewise Müntz spaces, with $p_{k}=1$ everywhere except at the knots indicated with circles where $p_{k}=p$. From left to right: $\widehat{B}=10, p=10 ; \widehat{B}=10, p=100 ; \widehat{B}=100, p=10$; $\widehat{B}=100, p=100$. 
[2] R. Ait-Haddou, Y. Sakane, and T. Nomura, Chebyshev blossoming in Müntz spaces: Toward shaping with Young diagrams, J. Comput. Applied Math., 247 (2013), 172-208.

[3] R. Ait-Haddou and M.-L. Mazure, Sparse Pythagorian hodograph curves, Comput. Aided Geom. Design., 55 (2017), 84-103.

[4] Barry P. J., de Boor-Fix dual functionals and algorithms for Tchebycheffian B-splines curves, Constr. Approx., 12 (1996), 385-408.

[5] D. Bister and H. Prautzsch, A New Approach to Tchebycheffian B-Splines in Curves and Surfaces with Applications in CAGD, Vanderbilt University Press, 35-41, 1997.

[6] T. Bosner, Knot insertion algorithms for Chebyshev splines, PhD thesis, Dept of Maths, University of Zagreb, 2006.

[7] T. Bosner and M. Rogina, Non-uniform exponential tension splines, Numer. Algorithms, 46 (2007), 265-294.

[8] T. Bosner and M. Rogina, Quadratic convergence for CCC-Schoenberg operators, Numer. Math., 135 (2017),1253-1287.

[9] Brilleaud M. and M.-L. Mazure, Mixed hyperbolic/trigonometric spaces for design, Comput. Math. Appl., 64 (2012), 2459-2477.

[10] Brilleaud M. and M.-L. Mazure, Design with L-splines, Numer. Algorithms, 65 (2014), 91-124.

[11] Buchwald B. and G. Mühlbach, Construction of B-splines for generalized spline spaces generated from local ECT-systems, J. Comput. Applied Math., 159 (2003), 249-267.

[12] Carnicer J.-M. and J.-M. Peña, Total positivity and optimal bases, in: M. Gasca, C. A. Micchelli (eds.) Total Positivity and its Applications, Math. Appl. 359, Klüwer Academic Publishers, Dordrecht, 133-155, 1996.

[13] J.-M. Carnicer, E. Mainar, and J.-M. Peña, Critical Length for Design Purposes and Extended Chebyshev Spaces, Constr. Approx., 20 (2004), 55-71.

[14] P. Costantini, T. Lyche, and C. Manni, On a class of weak Tchebycheff systems, Numer. Math., 101 (2005), 333-354.

[15] N. Dyn, A. Edelman, and C.A. Micchelli, On locally supported basis functions for the representation of geometrically continuous curves, Analysis, 7 (1987), 313-341.

[16] N. Dyn and C.A. Micchelli, Piecewise polynomial spaces and geometric continuity of curves, Num. Math., 54 (1988), 319-337.

[17] T.N.T. Goodman, Properties of beta-splines, J. Approx. Theory, 44 (1985), 132-153.

[18] Goodman T.N.T., Total Positivity and the Shape of Curves, M. Gasca, C. A. Micchelli (eds.) Total Positivity and its Applications, Math. Appl. 359, Klüwer Academic Publishers, Dordrecht, 157-186, 1996.

[19] Karlin S. J. and W. J. Studden, Tchebycheff Systems: with applications in analysis and statistics, Wiley Interscience, New York, 1966.

[20] P.E. Koch and T. Lyche, Exponential B-splines in tension, in Approximation Theory VI, C. K. Chui, L. L. Schumaker, and J. D. Ward (eds.), Academic Press, NY, 1990, 361-364.

[21] Y. Lü, G. Wang, and X. Yang, Uniform hyperbolic polynomial B-spline curves, Comput. Aided Geom. Design, 19 (2002), 379-393.

[22] T. Lyche and L. L. Schumaker, Total Positivity Properties of LB-splines, in Total Positivity and its Applications, M. Gasca and C. Micchelli (eds.), Klüwer, Dordrecht, 35-46, 1996.

[23] T. Lyche and M.-L. Mazure, Total positivity and the existence of piecewise exponential B-splines, Adv. Comp. Math., 25 (2006), 105-133.

[24] T. Lyche and M.-L. Mazure Piecewise Chebyshevian Multiresolution Analysis, East J. Approx., 17 (4), 419-435, 2012. 
[25] M.-L. Mazure, Vandermonde type determinants and blossoming, Adv. Comp. Math., 8 (1998), 291-315.

[26] M.-L. Mazure, Blossoming: a geometrical approach, Constr. Approx., 15 (1999), 33-68.

[27] M.-L. Mazure, Chebyshev spaces with polynomial blossoms, Adv. Comp. Math., 10 (1999), 219-238.

[28] M.-L. Mazure, Blossoms and optimal bases, Adv. Comp. Math., 20 (2004), 177-203.

[29] M.-L. Mazure, On the equivalence between existence of B-spline bases and existence of blossoms, Constr. Approx., 20 (2004), 603-624.

[30] M.-L. Mazure, Chebyshev splines beyond total positivity, Adv. in Comput. Math., 14 (2001), 129-156.

[31] M.-L. Mazure, Chebyshev spaces and Bernstein bases, Constr. Approx., 22 (2005), 347-363.

[32] M.-L. Mazure, Ready-to-blossom bases in Chebyshev spaces, in: K. Jetter, M. Buhmann, W. Haussmann, R. Schaback, J. Stoeckler (eds.) Topics in Multivariate Approximation and Interpolation, Elsevier, Amsterdam, 109-148, 2006.

[33] M.-L. Mazure Ready-to-blossom bases and the existence of geometrically continuous piecewise Chebyshevian B-splines, CRAS, 347 (2011), 829-834.

[34] M.-L. Mazure, Finding all systems of weight functions associated with a given Extended Chebyshev space, J. Approx. Theory, 163 (2011), 363-376.

[35] M.-L. Mazure, How to build all Chebyshevian spline spaces good for geometric design?, Numer. Math., 119 (2011), 517-556.

[36] M.-L. Mazure, Polynomial splines as examples of Chebyshevian splines, Numer. Algorithms, 60 (2012), 241-262.

[37] M.-L. Mazure, On a new criterion to decide whether a spline space is suitable for design, BIT Num. Math., 52 (2012), 1009-1034.

[38] M.-L. Mazure, Piecewise Chebyshev-Schoenberg operators: shape preservation, approximation and space embedding, J. Approx. Theory, 166 (2013), 106-135.

[39] M.-L. Mazure, Piecewise Chebyshevian splines: Interpolation versus Design, to appear in Numer. Algorithms.

[40] Mühlbach G., ECT-B-splines defined by generalized divided differences, J. Comput. Applied Math., 187 (2006), 96-122.

[41] Mühlbach G. and Y. Tang, Computing ECT-B-splines recursively, Num. Algorithms, 41 (2006), 35-78.

[42] G. Mühlbach, One sided Hermite interpolation by piecewise different generalized polynomials, J. Comput. Applied Math., 196 (2006), 285-298.

[43] Pottmann H., The geometry of Tchebycheffian splines, Comput. Aided Geom. Design, 10 (1993), 181210.

[44] L. Ramshaw, Blossoms are polar forms, Comput. Aided Geom. Design, 6 (1989), 323-358.

[45] Schumaker L. L. Spline Functions, Wiley Interscience, New York, 1981.

[46] T. Sederberg, J. Zheng, X. Song, Knot intervals and multi-degree splines, Comput. Aided Geom. Design, 20 (2003), 455-468.

[47] W. Shen, G. Wang, A basis of multi-degree splines, Comput. Aided Geom. Design, 27 (2010), 23-35. 